\title{
Interplay of ARID1A and EGFR signaling in controlling acinar cell reprogramming in the pancreas
}

\author{
DISSERTATION \\ for the award of the degree \\ "Doctor of Philosophy (Ph.D.)" \\ Division of Mathematics and Natural Sciences \\ of the Georg-August-Universität Göttingen \\ within the doctoral program "Molecular Medicine" \\ of the Georg-August University School of Science (GAUSS)
}

Submitted by

Zhe Zhang

Born in

Baotou, China

Göttingen, December 2020 



\section{Members of the Thesis Committee}

PD Dr. med. Elisabeth Hessmann

Department of Gastroenterology, Gastrointestinal Oncology \& Endocrinology

University Medical Center, Göttingen

Prof. Dr. Bernd Wollnik

Institute of Human Genetics

University Medical Center, Göttingen

Prof. Dr. Argyris Papantonis

Institute of Pathology

University Medical Center, Göttingen

Extended Examination Board Members:

Prof. Dr. Matthias Dobbelstein

Institute of Molecular Oncology

University Medical Center, Göttingen

Dr. Nico Posnien

Department of Developmental Biology

University of Göttingen

Dr. Ufuk Günesdogan

Göttingen Center for Molecular Biosciences

University of Göttingen

Date of submission of thesis: 28.12.2020

Date of the oral examination: 11.02.2021 



\section{Table of Contents}

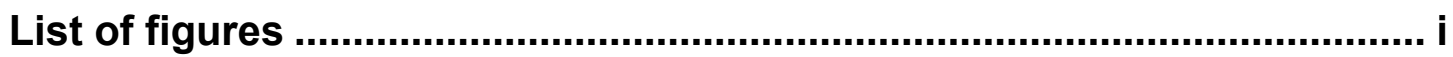

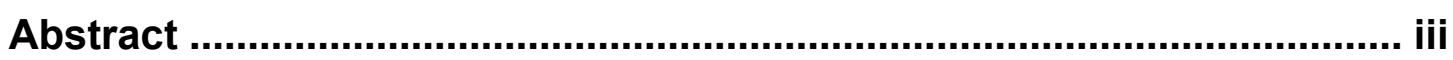

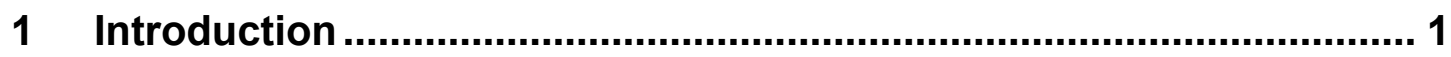

1.1 Precursor lesions of pancreatic ductal adenocarcinoma ..................... 1

1.2 Pancreatic acinar cell reprogramming .................................................. 2

1.3 EGFR signaling .................................................................................

1.3.1 EGFR signaling cascade ............................................................. 3

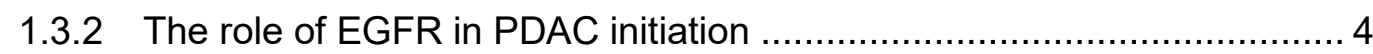

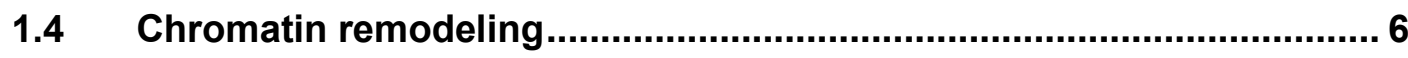

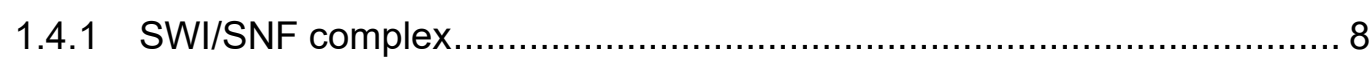

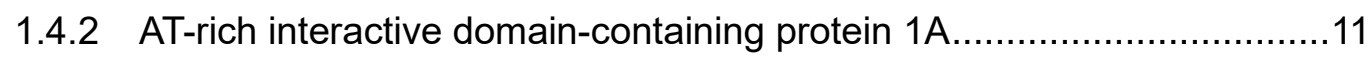

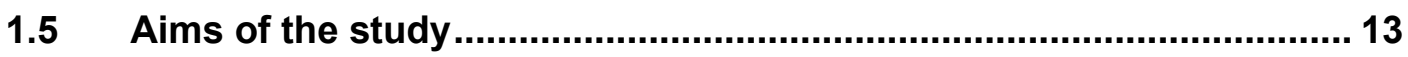

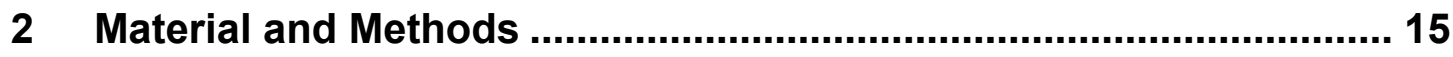

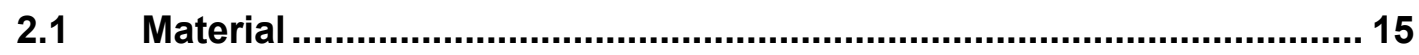

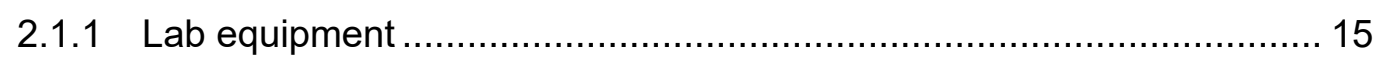

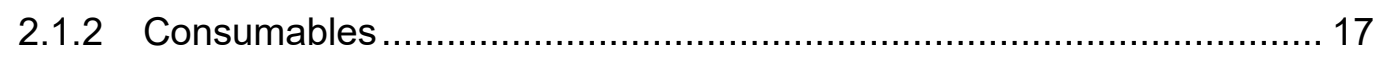

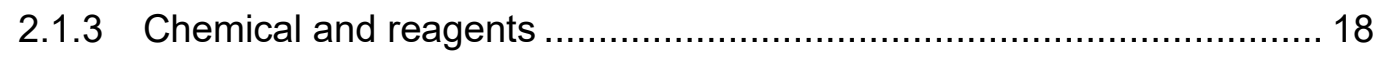

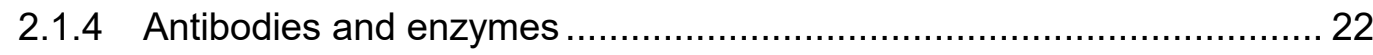

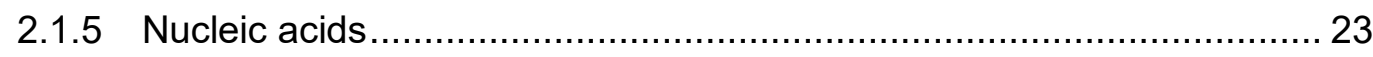

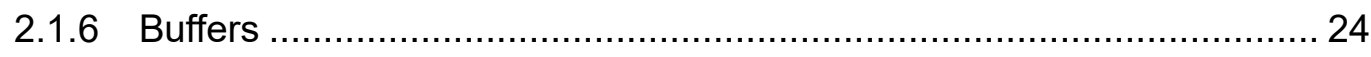

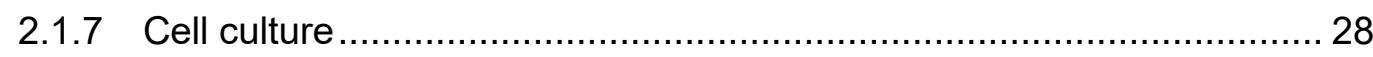

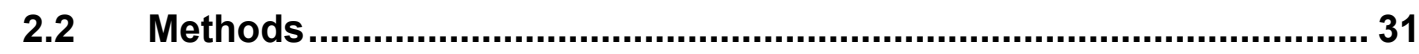

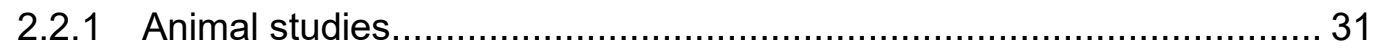

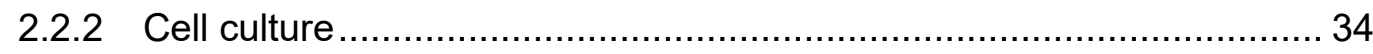

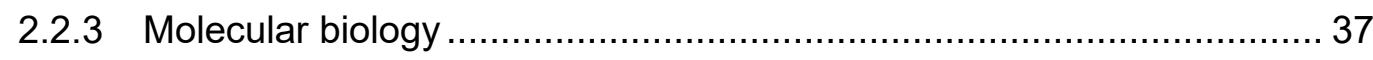

2.2.4 Protein biochemistry .................................................................... 39

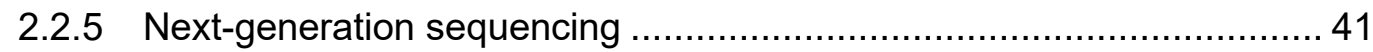

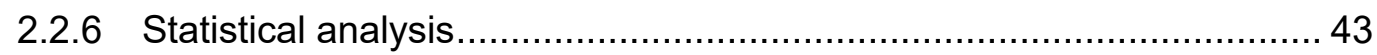

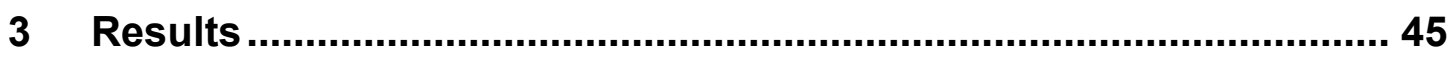

3.1 ARID1A is required to maintain pancreatic acinar cell identity and 
prohibits pre-neoplastic lesion formation 45

3.1.1 Arid1a deficiency causes acinar cell loss and duct dilation..... 45

3.1.2 Arid1a loss caused transcriptional shifts in pancreatic acinar cell identity50

3.2 Arid1a loss increases chromatin accessibility . 53

3.3 Arid1a deficiency cooperates with EGFR signaling in driving acinar cell reprogramming 54

3.3.1 Arid1a loss enhances EGF-induced acinar to ductal transdifferentiation54

3.3.2 Detection of gene signatures driving cellular plasticity upon EGFR signaling activation and Arid1a deficiency.... 59

3.3.3 ARID1A binds transcriptionally active promoters and enhancers 61

\subsection{EGFR signaling activity leads to genome-wide ARID1A dissociation} from the genome 64

3.5 NFATc1 drives ADM formation in the context of Arid1a loss and EGFR signaling by displacing ARID1A from the genome. 67

3.5.1 NFATc1 is required for Arid1a loss-induced acinar reprogramming..... 67

3.5.2 NFATc1 disposes ARID1A from its target genes 71

4 Discussion .75

4.1 The role of ARID1A in modulating pancreatic acinar cell plasticity ... 75

4.2 Arid1a loss alters the epigenomic landscape. 77

4.3 The activity of the residual SWI/SNF complex upon inactivation of individual subunit. 80

4.4 EGFR signaling in pancreatic cancer initiation and progression ........ 81

4.4.1 Arid1a deficiency cooperates with EGFR signaling in driving pancreatic acinar cell programming 81

4.4.2 The role of EGFR signaling in regulating genomic disposition of ARID1A83

4.4.3 EGFR signaling leads to genomic ARID1A dissociation via activating NFATc1 .85

4.5 Concluding remarks 88

5 References. 89

Acknowledgements 113 


\section{List of figures}

Figure1. The progression model of pancreatic epithelial neoplasia $(\operatorname{Pan} \mid \mathrm{N}) \ldots \ldots \ldots \ldots \ldots \ldots . .2$

Figure 2. Conventional diagram of epidermal growth factor receptor (EGFR) signaling pathway

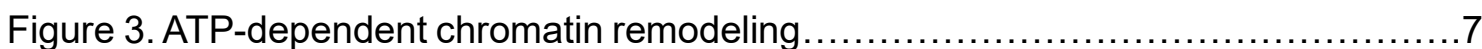

Figure 4. Mammalian SWI/SNF are polymorphic complexes.........................

Figure 5. Arid1a deficiency affects mice pancreas in a dose-dependent manner........46

Figure 6. Arid1a deficiency leads to exocrine insufficiency phenotype of mice...........47

Figure 7. ARID1A is necessary for maintaining pancreatic acinar cell integrity..........48

Figure 8. Arid1a loss induces ductal-lesion formation and inflammation infiltration......49

Figure 9. Conventional diagram of acinar cell isolation and quality assessment of RNAseq 50

Figure 10. Arid1a loss activates signaling pathways promoting acinar cell dedifferentiation

Figure 11. Arid1a deficiency increases chromatin accessibility. 53

Figure 12. EGFR is overexpressed in Arid1a-deficient mice. .54

Figure 13. Arid1a deficiency cooperates with EGFR signaling in driving primary acinar cell reprogramming 56

Figure 14. Arid1a deficiency cooperates with EGFR signaling in driving ADM in the context of oncogenic Kras.

Figure 15. Alterations led by Arid1a-knockdown and EGFR activity in acinar and ductal markers expression .58

Figure 16. Arid1a deficiency and EGFR signaling cooperate for transcriptional activation of gene signatures driving cell plasticity

Figure 17. ARID1A binds active chromatin

Figure 18. ARID1A binds active promoters and enhancers. 63

Figure 19. EGFR signaling activation reduces genome-wide ARID1A occupancy.... 64

Figure 20. EGFR signaling induces ARID1A disassociation from chromatin to nucleoplasm. .65

Figure 21. Tracks of ChIP- and ATAC-seq peaks of Bhlhe40, Lif and Met gene 66.

Figure 22. NFATc1 binds a subset of ARID1A target genes and is trans-activated by 


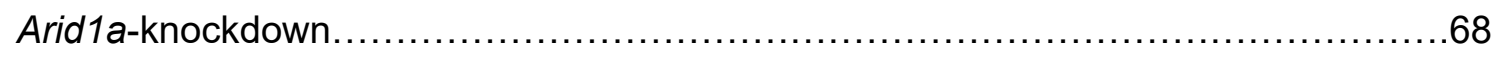

Figure 23. NFATc1 activity promotes ADM formation in the context of Arid1a

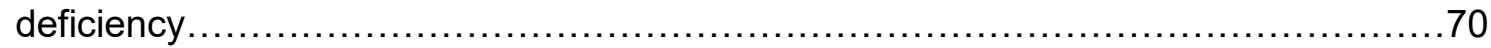

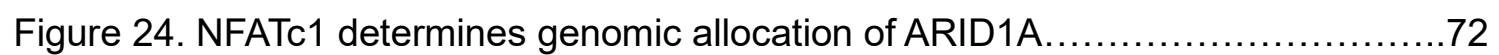

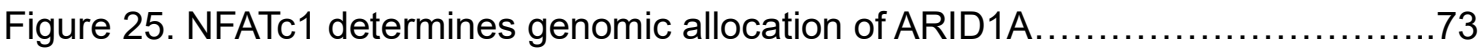

Figure 26. Model of EGFR-ARID1A-axis induced metaplastic transcriptional activation. .87 


\section{Abstract}

Based on a high degree of cellular plasticity and triggered by external (e.g. inflammation) or internal (e.g. oncogenic pressure) signals, pancreatic acinar cells can undergo reprogramming processes resulting in acinar-to-ductal metaplasia (ADM). Functionally, $A D M$ is involved in pancreatic regeneration processes, but also represents the initiating event of pancreatic carcinogenesis. Acinar reprogramming is under coordinated regulation of genetic, epigenetic and environmental factors, among which, SWItch/Sucrose Non-Fermentable (SWI/SNF) -mediated chromatin remodeling plays a pivotal role. The SWI/SNF complex, and particularly its DNA-binding subunit AT-rich interaction domain-containing protein $1 A(A R I D 1 A)$, is crucial for determining cell fate and maintaining terminal cell differentiation. Loss-of-function mutations of $A R I D 1 A$, as recurrently detected in PDAC patients, lead to aberrant chromatin regulation and altered gene transcription, and have been shown to disrupt acinar cell homeostasis and accelerate oncogenic Kras-driven pancreatic carcinogenesis. However, the mechanistic background of ARID1A-dependent maintenance of acinar cell integrity and the interaction of the SWI/SNF member with upstream signaling cues have not been elucidated yet. Herein, we aimed at i) exploring the consequences of ARID1A loss on chromatin regulation during acinar-to-ductal metaplasia, and ii) characterizing the interplay of ARID1A with epidermal growth factor receptor (EGFR) signaling, a known inducer of $A D M$, in determining acinar cell fate.

In order to dissect how EGFR signaling and ARID1A-mediated chromatin regulation interact in acinar reprogramming we combined in vivo and in vitro models with highthroughput sequencing methods. We confirmed that Arid1a deficiency drives ADM in the pancreas which is accompanied by upregulation of EGFR expression. Arid1adeficiency and EGFR signaling cooperate in promoting the ADM phenotype. Furthermore, we showed that the cooperation between EGFR signaling activation and Arid1a deficiency in acinar cell reprogramming was executed through creating a chromatin state permissive ductal gene transcription programs. Moreover, EGFR interferes with ARID1Adependent transcriptional regulation by reducing genome-wide occupancy of ARID1A, thus phenocopying Arid1a deficiency in driving acinar to ductal metaplasia (ADM).

Mechanistically, we identified the Nuclear Factor of Activated T cells cytoplasmic 1 (NFATc1) transcription factor as the integrating signaling cue that elicits ARID1A genomic dissociation from chromatin in response to EGFR signaling activation, thus driving ADM. In conclusion, we demonstrate a novel EGFR-NFATC1-ARID1A regulatory axis in promoting pancreatic acinar reprogramming and provide mechanistic insights into the 
upstream epigenetic regulation of ARID1A activity. 


\section{Introduction}

\subsection{Precursor lesions of pancreatic ductal adenocarcinoma}

Pancreatic ductal adenocarcinoma (PDAC) accounts for over 95\% of the pancreatic cancer cases and is one of the most fatal diseases by having a lethality rate close to its incidence. Despite tremendous scientific efforts taken in the past 4 decades the 5-year survival has marginally increased to $8 \%-10 \%$ (Mizrahi et al. 2020). The poor survival of this malignancy, in part, is due to the failure of early stage detection. Patients mostly remain asymptomatic until having abdominal pain and jaundice, when tumors are already advanced or metastasized, thus being inoperable (Gillen et al. 2010; Jemal et al. 2005). However, even the patients who are eligible for initial resection inevitably suffer from recurrence and their 5-year-survival rate is only pro-longed to 25\% (Yeo et al. 2002; Siegel et al. 2014). These clinical characteristics raise the importance of not only identifying PDAC at earlier stage, but also dissecting the pathological events during initiation phase.

PDAC is believed to arise from noninvasive mucinous lesions (Mohammed et al. 2014). The three types of best characterized precursor lesions of this malignancy are pancreatic intraepithelial neoplasia (PanIN), intraductal papillary mucinous neoplasms (IPMN) as well as mucinous cystic neoplasm (MCN). PanIN is the most common precursor as being detected in more than $80 \%$ of the invasive PDAC (Feldmann et al. 2007). PanIN histologically progresses from the banal PanIN1 over low-grade papillary lesion with intermediate dysplasia (PanIN2), to high-grade PanIN3 showing carcinoma in situ, marked by the presence of mitotic figures and luminal necrosis (Figure 1) (Hruban et al. 2001).

The histological progression of PanIN is accompanied by a series of genetic abnormalities, almost all of which are concurrently found in the adjacent invasive pancreatic carcinoma, just with higher frequency (Maitra et al. 2005). Mutations of oncogenic KRAS is considered as the earliest molecular event for PanIN formation and is observed in approximately $90 \%$ of human pancreatic cancer cases, with G12D as its most prevalent type (Hruban et al. 2001). The KRAS proto-oncogene encodes a small ( 21KDa) GTPase which cycles between a GTP-bound active state and a GDP-bound inactive state to transmit signals to mitogen-activated protein kinase (MAPK) and PI3K/AKT pathways to regulate cell proliferation (Lohr et al. 2005). The activating mutation of KRAS impairs the intrinsic GTPase function and leads to an accumulative 
active state which persistently stimulates the downstream pathways that drive proliferation, anti-apoptosis, evasion of immune-response and hallmarks of cancer (Pylayeva-Gupta et al. 2011). However, oncogenic KRAS alone is not sufficient to propel carcinogenesis beyond initiation, the second event such as mutations in tumor suppressor genes CDKN2A/INK4A, TP53 or DPC4/SMAD4 or inflammation are required for the establishment of invasive PDAC (Klimstra et al. 1994; Cooper et al. 2013; Delpu et al. 2011).

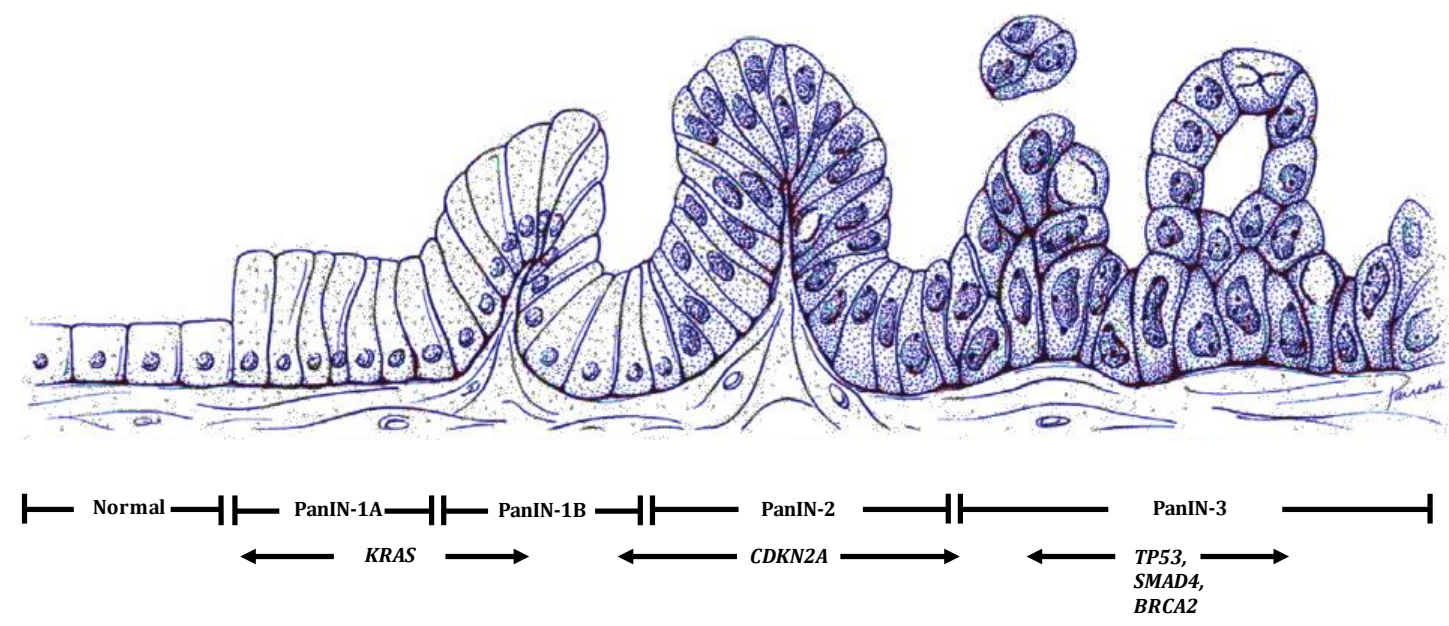

Figure1. The progression model of pancreatic epithelial neoplasia (PanIN). The progression process from histologically normal duct to low grade PanIN1 and PanIN2, finally to high grade PanIN3 (carcinoma in situ) is triggered by accumulation of specific genetic alterations (Hruban et al. 2001).

\subsection{Pancreatic acinar cell reprogramming}

Previously, only the multipotent stem cells were believed to competently differentiate into other cell types under certain stimuli/conditions. The triggering cues can be cellular events such as the activation of key transcription factors or epigenetic factors that regulate lineage trajectory or affect the expression profile of the cell (Puri et al. 2015). However, more recent experimental evidence suggests that the fate of terminally differentiated cells is more flexible as assumed previously (Graf 2011; Cherry and Daley, 2012). Specifically in the pancreas, plasticity endows the differentiated cells with the capacity to convert into other pancreatic cell types in response to injury or stress (Puri et al. 2015).

The pancreas is composed of two distinct compartments that confer the organ both 
exocrine and endocrine function (Slack 1995). The exocrine compartment is the majority of the pancreas ( $85 \%$ by mass) and its functions derive from acinar cells, which synthesize and secrete digestive enzymes (proteases, lipases, nucleases and amylase) and from duct cells, which form highly-branched epithelium to transport the digestive enzymes and bicarbonate to the intestine. The endocrine pancreas secretes hormones into the blood stream (Slack 1995; Edlund 2002; Grapin-Botton 2005). Among all the adult cell types of the pancreas, acinar cells have the highest plasticity (Stanger and Hebrok 2013). Studies using murine models showed that upon internal or external stimuli acinar cells can lose their morphological features and dedifferentiated or transdifferentiated, respectively, into an embryonic progenitor or duct-like phenotype that both express ductal markers. This process is termed acinar-to-ductal metaplasia (ADM) (Rooman and Real 2012; Stanger and Hebrok 2013). In mice, ADM can be induced by oncogenic KRAS activity and/or inflammatory signaling cues (Liou et al. 2013; Logsdon et al. 2009). Constitutive active KRAS leads to abnormalities in the transcriptional regulatory networks of cell identity (Wilson et al. 2003). Ptf1a, Mist1 and Nr5a2 genes which establish and stabilize acinar cell identity are silenced and ductal genes encoding cytokeratin 19 (Krt19), mucin 1 (Muc1) and Sry-related high-mobility group box 9 (Sox9) are upregulated (Arda et al. 2013). ADMs with constantly activated KRAS are not reversible and will continue to progress into PanINs or other dysplastic lesions and further eventually cause PDAC development (Kopp et al. 2012; Aichler et al. 2012).

\subsection{EGFR signaling}

\subsubsection{EGFR signaling cascade}

The epidermal growth factor receptor (EGFR/HER1/erbB1) belongs to a family of receptor tyrosine kinases (RTKs) which are transmembrane glycoproteins that consist of an extracellular ligand-binding domain, a short hydrophobic transmembrane domain and an intracellular receptor tyrosine kinase (RTK) domain (Schlessinger and Lemmon 2006; Hynes and Lane 2005). The known cognate ligands of EGFR include, but are not limited to EGF, transforming growth factor $\alpha(T G F-\alpha)$, amphiregulin (AR), betacellulin (BTC) and epiregulin (EPR) (Yarden and Sliwkowski 2001; Citri and Yarden 2006). Followed by the ligand-induced receptor dimerization and auto-phosphorylation, the tyrosine residues present in the kinase domain successively phosphorylate the other receptor proteins that anchored on the EGFR and subsequently initiate several downstream signaling cascades (Figure 2) (Siwak et al. 2010). The activation of downstream mitogenic events, 
such as the mitogen-activated protein kinase (MAPK), phosphatidylinositol 3-kinase (PI3K)/Akt and signal transducers and activators of transcription (STAT) signaling pathways elicit e.g. endothelial cell proliferation, growth and metastasis (Klein et al. 2004; Dawson et al. 2005).

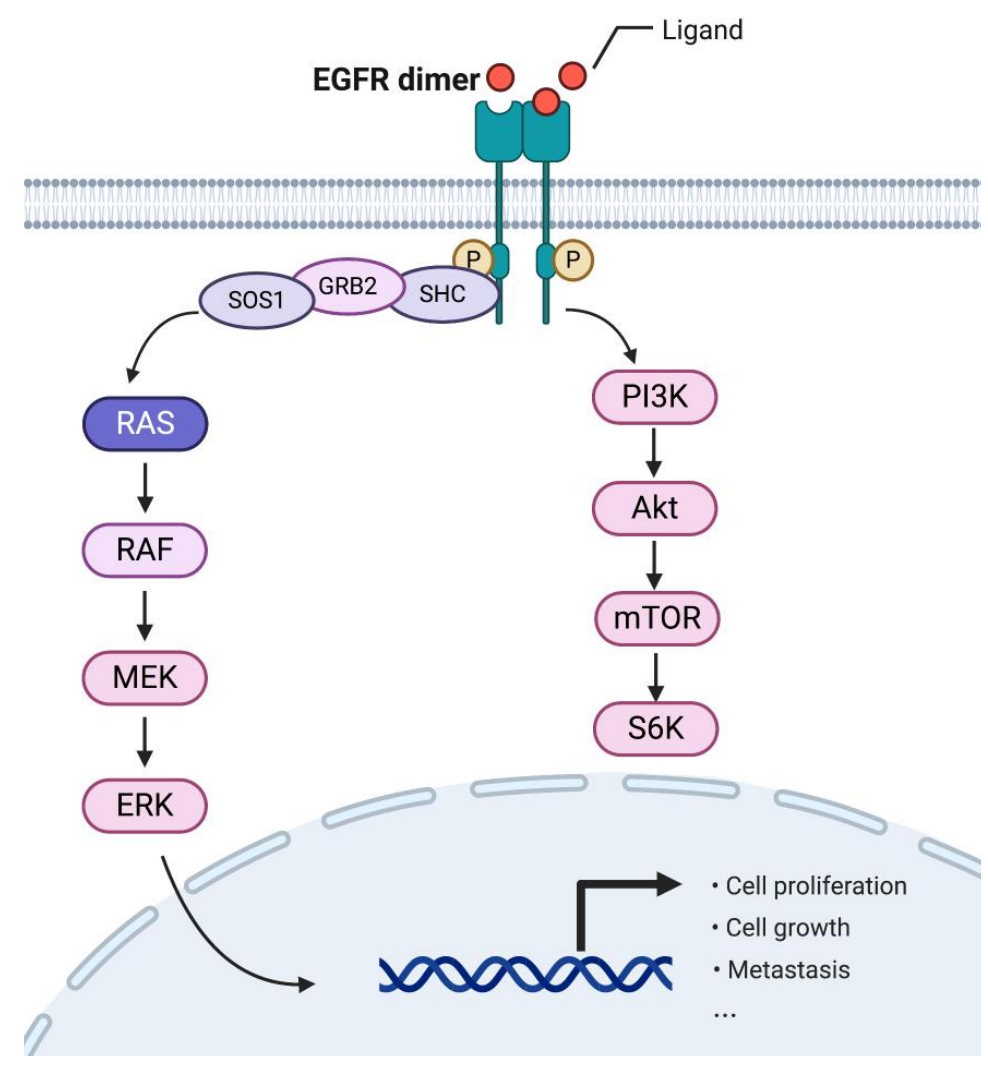

Figure 2. Conventional diagram of epidermal growth factor receptor (EGFR) signaling pathway. EGF receptor becomes activated in response to ligand stimulation and subsequently activates downstream signaling cascades such as the RAS/MAPK and PI3K/Akt pathways to promote cell proliferation, growth and metastasis.

\subsubsection{The role of EGFR in PDAC initiation}

The acquisition of mutated KRAS is considered as the primary initiator of PDAC, however, the transgenic mice harboring ubiquitous Kras mutation in pancreatic parenchymal remain largely indolent, suggesting that the activity of mutant Kras needs to be further potentiated to trigger the progression from ADM to PanIN and pancreatic cancer ( $\mathrm{Ji}$ et al. 2009). Increasing evidence showed that the signaling cascade which activates wild-type Kras is additionally required to accumulate KRAS activity to certain pathological 
threshold for PDAC progression (Ji et al. 2009; Liou et al. 2016). As the major upstream signaling activator of KRAS, EGFR signaling not only enhances oncogenic KRAS activity but also directs wild-type KRAS to the plasma membrane via GRB2/SOS where it is activated (Ardito et al. 2012; Liou et al. 2015). The Egfr depletion in Kras ${ }^{G 12 D}$ mice resulted in more than $50 \%$ reduction of active KRAS, suggesting the significance of EGFR signaling in boosting KRAS expression. EGFR signaling is frequently hyperactivated in epithelial tumors by EGFR overexpression or constitutive activation (Hemming et al. 1992). This also accounts true for pancreatic cancer, where the expression of EGFR has been correlated with in vivo aggressiveness and in vitro ADM formation (Yamanaka et al. 1993; Tobita et al. 2003; Means et al. 2005; Ardito et al. 2012; Navas et al. 2012). The leading cause of the hyper-activated EGFR signaling is the $K R A S^{G 12 D}$ activity. KRAS $S^{G 12 D}$ leads to altered mitochondrial metabolism in acinar cells and facilitates the generation of mitochondrial reactive oxygen species (mROS), which in turn activates NF-KB transcription factor. The activated NF-KB leads to activation of EGFR signaling pathway by upregulating EGFR, its ligands and Sheddase ADAM17 (Liou et al. 2016).

Oncogenic Kras activity is not sufficient to compensate EGFR activity (Ardito et al. 2012). Studies have shown that transgenic mice with TGFa overexpression-induced EGFR hyper-activity accelerate the process to metastatic PDAC (Siveke et al. 2007). EGFR activity is required for ADM formation in vitro and in vivo, since genetic ablation of Egfr or pharmacological inhibition of EGFR both strikingly eliminated $K \mathrm{ras}^{\mathrm{G12D}}$-initiated pancreatic tumorigenesis. Emerging studies have revealed the underlying mechanism of this indispensability as the non-redundant functions of EGFR and oncogenic Kras. Activated EGFR signaling also activates wild-type Kras. Together with the oncogenic Kras, wild-type Kras relays signals through downstream signaling hubs serine/threonine protein kinase D1 (PRKD1) and PI3K. PRKD1 initiates NF-KB and Notch signaling and upregulate matrix metalloproteinases (MMPs), which may contribute to PDAC initiation and tumor growth (Slapak et al. 2020; Storz 2017). Besides, EGFR signaling is found to function through alternative signaling cascade independent of KRAS. During PDAC initiation, EGFR phosphorylates MEK/ERK to promote ADM formation, bypassing G protein and RAS signaling (Lee et al. 2016). ERK phosphorylation is not affected by Kras knockdown, but rather shows strong dependency on EGFR, as shown in vivo and in vitro, and is verified as required for EGFR-mediated PDAC initiation (Lee et al. 2016; Ardito et al. 2012) 
Due to its prevalent activation observed in substantial PDAC patients, EGFR has been assumed to be a promising target in anti-cancer therapy. However, targeting EGFR has failed to show significant progress in benefiting patients with metastatic pancreatic cancer with complex reasons (Moore et al. 2007; Collison et al. 2011). The particular cues in different molecular subtypes may have impact on the yield of therapy targeting EGFR, which we will not excessively elaborate here.

\subsection{Chromatin remodeling}

The eukaryotic DNA is highly compacted into chromatin by enwinding on a nucleosome octamer of core histones H2A, H2B, H3 and H4 (Kornberg 1974; Germond et al. 1975; Luger et al. 1997). For the purpose of precisely regulating the expression network of numerous genes in a spatially and temporally restrictive manner, chromatin is partitioned into transcriptionally active and repressive regions (Urnov and Wolffe 2001). The specialization of chromatin regions and the establishment of DNA-accessibility in packaged regions require rapid rearrangement of chromatin structure. This is addressed/enabled by the participation of histone modifying enzymes and ATPdependent chromatin remodeling complexes as well as adaptor protein that are equipped with domains recognizing histone modifications (Wang et al. 2004; Saha et al. 2006; Clapier and Cairns 2009).

Common modifications of histones include the lysine acetylation, methylation, ubiquitylation and SUMOylation, the arginine methylation, and the phosphorylation of serine, threonine or tyrosine (Zentner and Henikoff 2013). Site-specific histone modifications can direct genomic-binding of chromatin remodeling complexes via interacting with the chromatin reader motifs on those complexes (Swygert and Peterson 2014). For instance, bromodomain recognizes and binds acetylated lysines, which have been described as transcription active hallmark, while chromodomain and PHD finger domain bind methylated lysines (Haynes et al. 1992; Jeanmougin et al. 1997; Patel and Wang 2013).

Conversely, the modification state of the histones is also under influence, or sometimes direct modulation of ATP-dependent chromatin remodeling enzymes, which are polymorphic complexes that remodel chromatin structure by mobilizing nucleosomes by either sliding or ejecting histones with the energy of ATP hydrolysis (Figure 3) (Cosma 
2002; Levine and Tjian 2003). Specifically, the mechanism includes the binding of the complex on nucleosome DNA, disruption of the DNA-histone contacts, induction and propagation of the DNA loop followed by recruitment of the transcriptional apparatus to the accessible sites (Saha et al. 2006). Based on the subunit composition, these complexes can be further classified into families include SWI/SNF, ISWI, NURD/Mi2/CHD and INO80, SWIR.

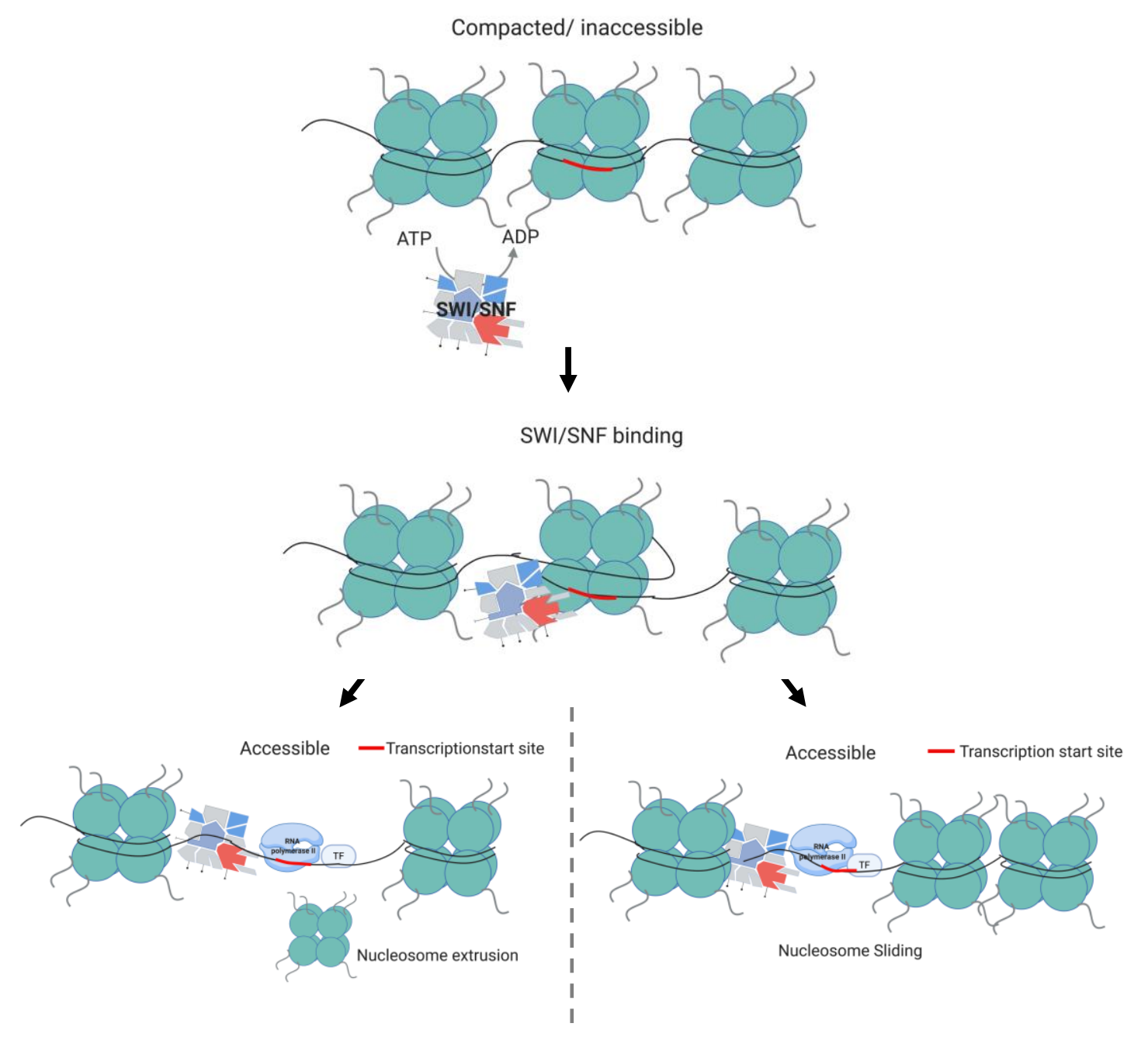

Figure 3. ATP-dependent chromatin remodeling. ATP-dependent chromatin remodeling complexes utilize the energy from ATP hydrolysis to disrupt the DNA-histone contact and remodel the compacted chromatin into a more accessible state for transcription apparatus to bind. 


\subsubsection{SWI/SNF complex}

\subsubsection{Composition and function of SWI/SNF}

Mammalian SWI/SNF (SWItch/Sucrose Non-Fermentable) complex, grouped into two subfamilies as BAF (BRM- or BRG1-associated factor) and PBAF (polybromo BRG1associated factor), are critically involved in key cellular processes including development, tissue differentiation, cell proliferation and DNA repair (Ivana et al. 2006; Romero et al. 2014; Wu 2012). Being the essential catalytic subunit of the complex, BRG1 and BRM share $75 \%$ similarity in amino-acid sequences (Roberts et al. 2004). BAF contains one of the two mutually exclusive ATPase subunits BRG1 and BRM while PBAF contains only BRG1. Apart from the ATPase subunit, other invariant core subunits including BAF47(SMARCB1/SNF5), BAF155 (SMARCC1) and BAF170 (SMARCC2) assemble into the prototypic BAF and are crucial for remodeling activity (Phelan et al. 1999). Variant accessory subunits such as BAF57 (SMARCE1), BAF60 A/B/C (SMARCD1/2/3), $\beta$-actin (ACTB) and BAF53A/B (ACTL6A/B) are responsible for the assembly, regulation and genomic targeting of the complex (Figure 4) (Wang et al. 1996). The diversity of the complex assembly is derived from having alternative family members, for examples, BAF has BAF250A/B (ARID1A/B), BAF45B/C/D (DPF1/3/2) and BRD9, while PBAF has BAF200 (ARID2), BAF45A (PHF10) BAF180 (PBRM1) and BRD7 (Mohrmann et al. 2005). Besides, the composition of the complex varies in different tissue types or development stages, confers BAF complex the functional specificity (Lickert et al. 2004; Wang et al. 2004; Wu et al. 2017). For instance, BRG1-related BAF plays an important role in smooth muscle formation while ARID2-containing PBAF is a key regulator for coronary morphogenesis (Hota and Bruneau 2016).

Since firstly discovered in Saccharomyces cerevisiae, SWI/SNF activity has been associated predominantly with activation of transcription (Utley et al. 1997; Burns and Peterson 1997; Fryer and Archer 1998). Nevertheless, mammalian SWI/SNF have also been evidenced to directly regulate transcriptional repression (Breeden and Nasmyth 1987; Martens et al. 2005). During T lymphocyte development, for instance, BAF57 and BRG1 can both silence CD4 and activate CD8 (Chi et al. 2002). In addition, BAF complexes have been reported to physically interact with members of the histone deacetylase (HDAC) family of transcriptional repressors. For instance, BRG1 interacts with HDAC to silence cyclin A (CCNA2) and elicit persistent cell cycle arrest (Zhang et al. 2000) while ARID1A-HDAC1 interaction is accountable for the acetylation status shift 
upon ARID1A depletion (Nagarajan et al. 2020). Furthermore, loss of SNF5 leads to transcriptional activation in the murine embryonic fibroblast that more genes are upregulated then being repressed (Isakoff et al. 2005). Hence, the impact of SWI/SNF activity on transcriptional regulation is much broader than admitted initially.

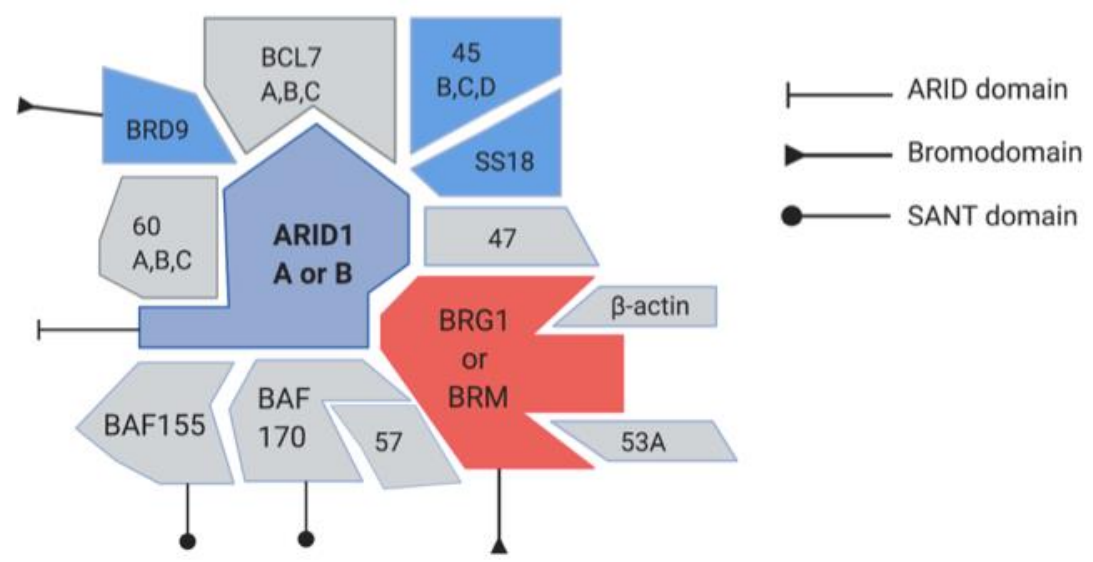

Figure 4. Mammalian SWI/SNF are polymorphic complexes. Mammalian SWI/SNF complexes are comprised of one of two catalytic ATPase subunits (either BRG1 or BRM), a set of highly conserved core subunits (BAF155, BAF170, BAF47) and variant subunits which contribute to the targeting and recruitment of the complex. The chromatin reader motifs on the complex can recognize different histone modifications.

\subsubsection{SWI/SNF and cancer}

Since the last decade, numerous exome sequencing studies have revealed the high frequency of mutations of components that constitute ATP-dependent chromatin remodeling complexes in human cancer, among which, most of the mutations occur within SWI/SNF complex. The mutations of genes encoding the 15 subunits of SWI/SNF have been found in over $20 \%$ of cancer cases, in a broad range. For instance, SWI/SNF mutations are predominant in ovarian clear-cell carcinoma, as approximate $75 \%$ of the patients harbor SWI/SNF mutations.

The dysregulation of the SWI/SNF function due to the recurrent mutations has been associated with malignant transformation, since substantial evidence indicates that several components of the complex function as tumor suppressors. The strongest evidence of BAF component for being involved in tumor suppression comes from the biological consequences of alterations affecting the core subunit BAF47 (SMARCB1). The BAF47 encoding gene is located on chromosome $22 q 11$, a region that frequently undergoes deletion or translocation in pediatric rhabdoid tumors. SMARCB1 is 
biallelically inactivated in nearly all malignant rhabdoid tumors (RTs), which is a fatal childhood malignancy that arises in the kidney, soft tissues and central nervous system, and poorly responses to treatment (Versteege et al. 1998; Rousseau-Merck et al. 1999; Biegel et al. 2000). The tumor suppressor property of Smarcb1 is confirmed by the finding in transgenic mouse models, that $30 \%$ of mice with heterozygous Smarcb1 loss developed sarcomas which closely resemble human RTs (Roberts et al. 2000). To better simulate human cancer, where the second allele of Smarcb1 is either mutated or silenced by methylation, Roberts et al. generated a mouse model with conditional biallelic Smarcb1 depletion. Smarcb1-deficient mice develop lymphomas or rhabdoid tumors with $100 \%$ penetrance at a median onset of only 10 weeks postnatal (Roberts et al. 2002). However, in adult tumors, the loss of BAF47 is not frequently detected. Instead, the defects of the catalytic subunits BRG1 and BRM are more prevalent. BRG1 deficiency is detected in $15-50 \%$ of human primary non-small-cell lung cancer (NSCLC) samples, as well as colorectal, hepatocellular, renal and pancreatic cancer (Cristofaro et al. 2001). Both $B R G 1$ and BRM gene loci exhibit loss of heterozygosity in human cancer and the coordinate loss of BRG1 and BRM is more frequent than loss of either ATPase (Eiriksdottir et al. 1995; Tripathi et al. 2003; Reisman et al. 2003). About 20\% of the established NSCLC cell lines also show dual BRG1/BRM loss and the restoration of BRG1 or BRM leads to increased nuclear size and area of attachment, as well as growth arrest (Hill et al. 2004; Glaros et al. 2007). Microarray analysis by Reisman et al. identified a collection of important cell-adhesion proteins that are under regulation of BRG1, such as SPARC, INTEGRIN A3, MMP1 (Reisman et al 2009). Besides, BRG1 and BRM link the SWI/SNF complex to Kras-induced senescence and DNA repair, the key tumor suppression mechanism, by directly interacting with BRCA1 and pRb (Tu et al. 2013). In pancreatic cancer, integration of DNA-microarray, gene expression profiling as well as exon- and transcriptome-sequencing of the cancer genome exhibited notable mutations of the three putative DNA-binding subunits (ARID1A, ARID1B, ARID2) and ATPase catalytic subunits (BRG1 and BRM) and the mutation of each subunit occur at modest frequency (2\%-10\%) (Kadoch et al. 2013; Shain et al 2011). The role of the mutation of each subunit has been shown to vary along with distinct stages of cancer, as BRG1 attenuates tumor initiation but promotes tumor progression once tumor is established, by activating a mesenchymal-like transcription landscape (Shain et al. 2011; Roy et al. 2015). 


\subsubsection{AT-rich interactive domain-containing protein $1 \mathrm{~A}$}

ARID1A (also known as BAF250a or p270) belongs to a family of proteins with a highlyconserved DNA-binding domain called ARID (AT-rich interaction domain). Although several members of this family preferentially bind AT-rich DNA sequences, genome-wide occupancy of ARID1A shows no sequence specificity in vitro (Dallas et al. 2000). ARID1A acts as nuclear protein and its expression varies during cell cycle- it accumulates in G0 followed by downregulation throughout the cell cycle (Flores-Alcantar et al. 2011).

It is believed that ARID1A contributes to BAF targeting with the aid of its functional domain. The $\mathrm{C}$ terminus of the ARID1A protein contains an LXXLL motif, which has been shown as important in facilitating interaction with nuclear hormone receptors (Heery et al. 1997). ARID1A presumably functions in recruiting BAF to the target DNA-regulatory elements by interacting with other transcription factors or co-factors through its Cterminal domain (Nie et al. 2000). Besides, although the precise function of sequenceunspecific ARID domain remains largely unknown, evidence exists that ARID1A-DNA interaction mediated by ARID domain is essential for the intact SWI/SNF function. Chandler et al. mutated the DNA-binding ARID domain in ARID1A and found an impaired nucleosome substrate binding by SWI/SNF, as well as defective cardiovascular development (Chandler et al. 2013). Since mutations in ARID domain are also found in human PDAC cases, it suggests that ARID1A-DNA contact may be required for the tumor-suppressor function of ARID1A (Wu et al. 2014).

ARID1A is the most frequently mutated SWI/SNF subunit with mutations primarily observed in solid tumors. Particularly high frequency of ARID1A mutations are detected in endometriosis-related ovarian clear cell carcinoma (OCCC) (46-57\%) and endometrioid cancer (30\%) (Jones et al. 2010; Wiegand et al. 2010). Further comprehensive sequencing studies revealed frequent somatic mutations of ARID1A in other cancer types including uterine endometrioid carcinoma (39-44\%), gastric cancer (8-29\%), bladder cancer (18.6\%), hepatocellular cancer $(13.7 \%)$, colorectal cancer (9.4\%) and pancreatic cancer (3-5\%) (Wang et al. 2011; Liang et al. 2012; Kadoch et al. 2013; Bailey et al. 2016).

The ARID1A gene is located on chromosome 1p36.11, a region that is frequently deleted in cancer (Bagchi and Alea 2008). The vast majority (>97\%) of ARID1A cancerassociated mutations are inactivating, with nonsense- or deletion mutations evenly 
distributed across the gene body, rather than enriching at hotspots (Jones et al. 2010; Wiegand et al. 2010). Besides, the mutations affecting both ARID1A alleles are less frequently detected than heterozygous mutations.

ARID1A has been characterized as a candidate tumor suppressor by the findings demonstrated in the following cellular processes, as loss of function of ARID1A contributes to the initiation or maintenance of the tumor phenotype. 1) Cellular proliferation. ARID1A significantly inhibits the growth of breast cancer cells in soft agar and ARID1A depletion accelerates proliferation (Mamo et al. 2012). In ovarian cancer research, ARID1A silencing endues non-transformed epithelial cells with high proliferation and tumorigenicity, whereas re-expression of wild-type ARID1A prominently represses cell proliferation and tumor growth in mice (Guan et al. 2011). In human pancreatic cancer epithelial cell lines, ARID1A-knockdown antagonized KRAS-induced cellular senescence and accelerates cell cycle progression (Li et al. 2017). 2) Epithelialto-mesenchymal transition (EMT). ARID1A loss leads to EMT as shown by an increase of mesenchymal genes and a reduction of epithelial genes, in renal cells as well as endometrium epithelial cells (Somsuan et al. 2019; Wilson et al. 2019). Similar finding was made in pancreatic cancer, where Arid1a deficiency leads to a more mesenchymal, migratory phenotype to $K r a s^{G 12 D} ; p 53^{K O}$ mouse models, with an enrichment of genes for EMT as revealed by gene set analysis (Wang W. et al. 2018). 3) DNA repair. SWI/SNFmediated chromatin remodeling is an integral component of the DNA damage repair (DDR machinery, which protects cells from DNA-damage-induced genomic instability and oncogenesis) (Ribeiro-Silva et al. 2019). The core catalytic subunits BRM and BRG1 have been implicated in facilitating efficient DNA double-strand break (DSB) repair by $\mathrm{H} 2 \mathrm{AX}$ phosphorylation induction ( $\mathrm{Y}-\mathrm{H} 2 \mathrm{AX})$. The binding of topoisomerase Ila (TOP2A), the topoisomerase essential for separating the entangle strands during DNA replication, requires physical interaction with ARID1A and the ATPase activity of SWI/SNF complex (Lou et al. 2005). SWI/SNF is also required in nucleotide excision repair (NER) to eliminate the helix-distorting DNA lesions induced by UV, ROS or environmental chemicals. ARID1A also participates in DNA repair. By promoting non-homologous end joining (NHEJ) at DSBs, ARID1A confers resistance to radiation and chemotherapy on lung and bone osteosarcoma cells (Watanabe et al. 2014). In endometrial carcinoma, ARID1A loss leads to mismatch-repair deficiency (Allo et al. 2014). Besides, Williamson et al. reported that $A R I D 1 A$ deficiency results in defects in topoisomerase $2 \mathrm{~A}$ and sensitizes breast cancer cells to inhibitors of ATR, the DNA damage checkpoint kinase, 
both in vivo and in vitro (Williamson et al. 2016).

In recent years, there are increasing studies that demonstrate the impact of $A R I D 1 A$ deficiency on pancreatic pathogenesis. By taking advantage of genetically engineered mouse models (GEMM) as well as the high-throughput sequencing technics, those literatures uncovered that Arid1a deficiency leads to disrupted pancreatic acinar cell homeostasis and aberrant duct morphology, resulting in acinar-to-ductal conversion and dilated ducts. Besides, Arid1a loss cooperates with oncogenic Kras in driving multilocular fluid-filled cystic lesions, which resemble human intraductal papillary mucinous neoplasm (IPMN) (Wang S.C et al. 2019; Livshits et al. 2018; Wang W et al. 2018; Kimura et al. 2018). Mechanistically, Arid1a loss-mediated acinar transdifferentiation has been characterized with reduced chromatin accessibility for acinar genes and activated transcriptional landscape that favoring ductal genes expression.

Like the ATPases of SWI/SNF complex, ARID-containing subunits also appear as two mutually exclusive proteins. The homolog of ARID1A is a protein termed ARID1B, whose mutation is less detected in cancer compared to ARID1A (Caumanns et al. 2018). Although the two BAF250 isoforms share nearly $80 \%$ amino acid homology in the ARID domains and $59 \%$ sequence identity in the entire protein, their functions are not exactly identical. The existence of SWI/SNF complexes with opposing roles via incorporation of distinct subunits were firstly discovered in 2007, when Nagl et al. showed that ARID1Acontaining BAF repress cell-cycle while ARID1B confers BAF pro-proliferative activity via associating with MYC and Cyclin E (Nagl et al. 2007). Helming et al. found that ARID1A and ARID1B co-mutations frequently occur in cancer, but at least one ARID1B allele is retained in ARID1A mutated cases. OCCC cells show vulnerabilities to ARID1B loss in the context of ARID1A mutations that loss of ARID1B destabilizes the SWI/SNF complex and impairs proliferation (Helming et al. 2014). The synthetic lethality between these two proteins is also seen in chromatin landscape regulation. ARID1A is dominant in regulating chromatin accessibility in colorectal cells and removal of ARID1A leads to substantial decrease of complex occupancy at enhancer regions. In contrast, the effect of ARID1B is only evident in the absence of ARID1A (Mathur et al. 2017; Kelso et al. 2017).

\subsection{Aims of the study}

Recent genome-wide sequencing efforts remarkably highlighted the role of chromatin 
regulation in pancreatic cancer development. Particularly, mutations in genes encoding subunits of SWI/SNF are repeatedly discovered in genomic analysis (Bailey et al. 2016; Centore et al. 2020). Among these, the ARID1A subunit of SWI/SNF is recurrently mutated in a broad range of human malignancies, including pancreatic cancer.

While the functional implications of Arid1a deficiency in Kras-driven pancreatic carcinogenesis have been studied intensively, findings characterizing the consequences of sole Arid1a deficiency in the absence of oncogenic Kras are conflicting (Wang S.C et al. 2019; Livshits et al. 2018; Ferri-Borgogno et al. 2019). We propose, that Arid1a deficiency critically impacts on acinar reprogramming and hypothesize that the chromatin remodeling protein's function and activity is critically influenced by the activity of signaling pathways which also play a pivotal role in acinar cell plasticity and pancreatic carcinogenesis. Given its aforementioned implications in acinar to ductal metaplasia and PDAC development, we concentrated our analyses on the crosstalk of ARID1Adependent chromatin regulation and EGFR signaling in the pancreas.

Taking advantage of transgenic mouse models of pancreatic Arid1a deficiency and by performing in depth transcriptional and chromatin-associated analyses, we aimed at:

1) To dissect the functional implications of Arid1a deficiency in acinar reprogramming/acinar to ductal transdifferentiation.

2) To elucidate the mechanistic consequences of Arid1a deficiency on transcriptome and chromatin landscape.

3) To investigate the interplay of EGFR signaling and Arid1a deficiency in acinar to ductal metaplasia.

4) To characterize the consequences of EGFR signaling on ARID1A-controlled gene transcription. 


\section{Material and Methods}

\subsection{Material}

\subsubsection{Lab equipment}

Table 1 Lab equipment.

\begin{tabular}{|c|c|}
\hline Equipment & Company \\
\hline Arium $®$ pro ultrapure water system & Sartorius, Göttingen, Germany \\
\hline Aspirator with trap flask (Grant bio FTA-1) & Grant Instruments, Cambridge, UK \\
\hline Benchtop Orbital Shaker (MaxQTM 4450) & $\begin{array}{l}\text { Thermo Fisher Scientific, Waltham, } \\
\text { MA, USA }\end{array}$ \\
\hline Bioanalyser 2100 & $\begin{array}{l}\text { Agilent Technologies, Santa Clara, } \\
\text { California, USA }\end{array}$ \\
\hline $\begin{array}{l}\text { Biological safety cabinet, class II (Thermo } \\
\text { Scientific }^{\text {TM }} \text { Safe 2020) }\end{array}$ & $\begin{array}{l}\text { Thermo Electron LED GmbH, } \\
\text { Langenselbold, Germany }\end{array}$ \\
\hline Bioruptor $^{\circledR}$ Pico sonication device & Diagenode, Liege, Belgium \\
\hline Centrifuge - Perfectspin 24R (refrigerated) & $\begin{array}{l}\text { PEQLAB Biotechnologie } \mathrm{GmbH} \text {, } \\
\text { Erlangen, Germany }\end{array}$ \\
\hline $\begin{array}{l}\text { Centrifuge - Heraeus }{ }^{T M} \text { Megafuge } 16 / \text { Multifuge } \\
\text { X1R }\end{array}$ & $\begin{array}{l}\text { Thermo Fisher Scientific, Waltham, } \\
\text { MA, USA }\end{array}$ \\
\hline $\mathrm{CO}_{2}$ incubator (HERAcell $\left.{ }^{\circledR} 240 \mathrm{i}\right)$ & $\begin{array}{l}\text { Thermo Fisher Scientific, Waltham, } \\
\text { MA, USA }\end{array}$ \\
\hline Cold plate - Histocore Arcadia c & Leica Biosystem, Wetzlar, Germany \\
\hline Fluid Aspiration System - BVC Control & $\begin{array}{l}\text { Vacuubrand GmbH + Co. KG, } \\
\text { Wertheim, Germany }\end{array}$ \\
\hline Fluorescence microscope - DMi8 automated & $\begin{array}{l}\text { Leica Microsystems CMS GmbH, } \\
\text { Wetzlar, Germany }\end{array}$ \\
\hline Freezer $-20^{\circ} \mathrm{C}$ & Liebherr, Bulle, Switzerland \\
\hline Freezer $-80^{\circ} \mathrm{C}$ Ultra low temperature & Sanyo electric Co. Ltd Japan \\
\hline $\begin{array}{l}\text { Heated Paraffin Embedding Module - EG1150 H } \\
\text { with cold plate }\end{array}$ & $\begin{array}{l}\text { Leica Biosystems Nussloch } \mathrm{GmbH} \text {, } \\
\text { Nussloch, Germany }\end{array}$ \\
\hline Horizontal gel electrophoresis system & $\begin{array}{l}\text { PEQLAB Biotechnologie } \mathrm{GmbH}, \\
\text { Erlangen, Germany }\end{array}$ \\
\hline Ice flaker - AF80 & Scotsman, Edinburgh, UK \\
\hline INTAS UV system & $\begin{array}{l}\text { Intas Science Imaging Instruments } \\
\mathrm{GmbH} \text {, Göttingen, Germany }\end{array}$ \\
\hline Lab balance - PCB 2000 - 1 & $\begin{array}{l}\text { Kern \& Sohn GmbH, Balingen, } \\
\text { Germany }\end{array}$ \\
\hline Lab balance - PT210 / universal PRO11 & Sartorius AG, Göttingen, Germany \\
\hline
\end{tabular}




\begin{tabular}{|c|c|}
\hline $\begin{array}{l}\text { Liquid nitrogen cell storage canister - Biocane }{ }^{\mathrm{TM}} \\
47\end{array}$ & $\begin{array}{l}\text { Thermo Fisher Scientific, Waltham, } \\
\text { MA, USA }\end{array}$ \\
\hline Magnetic stirrer - RH basic & $\begin{array}{l}\text { IKA®-Werke GmbH \& Co. KG, Staufen, } \\
\text { Germany }\end{array}$ \\
\hline Microplate reader PHOmo / LUmo & $\begin{array}{l}\text { Autobio Labtec Instruments Co. Ltd., } \\
\text { Zhengzhou, China }\end{array}$ \\
\hline Microscope - Axiovert 25 & Carl Zeiss AG, Oberkochen, Germany \\
\hline Microscope - BX43F / CKX53SF & Olympus Corporation, Tokyo, Japan \\
\hline Microwave - NN-E209W & $\begin{array}{l}\text { Panasonic Marketing Europe GmbH, } \\
\text { Hamburg, Germany }\end{array}$ \\
\hline Mini-PROTEIN ${ }^{\circledR}$ Tetra Electrophoresis Cell & Bio Rad Laboratories, Hercules, USA \\
\hline Multipette $®$ Plus & Eppendorf, Hamburg, Germany \\
\hline Nano Photometer - P330 & $\begin{array}{l}\text { Intas Science Imaging Instruments } \\
\text { GmbH, Göttingen, Germany }\end{array}$ \\
\hline Neubauer hemocytometer & Assistant, Sondheim/Rhön, Germany \\
\hline pH meter - FiveEasy ${ }^{\top M}$ Plus FEP20 & $\begin{array}{l}\text { Mettler-Toledo AG, Schwerzenbach, } \\
\text { Switzerland }\end{array}$ \\
\hline Plate spinner - PerfectSpin P & $\begin{array}{l}\text { PEQLAB Biotechnologie } \mathrm{GmbH}, \\
\text { Erlangen, Germany }\end{array}$ \\
\hline PowerPac ${ }^{\top M}$ HC High-Current Power Supply & $\begin{array}{l}\text { Bio-Rad Laboratories GmbH, Munich, } \\
\text { Germany }\end{array}$ \\
\hline Qubit Fluorometer - Q32854 & Invitrogen $\mathrm{GmbH}$, Karlsruhe, Germany \\
\hline Refrigerator $4^{\circ} \mathrm{C}$ & Liebherr, Bulle, Switzerland \\
\hline Rotary Microtome - RM2265 & $\begin{array}{l}\text { Leica Biosystems Nussloch GmbH, } \\
\text { Nussloch, Germany }\end{array}$ \\
\hline Shandon coverplate ${ }^{\mathrm{TM}}$ with cadenza system & Thermo Scientific, Waltham, USA \\
\hline Sprout $^{\circledR}$ plus mini centrifuge & $\begin{array}{l}\text { Heathrow Scientific, Vernon Hills, IL, } \\
\text { USA }\end{array}$ \\
\hline StepOnePlus ${ }^{\mathrm{TM}}$ Real-Time PCR System & Thermo Scientific, Waltham, USA \\
\hline Techne Dri-Block DB-2a Analogue Heater & Techne, Staffordshire, UK \\
\hline Thermo cycler - T100 & $\begin{array}{l}\text { Bio-Rad Laboratories GmbH, Munich, } \\
\text { Germany }\end{array}$ \\
\hline Thermomixer Compact 5350 / 5436 Mixer & Eppendorf AG, Hamburg, Germany \\
\hline Tissue dehydration machine & Leica Biosystems, Wetzlar, Germany \\
\hline Tissue float bath & $\begin{array}{l}\text { Gesellschaft für Labortechnik GmbH, } \\
\text { Burgwedel, Germany }\end{array}$ \\
\hline Tran-Blot Turbo Transfer System & Bio Rad Laboratories, Hercules, USA \\
\hline
\end{tabular}




\begin{tabular}{|l|l|}
\hline Vortexer - Heidolph Reax 2000 & $\begin{array}{l}\text { Heidolph Instruments GmbH \& Co. KG, } \\
\text { Schwabach, Germany }\end{array}$ \\
\hline Waterbath - WNB 14 & $\begin{array}{l}\text { Memmert GmbH \& Co. KG, } \\
\text { Schwabach, Germany }\end{array}$ \\
\hline Weighing balance & Sartorius AG, Göttingen, Germany \\
\hline
\end{tabular}

\subsubsection{Consumables}

Table 2: Consumables.

\begin{tabular}{|c|c|}
\hline Consumables & Company \\
\hline $\begin{array}{l}\text { Adhesion slides - SuperFrost }{ }^{\circledR} \text { Plus } \\
\text { (for IHC) }\end{array}$ & $\begin{array}{l}\text { Gerhard Menzel B.V. \& Co.KG, } \\
\text { Braunschweig, Germany }\end{array}$ \\
\hline Cell scraper $25 \mathrm{~cm}$ & Sarstedt, Inc., Newton, NC, USA \\
\hline Centrifuge tube $15 / 50 \mathrm{ml}$ & Sarstedt, Nümbrecht, Germany \\
\hline Cell strainer $-100 \mu \mathrm{m}$ & Falcon, Durham, USA \\
\hline Cryo pure vial & Sarstedt, Nümbrecht, Germany \\
\hline $\begin{array}{l}\text { Disposable pipette, } \\
\text { sterile } 2 \text { / } 5 \text { / } 10 \text { / } 25 \text { / } 50 \mathrm{ml}\end{array}$ & $\begin{array}{l}\text { Greiner bio-one, Frickenhausen, } \\
\text { Germany }\end{array}$ \\
\hline Embedding cassettes & $\begin{array}{l}\text { Th. Geyer GmbH \& Co. KG, } \\
\text { Renningen, Germany }\end{array}$ \\
\hline Folded paper filters, $185 \mathrm{~mm}$ & $\begin{array}{l}\text { Schleicher \& Schuell BioScience } \\
\text { GmbH, Dassel, Germany }\end{array}$ \\
\hline Menzel-Glaser coverslips (24 x 32mm) & $\begin{array}{l}\text { Thermo Fisher Scientific, Darmstadt, } \\
\text { Germany }\end{array}$ \\
\hline $\begin{array}{l}\text { Micro Amp }{ }^{\circledR} \text { fast optical } 96 \text {-well reaction plate } \\
(0.1 \mathrm{ml}) \text { - Applied biosystems }{ }^{\circledR}\end{array}$ & $\begin{array}{l}\text { Life Technologies Corporation, } \\
\text { Carlsbad, CA, USA }\end{array}$ \\
\hline Microtest plate 96 well & $\begin{array}{l}\text { Sarstedt AG \& Co., Nuembrecht, } \\
\text { Germany }\end{array}$ \\
\hline Microtome blade - Feather ${ }^{\circledR}$ & pfm medical AG, Cologne, Germany \\
\hline Microtube $0.5 / 1.5 / 2.0 \mathrm{ml}$ & $\begin{array}{l}\text { Sarstedt AG \& Co., Nuembrecht, } \\
\text { Germany }\end{array}$ \\
\hline Nitrocellulose blotting membrane $0.45 \mu \mathrm{m}$ & $\begin{array}{l}\text { GE Healthcare Europe GmbH, } \\
\text { Freiburg, Germany }\end{array}$ \\
\hline Optical adhesive covers (applied biosystems $®$ ) & $\begin{array}{l}\text { Life Technologies Holdings Pte. Ltd., } \\
\text { Singapore }\end{array}$ \\
\hline Parafilm® & $\begin{array}{l}\text { Pechiney Plastic Packiging, Inc., } \\
\text { Menasha, WI, USA }\end{array}$ \\
\hline PCR tubes $200 \mu \mathrm{l}$ & $\begin{array}{l}\text { Sarstedt AG \& Co., Nuembrecht, } \\
\text { Germany }\end{array}$ \\
\hline
\end{tabular}




\begin{tabular}{|l|l|}
\hline Pipette tips (TipOne $\AA)$ 10 / $200 / 1000 \mu \mathrm{l}$ & $\begin{array}{l}\text { STARLAB International GmbH, } \\
\text { Hamburg, Germany }\end{array}$ \\
\hline Scalpel & $\begin{array}{l}\text { Feather Safety Razor Co., LTD, Osaka, } \\
\text { Japan }\end{array}$ \\
\hline Syringe $5 \mathrm{ml}, 10 \mathrm{ml}$ & $\begin{array}{l}\text { B. Braun Melsungen AG, Melsungen, } \\
\text { Germany }\end{array}$ \\
\hline Sterile syringe filter $(0.2 \mu \mathrm{m})$ & $\begin{array}{l}\text { Labsolute, Th.Geyer ingredients GmbH } \\
\text { \& Co.KG, Höxter, Germany }\end{array}$ \\
\hline TC dish 100 & $\begin{array}{l}\text { Sarstedt AG \& Co., Nuembrecht, } \\
\text { Germany }\end{array}$ \\
\hline TC flask T25, T75, T175 & $\begin{array}{l}\text { Sarstedt AG \& Co., Nuembrecht, } \\
\text { Germany }\end{array}$ \\
\hline TC multiwell plates $(24 / 12$ / 6 wells $)$ & $\begin{array}{l}\text { Greiner bio-one, Frickenhausen, } \\
\text { Germany }\end{array}$ \\
\hline
\end{tabular}

\subsubsection{Chemical and reagents}

Table 3: Chemical and reagents.

\begin{tabular}{|l|c|l|}
\hline \multicolumn{1}{|c|}{ Chemical / Reagents } & $\begin{array}{c}\text { Catalogue } \\
\text { number }\end{array}$ & \multicolumn{1}{c|}{ Company } \\
\hline 6x DNA Loading Dye & R0611 & $\begin{array}{l}\text { Thermo Fisher Scientific GmbH, } \\
\text { Dreieich, Germany }\end{array}$ \\
\hline 100x SYBR Green I & S-7563 & $\begin{array}{l}\text { Invitrogen GmbH, Karlsruhe, } \\
\text { Germany }\end{array}$ \\
\hline Acetic acid 100\% p.A. & A0820 & $\begin{array}{l}\text { AppliChem GmbH, Darmstadt, } \\
\text { Germany }\end{array}$ \\
\hline Agarose & AG02 & $\begin{array}{l}\text { Nippon Genetics Europe GmbH, } \\
\text { Düren, Germany }\end{array}$ \\
\hline Agarose A Beads, fast flow & $16-156$ & Merck Millipore, Billerica, USA \\
\hline Agarose G Beads, fast flow & $16-266$ & Merck Millipore, Billerica, USA \\
\hline Alcian blue 8GX solution & 66011 & Sigma-Aldrich, St. Louis, USA \\
\hline Ampicillin & K029.2 & $\begin{array}{l}\text { Carl Roth GmbH + Co. KG, } \\
\text { Karlsruhe, Germany }\end{array}$ \\
\hline B-Mercaptoethanol & 444203 & Merck, Darmstadt, Germany \\
\hline Bradford protein assay & 5000006 & $\begin{array}{l}\text { Bio-Rad Laboratories GmbH, } \\
\text { Neuberg, Germany }\end{array}$ \\
\hline Bovine serum Albumin & A9647 & Sigma-Aldrich, St. Louis, USA \\
\hline Chloroform p.a. CHEMSOLUTE ${ }^{\circledR}$ & 2409.2500 & $\begin{array}{l}\text { Th. Geyer GmbH \& Co. KG, } \\
\text { Renningen, Germany }\end{array}$ \\
\hline
\end{tabular}




\begin{tabular}{|c|c|c|}
\hline Citric acid monohydrate & 3958.1 & $\begin{array}{l}\text { Carl Roth GmbH + Co. KG, } \\
\text { Karlsruhe, Germany }\end{array}$ \\
\hline Cyclosporine A (CsA) & $59865-13-3$ & Sigma-Aldrich, St. Louis, USA \\
\hline Dimethyl sulfoxide (DMSO) & D8418 & Sigma-Aldrich, St. Louis, USA \\
\hline EDTA & 324503 & Merck Millipore, Billerica, USA \\
\hline EGTA & E3889 & Sigma-Aldrich, St. Louis, USA \\
\hline Ethanol absolute p.a. & 2246 & $\begin{array}{l}\text { Th. Geyer GmbH \& Co. KG, } \\
\text { Renningen, Germany }\end{array}$ \\
\hline Eosin $\mathrm{Y}$ solution aqueous & HT110232 & Sigma-Aldrich, St. Louis, USA \\
\hline Epidermal growth factor (hEGF) & E9644 & Sigma-Aldrich, St. Louis, USA \\
\hline $\begin{array}{l}\text { Ethylene Glyco bis (succinimidyl } \\
\text { succinate) (EGS) }\end{array}$ & 21565 & $\begin{array}{l}\text { Thermo Fisher Scientific GmbH, } \\
\text { Dreieich, Germany }\end{array}$ \\
\hline Formaldehyde (37\%) & 8775 & Sigma-Aldrich, St. Louis, USA \\
\hline $\begin{array}{l}\text { Formaldehyde solution 4\%, } \\
\text { buffered, } \mathrm{pH} 6.9\end{array}$ & 1.00496 .5000 & Merck KGaA, Darmstadt, Germany \\
\hline Gene 100bp DNA ladder & MWD100 & $\begin{array}{l}\text { Nippon Genetics Europe, Dueren, } \\
\text { Germany }\end{array}$ \\
\hline Gene ruler 1kb DNA ladder & SM0311 & $\begin{array}{l}\text { Thermo Fisher Scientific GmbH, } \\
\text { Dreieich, Germany }\end{array}$ \\
\hline Glycine PUFFERAN $® \geq 99 \%$ & 3908.2 & $\begin{array}{l}\text { Carl Roth GmbH Co. KG, Karlsruhe, } \\
\text { Germany }\end{array}$ \\
\hline Glycerol & 3783.1 & $\begin{array}{l}\text { Carl Roth GmbH Co. KG, Karlsruhe, } \\
\text { Germany }\end{array}$ \\
\hline Hematoxylin & 51275 & Sigma-Aldrich, St. Louis, USA \\
\hline $\begin{array}{l}\text { Hydrogen peroxide } 30 \% \\
\left(\text { ROTIPURAN }^{\circledR}\right)\end{array}$ & 8070.2 & $\begin{array}{l}\text { Carl Roth GmbH Co. KG, Karlsruhe, } \\
\text { Germany }\end{array}$ \\
\hline $\begin{array}{l}\text { iTaq Universal SYBR Green } \\
\text { Supermix }\end{array}$ & $172-5125$ & $\begin{array}{l}\text { Bio-Rad Laboratories GmbH, } \\
\text { Neuberg, Germany }\end{array}$ \\
\hline $\begin{array}{l}\text { LB Broth (Miller), Microbial } \\
\text { growth powder medium }\end{array}$ & L3522 & Sigma-Aldrich, St. Louis, USA \\
\hline Lipofectamine ${ }^{\circledR} 2000$ reagent & 11668027 & Thermo Fisher Scientific, USA \\
\hline Lipofectamine ${ }^{\circledR}$ RNAiMAX & 13778075 & Thermo Fisher Scientific, USA \\
\hline Lithium chloride & 27026 & Sigma-Aldrich, St. Louis, USA \\
\hline Midori green advanced DNA & MG04 & Nippon Genetics Europe GmbH, \\
\hline
\end{tabular}




\begin{tabular}{|c|c|c|}
\hline stain & & Dueren, Germany \\
\hline Milk powder & T145.4 & $\begin{array}{l}\text { Carl Roth GmbH + Co. KG, } \\
\text { Karlsruhe, Germany }\end{array}$ \\
\hline Nuclear fast red & $\mathrm{H}-3403$ & $\begin{array}{l}\text { Vector Laboratories, Inc., } \\
\text { Burlingame, USA }\end{array}$ \\
\hline Paraffin & $17932 \mathrm{~A}$ & $\begin{array}{l}\text { Engelbrecht GmbH, Edermünde/ } \\
\text { Besse, Germany }\end{array}$ \\
\hline PBS Dulbecco, powder & L182-50 & Biochrom GmbH, Berlin, Germany \\
\hline $\begin{array}{l}\text { Roti }^{\circledR} \text { Phenol/ chloroform/ } \\
\text { isoamylalcohol }(25: 24: 1)\end{array}$ & A156.1 & $\begin{array}{l}\text { Carl Roth GmbH + Co. KG, } \\
\text { Karlsruhe, Germany }\end{array}$ \\
\hline $\begin{array}{l}\text { Pierce TM ECL Western Blotting } \\
\text { Substrate }\end{array}$ & 32209 & $\begin{array}{l}\text { Thermo Fisher Scientific GmbH, } \\
\text { Dreieich, Germany }\end{array}$ \\
\hline $\begin{array}{l}\text { Phenylmethylsulfonyl fluoride } \\
\text { (PMSF) }\end{array}$ & $78830-56$ & Sigma-Aldrich, St. Louis, USA \\
\hline Ponceau S solution & P7071 & Sigma-Aldrich, St. Louis, USA \\
\hline $\begin{array}{l}\text { Protease inhibitor cocktail } \\
\text { cOmplete }^{\mathrm{TM}}\end{array}$ & 11836170001 & $\begin{array}{l}\text { Roche Diagnostics GmbH, } \\
\text { Mannheim, Germany }\end{array}$ \\
\hline Protein prestained ruler & 26617 & Thermo Fisher Scientific, USA \\
\hline Roti $^{\circledR}$ Mount for IHC & HP68.2 & $\begin{array}{l}\text { Carl Roth GmbH Co. KG, Karlsruhe, } \\
\text { Germany }\end{array}$ \\
\hline Roticlear ${ }^{\circledR}$ for histology & A538.2 & $\begin{array}{l}\text { Carl Roth GmbH + Co. KG, } \\
\text { Karlsruhe, Germany }\end{array}$ \\
\hline siLentFect TM Lipid reagent & $170-3362$ & $\begin{array}{l}\text { Bio-Rad Laboratories } \mathrm{GmbH} \text {, } \\
\text { Neuberg, Germany }\end{array}$ \\
\hline Sodium dodecyl sulfate (SDS) & 0183.3 & $\begin{array}{l}\text { Carl Roth GmbH + Co. KG, } \\
\text { Karlsruhe, Germany }\end{array}$ \\
\hline Sodium fluoride & 201154 & Sigma-Aldrich, St. Louis, USA \\
\hline Sodium orthovanadate & S6508 & Sigma-Aldrich, St. Louis, USA \\
\hline Sodium deoxycholate & D6750 & Sigma-Aldrich, St. Louis, USA \\
\hline Sucrose & S7903 & Sigma-Aldrich, St. Louis, USA \\
\hline $\begin{array}{l}\text { Trans-Blot Turbo } 5 x \text { transfer } \\
\text { buffer }\end{array}$ & 10026938 & $\begin{array}{l}\text { Bio-Rad Laboratories GmbH, } \\
\text { Neuberg, Germany }\end{array}$ \\
\hline TRIS PUFFERAN ${ }^{\circledR}$ & 4855.2 & $\begin{array}{l}\text { Carl Roth GmbH + Co. KG, } \\
\text { Karlsruhe, Germany }\end{array}$ \\
\hline Triton $X^{\circledR}-100$ & 3051.4 & $\begin{array}{l}\text { Carl Roth GmbH + Co. KG, } \\
\text { Karlsruhe, Germany }\end{array}$ \\
\hline TRIzol ${ }^{\circledR}$ reagent - ambion & 15596018 & Carlsbad, CA, USA \\
\hline
\end{tabular}




\begin{tabular}{|l|l|l|}
\hline Tween $^{\circledR} 20$ & $9005-64-5$ & Sigma-Aldrich, St. Louis, USA \\
\hline
\end{tabular}

Table 4: Kits.

\begin{tabular}{|l|c|l|}
\hline \multicolumn{1}{|c|}{ Kits } & $\begin{array}{c}\text { Catalogue } \\
\text { Number }\end{array}$ & \multicolumn{1}{c|}{ Company } \\
\hline Bioanalyzer DNA Kit & $5076-4626$ & $\begin{array}{l}\text { Agilent Technologies, Santa Clara, } \\
\text { California, USA }\end{array}$ \\
\hline CHIP-IT High Sensitivity ${ }^{\circledR}$ Kit & 53040 & Active Motif, La Hulpe, Belgium \\
\hline ImmPACT ${ }^{\text {TM }}$ DAB & SK-4105 & $\begin{array}{l}\text { Vector Laboratories, Inc., } \\
\text { Burlingame, USA }\end{array}$ \\
\hline iScript cDNA Synthesis Kit & 1708890 & $\begin{array}{l}\text { Bio-Rad Laboratories GmbH, } \\
\text { Neuberg, Germany }\end{array}$ \\
\hline MinElute PCR Purification Kit & 28006 & QIAGEN GmbH, Hilden, Germany \\
\hline MicroPlex Library Preparation v2 & C05010012 & Diagenode, Liege, Belgium \\
\hline Nextera ${ }^{\circledR}$ DNA Library Prep Kit & 20018704 & Illumina, San Diego, USA \\
\hline $\begin{array}{l}\text { NucleoBond Xtra Maxi kit for } \\
\text { plasmid DNA }\end{array}$ & 740424.50 & $\begin{array}{l}\text { Macherey Nagel GmbH \& Co. KG, } \\
\text { Dueren, Germany }\end{array}$ \\
\hline Truseq RNA Library Prep Kit v2 & RS-122-2001 & Illumina, San Diego, USA \\
\hline $\begin{array}{l}\text { VECTASTAIN }{ }^{\circledR} \text { Elite }{ }^{\circledR} \text { ABC HRP Kits } \\
\text { (Peroxidase, Rabbit / Mouse IgG) }\end{array}$ & PK-6101/ & $\begin{array}{l}\text { Vector Laboratories, Inc., } \\
\text { Burlingame, USA }\end{array}$ \\
\hline
\end{tabular}




\subsubsection{Antibodies and enzymes}

\subsubsection{Antibodies}

Table 5: Antibodies used for Immunohistochemistry.

\begin{tabular}{|c|c|c|c|}
\hline Antibodies & Dilution & $\begin{array}{c}\text { Catalogue } \\
\text { Number }\end{array}$ & Company \\
\hline ARID1A & $1: 750$ & 12354 & $\begin{array}{c}\text { Cell Signaling Technology, Inc., } \\
\text { Danvers, MA, USA }\end{array}$ \\
\hline AMYLASE & $1: 1000$ & sc-46657 & $\begin{array}{c}\text { Santa Cruz Biotechnology, Inc., } \\
\text { Dallas, Texas, USA }\end{array}$ \\
\hline CD45 & $1: 50$ & 550539 & BD Biosciences, San Jose, CA \\
\hline CK19 & $1: 150$ & Ab15463 & Abcam plc, Cambridge, UK \\
\hline EGFR & $1: 300$ & sc-373746 & $\begin{array}{c}\text { Santa Cruz Biotechnology, Inc., } \\
\text { Dallas, Texas, USA }\end{array}$ \\
\hline MIST1 & $1: 150$ & 14896 & $\begin{array}{c}\text { Cell Signaling Technology, Inc., } \\
\text { Danvers, MA, USA }\end{array}$ \\
\hline
\end{tabular}

Table 6: Antibodies used in Western Blot.

\begin{tabular}{|c|c|c|l|}
\hline Antibodies & Dilution & $\begin{array}{c}\text { Catalogue } \\
\text { Number }\end{array}$ & \multicolumn{1}{|c|}{ Company } \\
\hline ARID1A & $1: 1000$ & 12354 & $\begin{array}{l}\text { Cell Signaling Technology, } \\
\text { Inc., Danvers, MA, USA }\end{array}$ \\
\hline B-actin & $1: 40000$ & A3854 & Sigma-Aldrich, St. Louis, USA \\
\hline HDAC1 & $1: 800$ & $06-720$ & Merck Millipore, Billerica, USA \\
\hline NFATc1 & $1: 500$ & sc7294 & $\begin{array}{l}\text { Santa Cruz Biotechnology, } \\
\text { Inc., Dallas, Texas, USA }\end{array}$ \\
\hline Anti-rabbit (IgG) HRP & $1: 10000$ & 7074 & $\begin{array}{l}\text { Cell Signaling Technology, } \\
\text { Inc., Danvers, MA, USA }\end{array}$ \\
\hline Anti-mouse (IgG) HRP & $1: 10000$ & 7076 & \multicolumn{2}{|l}{}
\end{tabular}

Table 7: Antibodies used in Chromatin Immunoprecipitation.

\begin{tabular}{|c|c|l|}
\hline Antibodies & $\begin{array}{c}\text { Catalogue } \\
\text { Number }\end{array}$ & \multicolumn{1}{c|}{ Company } \\
\hline ARID1A & 12354 & $\begin{array}{l}\text { Cell Signaling Technology, Inc., Danvers, } \\
\text { MA, USA }\end{array}$ \\
\hline H3K27ac & GTX128944 & GeneTex, Inc, CA, USA \\
\hline NFATc1 & ab2796 & Abcam plc, Cambridge, UK \\
\hline Mouse IgG & C15400001-15 & Diagenode, Liege, Belgium \\
\hline Rabbit IgG & C15410206 & \\
\hline
\end{tabular}




\subsubsection{Enzymes}

Table 8: Enzymes used in this study.

\begin{tabular}{|c|c|l|}
\hline Enzymes & $\begin{array}{c}\text { Catalogue } \\
\text { Number }\end{array}$ & \multicolumn{1}{|c|}{ Company } \\
\hline $\begin{array}{c}\text { Micrococcal nuclease from } \\
\text { Staphylococcus aureus }\end{array}$ & $\mathrm{N} 3755$ & Sigma-Aldrich, St. Louis, USA \\
\hline Proteinase K & $\mathrm{A} 4392,0005$ & Panreac AppliChem \\
\hline $\begin{array}{c}\text { SuperScript } \\
\text { TM II Reverse } \\
\text { Transcriptase }\end{array}$ & 18064022 & $\begin{array}{l}\text { Thermo Fisher Scientific GmbH, Dreieich, } \\
\text { Germany }\end{array}$ \\
\hline RNAse A & R6513 & Sigma-Aldrich, St. Louis, USA \\
\hline
\end{tabular}

\subsubsection{Nucleic acids}

\subsubsection{SiRNA oligonucleotides}

Table 9: siRNAs used in the study

\begin{tabular}{|c|c|c|c|}
\hline siRNAs & $\begin{array}{c}\text { Catalogue } \\
\text { Number }\end{array}$ & Sequence & Company \\
\hline ON-TRGETplus & $\begin{array}{c}\text { J-017263-07- } \\
\text { ARID1A siRNA \#7 }\end{array}$ & GCAACGACAUGAUUCCUAU & $\begin{array}{c}\text { Dharmacon } \\
\text { Inc., Lafayette, } \\
\text { CO, USA }\end{array}$ \\
\hline ON-TRGETplus & $\begin{array}{c}\text { J-017263-08- } \\
\text { ARID1A siRNA \#8 }\end{array}$ & GGACCUCUAUCGCCUCUAU & \\
\hline
\end{tabular}

\subsubsection{Primers}

Table 10: qPCR primers for mRNA expression detection (All mouse species).

\begin{tabular}{|c|c|c|}
\hline Name & Forward primer sequence & Reverse primer sequence \\
\hline Amyl1 & GCAAGTGGAGGTATCGAGAAC & CTGCTACGCCAATGTCAATG \\
\hline Arid1a & ACAAGGCAGATGGAACACC & CTCTGTGAAGGCCAGGTAC \\
\hline Bhlhe40 & AGCGAAGACAGCAAGGAAACT & ACGTAAGCTCCAGAACCACTG \\
\hline Fam3c & CTATTTGCTCGATCCGCGCT & CGCCTTTGAGATCCCACACT \\
\hline Foxa3 & GTAGAGAGACCGAAGCACTCG & ATTCACTGGAGAATACACCTCGC \\
\hline Krt18 & GGAAGTGGATGCCCCCAAAT & TCCTCAATCTGCTGAGACCAGTA \\
\hline Lif & GCGAGATGAGATGCAGGGATT & GGTGGCATTTACAGGGGTGA \\
\hline Met & GCCGGCGGTTTATAAGGTGT & AAGGTACAGCTCTCGTTGCC \\
\hline Rplp0 & TGGGCAAGAACACCATGATG & AGTTTCAGAGCTGGGTTGT \\
\hline
\end{tabular}


Table 11: primers for used for ChIP validation (All belong to mouse species).

\begin{tabular}{|c|c|c|}
\hline Name & Forward primer sequence & Reverse primer sequence \\
\hline Bhlhe40 TSS & GGCTTGAGTCAGACGCGG & TGGTAACGTGGGCGAACC \\
\hline Fam3c distal & TGGATTGACTTTCAAATGCTGGC & AATGAGGCGATTCCTGCTCC \\
\hline $\begin{array}{c}\text { Foxa3 distal } \\
\text { Lif } \\
\text { (ARID1A peak) }\end{array}$ & GGAGAAGCCAGAAACCGCA & AGAAAGGCCCTCAGTCTCCC \\
\hline $\begin{array}{c}\text { Lif } \\
\text { (H3K27ac peak) }\end{array}$ & AAGTTCAGGAAACCAACGGGT & GAGAGACTGTAGCACGGCTG \\
\hline \begin{tabular}{c} 
Met distal \\
\hline
\end{tabular} & AAACCTAAAGCATTTGACCGGC & CCCCCACTACTGTGTTTGATGT \\
\hline
\end{tabular}

\subsubsection{Buffers}

\subsubsection{Buffers used for Western blot.}

Table 12. WCL (whole cell lysis) buffer.

\begin{tabular}{|c|c|c|}
\hline Name & Components & Final concentration \\
\hline \multirow{4}{*}{ WCL buffer } & HEPES $(\mathrm{pH} 7.5)$ & $50 \mathrm{mM}$ \\
\cline { 2 - 3 } & $\mathrm{NaCl}$ & $150 \mathrm{mM}$ \\
\cline { 2 - 3 } & EGTA & $1 \mathrm{mM}$ \\
\cline { 2 - 3 } & Glycine & $10 \%$ \\
\cline { 2 - 3 } & Triton X-100 & $1 \%$ \\
\cline { 2 - 3 } & $\mathrm{NaF}$ & $100 \mathrm{mM}$ \\
\cline { 2 - 3 } & $\mathrm{Na}_{4} \mathrm{P}_{2} \mathrm{O}_{7} \cdot 10 \mathrm{H}_{2} \mathrm{O}$ & $10 \mathrm{mM}$ \\
\hline
\end{tabular}

Table 13. Laemmli buffer (5x).

\begin{tabular}{|c|c|c|}
\hline \multirow{4}{*}{ Name } & Components & Final concentration \\
\hline \multirow{4}{*}{ Laemmli buffer } & Tris (pH6.8) & $225 \mathrm{mM}$ \\
\cline { 2 - 3 } & Glycerin & $50 \%(\mathrm{v} / \mathrm{v})$ \\
\cline { 2 - 3 } & SDS & $5 \%$ \\
\cline { 2 - 3 } & DTT & $100 \mathrm{mM}$ \\
\hline & Bromophenol blue & $0.02 \%$ \\
\hline
\end{tabular}


Table 14. Separating gel buffer and Stacking gel buffer.

\begin{tabular}{|c|c|c|}
\hline Name & Components & Final concentration \\
\hline $\begin{array}{c}\text { Separating gel buffer stock } \\
(\mathrm{pH} 8.8)\end{array}$ & Tris-base & $1.5 \mathrm{M}$ \\
\cline { 2 - 3 } & SDS & $0.4 \%(\mathrm{v} / \mathrm{v})$ \\
\hline $\begin{array}{c}\text { Stacking gel buffer stock } \\
(\mathrm{pH} \mathrm{6.8)}\end{array}$ & Tris-base & $0.5 \mathrm{M}$ \\
\cline { 2 - 3 } & SDS & $0.4 \%(\mathrm{v} / \mathrm{v})$ \\
\hline
\end{tabular}

Table 15. Separating / stacking buffer working solution.

\begin{tabular}{|c|c|c|}
\hline \multirow{4}{*}{ Name } & Components & Volume \\
\hline \multirow{4}{*}{$\begin{array}{c}\text { Separating gel buffer } \\
10 \%\end{array}$} & $\begin{array}{c}\text { Separating gel } \\
\text { buffer stock }\end{array}$ & $20 \mathrm{ml}$ \\
\cline { 2 - 3 } & Acrylamide & $26.6 \mathrm{ml}$ \\
\cline { 2 - 3 } & Glycerol & $4 \mathrm{ml}$ \\
\cline { 2 - 3 } & Aqua dest & $29.3 \mathrm{ml}$ \\
\hline \multirow{4}{*}{$\begin{array}{c}\text { Separating gel buffer } \\
15 \%\end{array}$} & $\begin{array}{c}\text { Separating gel } \\
\text { buffer stock }\end{array}$ & Acrylamide \\
\cline { 2 - 3 } & Glycerol & $40 \mathrm{ml}$ \\
\cline { 2 - 3 } & Aqua dest & $4 \mathrm{ml}$ \\
\cline { 2 - 3 } & & $16 \mathrm{ml}$ \\
\hline
\end{tabular}

Table 16. Running buffer (10x).

\begin{tabular}{|c|c|c|}
\hline Name & Components & Final concentration \\
\hline \multirow{4}{*}{ Running buffer } & Tris-base & $250 \mathrm{mM}$ \\
\cline { 2 - 3 } & Glycerin & $1.92 \mathrm{M}$ \\
\cline { 2 - 3 } & SDS & $1 \%(\mathrm{w} / \mathrm{v})$ \\
\hline
\end{tabular}

Table 17. Transfer buffer (1L).

\begin{tabular}{|c|c|c|}
\hline Name & Components & Volume \\
\hline \multirow{4}{*}{ Transfer buffer } & $5 \times$ Transfer buffer & $200 \mathrm{ml}$ \\
\cline { 2 - 3 } & Ethanol & $200 \mathrm{ml}$ \\
\cline { 2 - 3 } & Aqua dest & $600 \mathrm{ml}$ \\
\hline
\end{tabular}


Table 18. TBS buffer (10x).

\begin{tabular}{|c|c|c|}
\hline Name & Components & Final concentration \\
\hline \multirow{2}{*}{$\begin{array}{c}\text { TBS buffer } \\
(\mathrm{pH} 7.6)\end{array}$} & Tris- $\mathrm{HCl}$ & $0.2 \mathrm{M}$ \\
\cline { 2 - 3 } & $\mathrm{NaCl}$ & 1.37 \\
\hline
\end{tabular}

*Plus $0.1 \%$ Tween-20 before use.

\subsubsection{Buffers for Chromatin Immuno-Precipitation.}

Table 19. Nelson buffer.

\begin{tabular}{|c|c|c|}
\hline Name & Components & Final concentration \\
\hline \multirow{4}{*}{ Nelson buffer } & $\mathrm{NaCl}$ & $150 \mathrm{mM}$ \\
\cline { 2 - 3 } & EDTA $(\mathrm{pH} 8)$ & $20 \mathrm{mM}$ \\
\cline { 2 - 3 } & Tris- $\mathrm{HCl}(\mathrm{pH} \mathrm{7.5)}$ & $50 \mathrm{mM}$ \\
\cline { 2 - 3 } & $\mathrm{NP}-40$ & $0.5 \%$ \\
\cline { 2 - 3 } & Triton X-100 & $1 \%$ \\
\cline { 2 - 3 } & $\mathrm{NaF}$ & $20 \mathrm{mM}$ \\
\hline
\end{tabular}

Table 20. Gomes lysis buffer.

\begin{tabular}{|c|c|c|}
\hline Name & Components & Final concentration \\
\hline \multirow{4}{*}{ Gomes lysis buffer } & $\mathrm{NaCl}$ & $150 \mathrm{mM}$ \\
\cline { 2 - 3 } & EDTA (pH 8.0) & $20 \mathrm{mM}$ \\
\cline { 2 - 3 } & Tris- $\mathrm{HCl}(\mathrm{pH} 8.0)$ & $50 \mathrm{mM}$ \\
\cline { 2 - 3 } & $\mathrm{NP}-40$ & $1 \%$ \\
\cline { 2 - 3 } & Sodium deoxycholate & $0.5 \%$ \\
\cline { 2 - 3 } & $\mathrm{NaF}$ & $20 \mathrm{mM}$ \\
\cline { 2 - 3 } & $\mathrm{SDS}$ & $0.1 \%$ \\
\hline
\end{tabular}


Table 21. Gomes wash buffer.

\begin{tabular}{|c|c|c|}
\hline \multirow{4}{*}{ Name } & Components & Final concentration \\
\hline \multirow{4}{*}{ Gomes wash buffer } & Tris- $\mathrm{HCl}(\mathrm{pH} 8.5)$ & $100 \mathrm{mM}$ \\
\cline { 2 - 3 } & $\mathrm{LiCl}$ & $500 \mathrm{mM}$ \\
\cline { 2 - 3 } & $\mathrm{NP}-40$ & $1 \%(\mathrm{v} / \mathrm{v})$ \\
\cline { 2 - 3 } & Sodium deoxycholate & $1 \%(\mathrm{w} / \mathrm{v})$ \\
\cline { 2 - 3 } & $\mathrm{NaF}$ & $20 \mathrm{mM}$ \\
\cline { 2 - 3 } & EDTA & $20 \mathrm{mM}$ \\
\hline
\end{tabular}

Table 22. Weinmann lysis buffer.

\begin{tabular}{|c|c|c|}
\hline \multirow{2}{*}{ Name } & Components & Final concentration \\
\hline \multirow{3}{*}{ Weinmann lysis buffer } & Tris- $\mathrm{HCl}(\mathrm{pH} 8.0)$ & $50 \mathrm{mM}$ \\
\cline { 2 - 3 } & EDTA & $10 \mathrm{mM}$ \\
\cline { 2 - 3 } & SDS & $1 \%(\mathrm{v} / \mathrm{v})$ \\
\hline
\end{tabular}

\subsubsection{Buffers for chromatin fractionation assay}

Table 23. Buffer A (cytomembrane lysis buffer)

\begin{tabular}{|c|c|c|}
\hline \multirow{4}{*}{ Name } & Components & Final concentration \\
\hline \multirow{4}{*}{$\begin{array}{c}\text { Buffer A } \\
\text { (chromatin fractionation } \\
\text { assay) }\end{array}$} & $\mathrm{KCl}$ & $10 \mathrm{mM}$ \\
\cline { 2 - 3 } & $\mathrm{MgCl}_{2}$ & $10 \mathrm{mM}$ \\
\cline { 2 - 3 } & Triton X-100 & $1.5 \mathrm{mM}$ \\
\cline { 2 - 3 } & DTT & $0.1 \%(\mathrm{v} / \mathrm{v})$ \\
\cline { 2 - 3 } & Sucrose & $1 \mathrm{mM}$ \\
\cline { 2 - 3 } & Glycerol & $0.34 \mathrm{M}$ \\
\cline { 2 - 3 } & Con & $10 \%(\mathrm{v} / \mathrm{v})$ \\
\hline
\end{tabular}

*plus leupeptin $(5 \mu \mathrm{g} / \mathrm{ml})$, pepstatin $(0.5 \mu \mathrm{g} / \mathrm{ml})$ and BPTI $(5 \mu \mathrm{g} / \mathrm{ml})$ before use.

Table 24. Buffer B (nuclei lysis buffer).

\begin{tabular}{|c|c|c|}
\hline \multirow{2}{*}{ Name } & Components & Final concentration \\
\hline \multirow{3}{*}{ Buffer B } & EDTA $(\mathrm{pH} 8)$ & $3 \mathrm{mM}$ \\
\cline { 2 - 3 } & EGTA $(\mathrm{pH} 8)$ & $0.2 \mathrm{mM}$ \\
\cline { 2 - 3 } & DTT & $1 \mathrm{mM}$ \\
\hline
\end{tabular}

*plus leupeptin $(5 \mu \mathrm{g} / \mathrm{ml})$, pepstatin $(0.5 \mu \mathrm{g} / \mathrm{ml})$ and BPTI $(5 \mu \mathrm{g} / \mathrm{ml})$ before use. 


\subsubsection{ATAC buffer}

Table 25. ATAC lysis buffer.

\begin{tabular}{|c|c|c|}
\hline \multirow{2}{*}{ Name } & Components & Final concentration \\
\hline \multirow{3}{*}{ ATAC lysis buffer } & Tris- $\mathrm{HCl}(\mathrm{pH} 7.4)$ & $10 \mathrm{mM}$ \\
\cline { 2 - 3 } & $\mathrm{NaCl}$ & $10 \mathrm{mM}$ \\
\cline { 2 - 3 } & $\mathrm{MgCl}_{2}$ & $3 \mathrm{mM}$ \\
\cline { 2 - 3 } & $\mathrm{NP}-40$ & $0.1 \%(\mathrm{v} / \mathrm{v})$ \\
\hline
\end{tabular}

\subsubsection{Cell culture}

Table 26. Cell culture components.

\begin{tabular}{|c|c|c|}
\hline Components & $\begin{array}{c}\text { Catalogue } \\
\text { Number }\end{array}$ & Company \\
\hline $\begin{array}{c}\text { Dulbecco's Modified Eagle Medium } \\
\text { (DMEM) }\end{array}$ & 41965-039 & Life Technologies Corp., Paisley, UK \\
\hline Fetal calf serum (FCS) & $10270-106$ & Life Technologies Corp., Paisley, UK \\
\hline Penicillin-streptomycin & P0781 & Sigma-Aldrich, St. Louis, USA \\
\hline $\begin{array}{l}\text { Hank's Balanced Salt Solution } \\
\qquad(\text { HBSS })\end{array}$ & 14175095 & Life Technologies Corp., Paisley, UK \\
\hline Waymouth's Medium & 31220023 & Life Technologies Corp., Paisley, UK \\
\hline McCoy's 5A (Modified) Medium & 26600023 & Life Technologies Corp., Paisley, UK \\
\hline Soybean trypsin inhibitor (SBTI) & T6522 & Sigma-Aldrich, St. Louis, USA \\
\hline Collagen I, rat tail & ALX-522-435 & $\begin{array}{c}\text { Enzo Life Sciences GmbH, } \\
\text { Loerrach, Germany }\end{array}$ \\
\hline Collagenase VIII & C2139 & Sigma-Aldrich, St. Louis, USA \\
\hline Collagenase P & 11213857001 & Sigma-Aldrich, St. Louis, USA \\
\hline Nicotinamide & N0636 & Sigma-Aldrich, St. Louis, USA \\
\hline Bovine pituitary extract & P1476 & Sigma-Aldrich, St. Louis, USA \\
\hline $\begin{array}{l}\text { Insulin-Transferrin-Selenium- } \\
\text { Ethanolamine (ITS-X) 100x }\end{array}$ & 51500056 & $\begin{array}{c}\text { Invitrogen } \mathrm{GmbH} \text {, Karlsruhe, } \\
\text { Germany }\end{array}$ \\
\hline
\end{tabular}




\subsubsection{Solutions for acinar-epithelial explants in 3D culture}

Table 27. Solution 1 (Washing solution).

\begin{tabular}{|c|c|c|}
\hline \multirow{2}{*}{ Name } & Components & Final concentration \\
\hline \multirow{3}{*}{ Solution 1 } & McCoy's Medium & - \\
\cline { 2 - 3 } & $10 \% \mathrm{BSA}$ & $0.1 \%$ \\
\cline { 2 - 3 } & SBTI $(10 \mathrm{mg} / \mathrm{ml})$ & $0.2 \mathrm{mg} / \mathrm{ml}$ \\
\hline
\end{tabular}

Table 28. Solution 2 (Digestion solution).

\begin{tabular}{|c|c|c|}
\hline \multirow{2}{*}{ Name } & Components & Final concentration \\
\hline \multirow{3}{*}{ Solution 2 } & McCoy's Medium & - \\
\cline { 2 - 3 } & $10 \% \mathrm{BSA}$ & $0.1 \%$ \\
\cline { 2 - 3 } & SBTI $(10 \mathrm{mg} / \mathrm{ml})$ & $0.2 \mathrm{mg} / \mathrm{ml}$ \\
\cline { 2 - 3 } & Collagenase VIII & $1.2 \mathrm{mg} / \mathrm{ml}$ \\
\hline
\end{tabular}

Table 29. Solution 3 (Culture solution).

\begin{tabular}{|c|c|c|}
\hline Name & Components & Final concentration \\
\hline \multirow{8}{*}{ Solution 3} & Waymouth's Medium & - \\
\hline & $10 \%$ BSA & $0.1 \%$ \\
\hline & SBTI (10 mg/ml) & $0.2 \mathrm{mg} / \mathrm{ml}$ \\
\hline & Pen / Strep & $1 \%(\mathrm{v} / \mathrm{v})$ \\
\hline & BPE (14 mg/ml) & $50 \mu \mathrm{g} / \mathrm{ml}$ \\
\hline & $\begin{array}{c}\text { Insulin-Transferrin- } \\
\text { Selenium-Ethanolamine }\end{array}$ & $1 \%$ \\
\hline & Nicotinamide (20mM) & $200 \mu \mathrm{M}$ \\
\hline & FCS & $30 \%$ or $0.1 \%$ \\
\hline
\end{tabular}

Table 30. Collagen solution.

\begin{tabular}{|c|c|c|}
\hline \multirow{2}{*}{ Name } & Components & Volume ( per 1 ml solution) \\
\hline \multirow{4}{*}{ Collagen } & $10 x$ PBS & $0.1 \mathrm{ml}$ \\
\cline { 2 - 3 } & Sterile $1 \mathrm{~N} \mathrm{NaOH}$ & $0.0115 \mathrm{ml}$ \\
\cline { 2 - 3 } & Collagen $(5 \mathrm{mg} / \mathrm{ml})$ & $0.5 \mathrm{ml}$ \\
\cline { 2 - 3 } & Sterile $\mathrm{dH}_{2} \mathrm{O}$ & $0.3885 \mathrm{ml}$ \\
\hline
\end{tabular}




\subsection{Methods}

\subsubsection{Animal studies}

\subsubsection{Mouse lines}

Control cohorts: $p 48$-Cre mice

Experimental cohorts: Arid1 $a^{f / /+} ; p 48-C r e$ and Arid1 $a^{f / / f t} ; p 48-C r e$ mice.

All animal experiments were approved by the Institutional Animal Care and Use Committee at University Medical Center Goettingen and performed in accordance with the related ethical regulations (No. 33.9-42502-04-14/1634 and 33.9-42502-04-19/3085).

The establishment of the p48-Cre mice has been previously described (Nakhai et al., 2007). The Arid1 $a^{f / f l}$ mouse was a kind gift from Prof. Steven Johnsen and was crossed with $p 48$-Cre mouse to generate the experimental cohorts harboring pancreatic-acinarspecific Arid1a depletion. All the mice are with mixed background. Arid1a ${ }^{f /+} ; p 48-C r e$ and Arid1a $a^{f / f f} ; p 48-C r e$ mice were born according to the predicted Mendelian ratio and with a balanced sex composition. Genotyping was performed by PCR using genomic DNA extracted from mouse tail biopsy. Mice were assigned to time-point- (2/3/6/12/18 months) or survival- analysis after genotyping. Survival data were recorded when mice reached end-point criteria, such as impuissance, lack of social interaction and lethargy.

\subsubsection{Tissue harvesting}

Mice tissues were harvested at the indicated time points and from the end-point-reaching survival mice. Pancreas, spleen and liver specimens were collected. Pancreas was separated into several portions and the representative parts (from pancreas head and tail) were kept overnight (together with liver and spleen) in $4 \%$ formaldehyde solution prior to the paraffin embedding. The rest of the pancreas was kept in cryo pure vials, snap frozen and stored at $-80^{\circ} \mathrm{C}$ for protein and RNA isolation.

\subsubsection{Paraffin embedding and tissue sectioning}

Formaldehyde-fixed tissue was processed with dehydration by Leica Biosystems tissue processor. The protocol was used as described below: Formalin for $75 \mathrm{~min}, 55 \%$ Ethanol for $30 \mathrm{~min}, 85 \%$ Ethanol for $45 \mathrm{~min}, 96 \%$ Ethanol for $60 \mathrm{~min}, 99 \%$ Ethanol for $75 \mathrm{~min}$, 
99 \% Ethanol for $70 \mathrm{~min}, 99 \%$ Ethanol for $90 \mathrm{~min}$, xylol for $20 \mathrm{~min}$, xylol for $30 \mathrm{~min}$, xylol for $70 \mathrm{~min}$, paraffin for $30 \mathrm{~min}$, paraffin for $45 \mathrm{~min}$, paraffin for $90 \mathrm{~min}$. Tissues were embedded in paraffin with metal mold, sectioned into $4 \mu \mathrm{m}$ sections using a microtome and kept at $37^{\circ} \mathrm{C}$ overnight prior to staining.

\subsubsection{Hematoxylin and eosin (H\&E) staining}

Paraffin-embedded mice tissues were deparaffinized by Roticlear solution (2x $10 \mathrm{~min}$ ) and rehydrated by the ethanol series (99\%, $99 \%, 96 \%, 80 \%, 70 \%, 50 \%$, each 3 min). The slides were washed once in tap water and placed in hematoxylin solution for 6 min, followed by keeping in running tap water for $7 \mathrm{~min}$ to remove the superficial staining. The slides were then transferred to eosin solution (diluted in 0.2 \% acetic acid) for 10-15 seconds to stain the cytoplasm, dehydrated in increasing concentration of ethanol $(70 \%$, $80 \%, 96 \%, 99 \%$ ) and cleared in Roticlear. Finally, the slides were mounted using Rotimount and ready for microscopic examination.

\subsubsection{Alcian blue staining}

Deparaffinization and rehydration steps were the same as H\&E staining. Slides were stained in alcian blue $8 G X$ solution for $30 \mathrm{~min}$, followed by a short rinse in $3 \%$ acetic acid (in which alcian blue dye is dissolved). The slides were further incubated in nuclear fast red solution for $5 \mathrm{~min}$ to counterstain nuclei and washed in running tap water for 1 min. Further, the sections were dehydrated and mounted. For ADM/PanIN quantification, images were taken under $4 x$ (eye-piece magnification). The region of ADM or PanIN lesions, based on alcian blue staining positivity and morphology, was selected with 'Polygon selections' tool and measured by 'Analyze-Measure' function. The size of the entire pancreas in each field of view was also measured simultaneously and finally the ADM/PanIN to pancreas ratio was calculated. Statistical analysis was performed using two-tailed Student's unpaired $t$-test by Prism 8.

\subsubsection{Immunohistochemistry (IHC) and quantification}

Paraffin-embedded mice tissues were de-waxed and rehydrated with distill water. Antigen-retrieving was achieved by boiling slides in a microwave in Citrate buffer ( $\mathrm{pH} 6.0)$ or TE buffer followed by sub-boiling for $6-15 \mathrm{~min}$. Boiled slides were kept inside the microwave for another $10 \mathrm{~min}$, cooling down in a $4{ }^{\circ} \mathrm{C}$ freezer to approximate body- 
temperature (around $30 \mathrm{~min}$ ). To block the endogenous peroxidase, slides were washed three times in tap water and incubated $10 \mathrm{~min}$ in $3 \%$ hydrogen peroxide which was freshly diluted from $30 \%$ stock solution. Further, the slides were washed in tap water and aligned into Shandon Sequenza ${ }^{\mathrm{TM}}$ rack (cadenza system) with PBS containing $0.1 \%$ Tween-20 (PBST). After washing the slides with PBST for three times to ensure that slides were completely rinsed, the blocking solution $10 \% \mathrm{BSA}(\mathrm{w} / \mathrm{v})$ was applied to the slides through cadenza system and allowed to stay for $1 \mathrm{~h}$ at room temperature to avoid the unspecific antigen-antibody reaction. Primary antibodies (listed in Table 5) were diluted in PBST and were applied as $200 \mu \mathrm{l} / \mathrm{slide}$ and incubated overnight at $4{ }^{\circ} \mathrm{C}$. The next day, three rounds of PBST were used to wash the slides prior to the incubation of the secondary antibody. Secondary antibodies were in 1:200 dilutions and kept on slides for $1 \mathrm{~h}$ at room temperature, followed by 3 times of washing by PBST. Further, avidinbiotin complex ( $\mathrm{AB}$ complex) was prepared $30 \mathrm{~min}$ prior to adding to the slides and incubated for $1 \mathrm{~h}$ at room temperature. Slides were unassembled from cadenza system and the signal of the primary antibody was detected using DAB peroxidase substrate as $150 \mu \mathrm{l} / \mathrm{slide}$ and waited for corresponding time span for adequate reaction. Finally, slides were stained with hematoxylin to label nuclei, dehydrated, mounted as the same as described in H\&E protocol.

For IHC using antibodies raised in mouse species, M.O.M kit was used following manufacturer's instruction. M.O.M mouse lgG blocking reagent was used for blocking. M.O.M diluent solution was used to dilute primary and secondary antibodies and was added to the slides $5 \mathrm{~min}$ prior to the overnight primary antibody incubation. The rest of the steps are the same as the normal IHC procedure.

Images were captured with a microscope-attached Olympus SC180 using Cellsens Entry software. Quantifications of IHC and ADM/PanIN regions were performed using ImageJ from 5 mice per condition. Briefly, for IHC quantification, 10x (eye-piece magnification) images were taken of the entire pancreas area on each slide. Images were uploaded into ImageJ and counted under 'Color Threshold' by adjusting RGB color mode to ensure all the positive staining are marked by red particles. The percentage of area covered the positive particles were calculated by 'Analyze particle' function of ImageJ. Statistical analysis was performed using two-tailed Student's unpaired $t$-test by Prism 8 . 


\subsubsection{Cell culture}

\subsubsection{Cell culture conditions for adherent cells}

266-6 murine pancreatic acinar cell line was established as described before (Korc et al., 1992). Cells were cultured in Dulbecco's modified Eagle's Medium containing $4.5 \mathrm{~g} / \mathrm{L} \mathrm{D}$ Glucose, L- Glutamine supplemented with $10 \%$ fetal calf serum (FCS) at $37^{\circ} \mathrm{C}$ incubator with $5 \%$ carbon dioxide supply.

To make cell stocks, cells were washed with PBS, trypsinized and pelleted. Cell pellet was resuspended in freezing medium which consists of $90 \%$ FCS and $10 \%$ DMSO and aliquoted as $2^{*} 10^{6}$ cells/ $1 \mathrm{ml}$ freezing media/ cryovial and stored in Mr. Frosty ${ }^{\mathrm{TM}}$ in -80 ${ }^{\circ} \mathrm{C}$ freezer overnight before transferring to liquid nitrogen for long-term storage.

When thawing cells for experiment, cell stocks were rapidly defrosted in $37^{\circ} \mathrm{C}$ water bath. The content was transferred into a $15 \mathrm{ml}$ falcon containing $4 \mathrm{ml}$ pre-warmed fresh culture medium and centrifuged at $1200 \mathrm{rpm}, 3 \mathrm{~min}$. The cell pellet was slowly diluted in $9 \mathrm{ml}$ culture media and transferred to a cell culture flask with appropriate size to ensure the high density of cell resuspension. Cells were passaged at least once before being used for any experiment.

\subsubsection{Transient transfection}

\subsubsection{Transfection with small interfering RNA (siRNA) targeting Arid1a}

siArid1a was freshly prepared by mixing ON-TARGETplus Arid1a siRNA \#7 and \#8 (20 $\mu \mathrm{M}$ stock) in the proportion of 1:1. For each well in 6-well plate, lipid-siRNA complexes were prepared by adding $200 \mu$ l Opti-MEM ${ }^{\circledR}$ medium containing $6 \mu$ l siLentFect lipid reagent and $360 \mathrm{nM}$ siArid1a or non-targeting siRNA, pipetted to mix and kept at room temperature for $15 \mathrm{~min}$. Simultaneously, cells were trypsinized and seeded as $1.2 * 10^{6}$ cells in $1 \mathrm{ml}$ medium/well. Lipid-siRNA complexes were added to the cell-suspension to obtain $60 \mathrm{nM}$ siArid1a as the final concentration.

\subsubsection{Transfection with plasmid constructs}

For overexpression of NFATc1, cells were seeded in $60 \mathrm{~mm}$ dishes and grown into $60 \%$ confluency and transfected with $8 \mu \mathrm{g}$ NFATc1 constitutive active construct (kindly provided by N.A. Clipstone) by using Lipofectamine ${ }^{\circledR} 2000$ regent diluted in Opti-MEM ${ }^{\circledR}$ 
medium. The volume for each component within transfection system was following manufacturer's instruction of Lipofectamine ${ }^{\circledR} 2000$ regent.

\subsubsection{Epidermal growth factor (EGF) and Cyclosporine A (CsA) treatment}

The preparation of the EGF stock solution was following manufacturer's instruction. 0.2 mg EGF powder was dissolved in $200 \mu \mathrm{l} 10 \mathrm{mM}$ acetic acid to $1 \mathrm{mg} / \mathrm{ml}$. To further dilute to $10 \mu \mathrm{g} / \mathrm{ml}, 19.8 \mathrm{ml} 0.1 \%$ BSA was added, followed by sterilizing through $0.2 \mu \mathrm{m}$ strainer. Prior to EGF treatment, 266-6 cells in culture were washed with PBS for two times followed by starving in serum-free DMEM medium overnight $(\sim 14 \mathrm{~h})$. The next day, starved cells were treated with $40 \mathrm{ng} / \mathrm{ml}$ EGF for 8 hours and harvested for corresponding experiments. The control cells were treated with $0.1 \% \mathrm{BSA}$.

To block calcineurin activity in order to prevent NFATc1 translocation into the nucleus, cells were treated with $1 \mu \mathrm{M}$ CsA while control groups were treated with absolute ethanol for $24 \mathrm{~h}$. RNA or lysates were harvested for expression analysis.

\subsubsection{Reporter assay}

266-6 cells were seeded in 24-well plate at the density of $1.5 * 10^{5}$ cells/well and transfected concurrently with Arid1a siRNA (the transfection system was prepared by scaling down each components described in method transient transfection part). Culture media was exchanged to normal 10\% FCS containing DMEM $10 \mathrm{~h}$ post-transfection. On the following day, all the wells were transfected with $100 \mu$ l Opti-MEM ${ }^{\circledR}$ containing $2 \mu \mathrm{l}$ Lipofectamine ${ }^{\circledR} 2000$, pNFATc1-luc construct (500 ng, Stratagene) and Renilla-luciferase vector (15 ng, kindly provided by R. Urrutia). Control and EGFR-overexpression wells were mixed with $500 \mathrm{ng}$ control vector pMCV (Stratagene) and pSG5-v-ErbB-EGFR construct (kindly provided by M. Privalsky), respectively. $24 \mathrm{~h}$ later, the media was taken out and replaced with $1 x$ passive lysis buffer which was diluted from $5 x$ by $\mathrm{H}_{2} \mathrm{O}$. After incubating on a shaker (200-600 rpm) for $15 \mathrm{~min}$ at RT, $30 \mu \mathrm{l}$ cell lysate was transferred into a white 96-well plate with a flat bottom (to avoid the inter-well signal contamination during measuring), mixed with $30 \mu \mathrm{l}$ 'Luciferase Substrate' (which was prepared according to the Promega Dual Luciferase Reporter Assay Kit instruction) and measured the first luminescent signal by luminometer. Further, $30 \mu$ freshly prepared 'Stop \& Glo ${ }^{\circledR}$ ' reagent (substrate + buffer) was added to quench the firefly reaction and the second measurement for Renilla luminescent was initiated. The instrument setup used for 
luminometer measurement was: enable shaking (low 3 ) followed by a 0.5 -second activity read time.

\subsubsection{Acinar cell isolation and in vitro culture}

The pancreas of 8-week-old $p 48-C r e$, Arid $1 a^{f /+} ; p 48-C r e$ and Arid1 $a^{f / f t} ; p 48-C r e$ mice were harvested in ice-cold HBSS, dissociated by surgical scissors in a $10 \mathrm{~cm}$ cell culture dish filled with $5 \mathrm{ml}$ cold HBSS and centrifuged at low speed (300 rpm, $5 \mathrm{~min}$ ) to remove blood vessels and adipose tissue. The remaining explant was digested in $5 \mathrm{ml}$ solution 2 at 37 ${ }^{\circ} \mathrm{C}$ for $20 \mathrm{~min}$, pipetted every $2 \mathrm{~min}$ using a $10 \mathrm{ml}$ pipette. The tissue-containing tube was washed with $10 \mathrm{ml}$ solution 1 and centrifuged (300 rpm, $5 \mathrm{~min}$ ) before adding a second time of solution 2 and cultivated at $37^{\circ} \mathrm{C}$ for $10 \mathrm{~min}$. Next, tissue suspension was transferred into a new $50 \mathrm{ml}$ tube by filtering through a $100 \mu \mathrm{m}$ cell strainer and the tissue fragments were ground using a syringe plunger. The remaining cells were collected by washing the mesh with two times of $10 \mathrm{ml}$ solution 1 and pelleted. Recovering was achieved by easing the acinar cells in $5 \mathrm{ml}$ solution 3 containing 10\% FCS for 1 hour at $37^{\circ} \mathrm{C}$. Meanwhile, 12-well plates were coated with collagen $(500 \mu \mathrm{l} /$ well) and placed in incubator for 1 hour to solidify. Recovered acinar cells were pelleted down and counted, an equivalent number of cells were re-suspended in $100 \mu \mathrm{l} 0.1 \%$ FCS-containing solution 3 and then mixed with $100 \mu \mathrm{l}$ collagen solution and equally distributed by a $1000 \mu \mathrm{l}$ pipette tip into 12-well plate. Further, EGF or control vehicle diluted in $300 \mu \mathrm{l} 0.1 \%$ FCScontaining solution 3 was added on top until the acinar membrane was formed (approx. $45 \mathrm{~min}$ ). EGF-containing media was changed freshly every second day. Images were taken at $4^{\text {th }}-5^{\text {th }}$ day after plating under $40 x$ magnification from duplicate wells by light microscopy. The number of all the visible ADMs found in each well was recorded and plotted by Prism 8 and RNA was isolated subsequently.

For acinar cell transformation assay performed with 266-6 cells, cells were transfected with siArid1a in $60 \mathrm{~mm}$ cell culture dish and allowed to attach for 24 hours. Cells were split and seeded into 12-well plate with the density of $5 * 10^{5}$ cells/well and subjected to the equivalent procedure of acinar-explant culture as described above. 


\subsubsection{Molecular biology}

\subsubsection{RNA extraction from $266-6$ cells}

Cell culture media was aspirated and $500 \mu \mathrm{l}$ TRIzol was added to the cells. Cells were scraped and collected in a $1.5 \mathrm{ml}$ Eppendorf tube and further added with $200 \mu \mathrm{l}$ chloroform, vortexed and centrifuged at $13500 \mathrm{rpm}$ for $15 \mathrm{~min}\left(4^{\circ} \mathrm{C}\right)$. The upper aqueous phase was mixed with $500 \mu \mathrm{l}$ isopropanol and incubated at room temperature for $10 \mathrm{~min}$ allowing RNA to precipitate, followed by centrifugation at $13500 \mathrm{rpm}, 30 \mathrm{~min}\left(4^{\circ} \mathrm{C}\right)$. After being washed twice in $1 \mathrm{ml} 75 \% \mathrm{EtOH}$, the pellet was dried and ultimately dissolved in $20-50 \mu \mathrm{l}$ nuclease-free water and kept in $-80^{\circ} \mathrm{C}$. The concentration and RNA quality were measured using a Nano-photometer P-330.

\subsubsection{RNA extraction from acinar explants}

The top medium was collected in a $1.5 \mathrm{ml}$ tube to harvest cells in the medium by centrifugation (1200 rpm, $3 \mathrm{~min}, \mathrm{RT}$ ). The collagen-cells disc was washed carefully with $300 \mu \mathrm{l} \mathrm{HBSS}$. Collagenase $\mathrm{P}(10 \mathrm{mg} / \mathrm{ml})$ was further diluted by HBSS into 1:50 and added in the wells $(500 \mu \mathrm{l} /$ well $)$ to degrade collagen. The collagen-cells disc was transferred together with the collagenase $P$ into a new $1.5 \mathrm{ml}$ Eppi using a $1 \mathrm{ml}$ cut tip and incubated on a $37^{\circ} \mathrm{C}$ Thermo Block for 10-15 min, pipetting up and down using $1 \mathrm{ml}$ cut tips until no collagen clumps remained. The mixture was centrifuged at $4000 \mathrm{rpm}$ for $2 \min \left(4^{\circ} \mathrm{C}\right)$, the supernatant was removed and the cell pellet was washed with $300 \mu \mathrm{l}$ ice-cold HBSS to get rid of the remaining collagen. The final cell pellet was pooled together into the cells collected from the top medium and resuspended in TRIzol and proceeded with RNA extraction as described above.

\subsubsection{3 cDNA synthesis}

$1 \mu \mathrm{g}$ RNA was reversely transcribed into cDNA according to the manufacturer's instruction provided by iScript cDNA Synthesis Kit. Transcription was initiated by incubating the RNA-transcriptase system at room temperature for $5 \mathrm{~min}$ and $46{ }^{\circ} \mathrm{C}$ for $20 \mathrm{~min}$. Enzymatic reaction was stopped by heating the samples at $95^{\circ} \mathrm{C}$ for $1 \mathrm{~min}$. The cDNA was further diluted to $10 \mathrm{ng} / \mu \mathrm{l}$. 


\subsubsection{Quantitative real-time polymerase chain reaction (qPCR)}

qPCR reaction system was prepared as below:

Table 31. qPCR reaction components

\begin{tabular}{|l|l|}
\hline Components & Volume per reaction $(\mathbf{1 0} \boldsymbol{\mu l})$ \\
\hline SYBR green mix & $5 \mu \mathrm{l}$ \\
\hline $\mathrm{H}_{2} \mathrm{O}$ & $3.9 \mu \mathrm{l}$ \\
\hline Forward primer & $0.05 \mu \mathrm{l}$ \\
\hline Reverse primer & $0.05 \mu \mathrm{l}$ \\
\hline cDNA & $1 \mu \mathrm{l}$ \\
\hline
\end{tabular}

All the reactions were set in triplicates and conducted by StepOnePlus Real-Time PCR System with the following program:

Table 32. StepOnePlus Real-Time PCR program.

\begin{tabular}{|c|c|c|}
\hline $95^{\circ} \mathrm{C}$ & $20 s$ & Initiating phase \\
\hline $95^{\circ} \mathrm{C}$ & $3 s$ & \multirow{2}{*}{$\begin{array}{l}40 \text { cycles (mRNA qPCR) or } \\
55 \text { cycles (ChIP-qPCR) }\end{array}$} \\
\hline $60^{\circ} \mathrm{C}$ & $30 \mathrm{~s}$ & \\
\hline $95^{\circ} \mathrm{C}$ & $15 s$ & \multirow{3}{*}{ Melt curve phase } \\
\hline $60^{\circ} \mathrm{C}$ & $1 \mathrm{~min}$ & \\
\hline $95^{\circ} \mathrm{C}$ & $15 \mathrm{~s}$ & \\
\hline
\end{tabular}

\subsubsection{Chromatin immunoprecipitation}

266-6 cells were grown in $15 \mathrm{~cm}$ dishes to $85 \%$ confluency and treated with EGF (40 $\mathrm{ng} / \mathrm{ml}$ ) or control vehicle $0.1 \%$ BSA for $8 \mathrm{~h}$. For ARID1A ChIP, cells were crosslinked primarily for $20 \mathrm{~min}$ in $15 \mathrm{mM}$ ethylene glyco-bis (succinimidyl succinate) (EGS), $20 \mathrm{~min}$ in 2 mM EGS, followed by $1 \%$ PFA fixation for 40 min at $4{ }^{\circ} \mathrm{C}$ on a shaker. For NFATc1 ChIP, cells were crosslinked in $2 \mathrm{mM}$ EGS for $20 \mathrm{~min}$ at RT followed by 20 min fixation in 1\% PFA for another $20 \mathrm{~min}$ at RT. The subsequent procedure was conducted using ChIP-IT High Sensitivity Kit (ACTIVE-MOTIF) according to the manufacturer's instructions.

For H3K27ac ChIP, 1\% formaldehyde was added to the cells for 15-20 min and quenched by $125 \mathrm{mM}$ Glycine. Nucleus was released and pelleted in modified Nelson buffer 
(Nelson 2006) and lysed in 500-750 $\mu$ l Gomes lysis buffer (Gomes 2006) supplemented with protease and phosphatase inhibitors. The lysates were aliquoted into sonication tubes and sheared by a Diagenode Biorupter-Pico (30 s On/ 30 s Off). The efficiency of the shearing was confirmed by agarose gel electrophoresis and the appropriatelysheared chromatin was pre-cleared by incubating with $100 \mu \mathrm{l} 50 \%$ slurry Protein A/G Agarose beads at $4^{\circ} \mathrm{C}$ for $1 \mathrm{~h}$ followed by centrifugation at $12000 \times g$ for $2 \min \left(4^{\circ} \mathrm{C}\right)$. The supernatant was aliquoted into IgG, IP and incubated with the indicated primary antibody overnight and meanwhile one tube was saved as Input and kept in $-80^{\circ} \mathrm{C}$. Agarose beads were blocked in $1 \mu \mathrm{g} / \mu \mathrm{l}$ BSA diluted in Gomes lysis buffer simultaneously. The next day, blocked beads were added to the samples to bind on antibody-protein complexes and immune complexes were washed once in Gomes lysis buffer, twice with Gomes wash buffer twice in Gomes lysis buffer and twice in TE buffer for removal of the un-specific chromatin interactions with the agarose beads. For DNA reverse-crosslinking and precipitation, samples (include input) were incubated with $10 \mu \mathrm{g}$ RNase A for $30 \mathrm{~min}$ $\left(37^{\circ} \mathrm{C}\right)$ followed by protein degradation by proteinase $\mathrm{K}\left(65^{\circ} \mathrm{C}\right.$, overnight). DNA was eluted by resuspending beads in $100 \mu \mathrm{l}$ Tris- $\mathrm{HCl}(\mathrm{pH} 8)$ at $65^{\circ} \mathrm{C}$ for $10 \mathrm{~min}$ and extracted by Phenol/Chloroform/Isoamylic alcohol. Final DNA was re-suspended in 30-40 $\mu$ l distilled $\mathrm{H}_{2} \mathrm{O}$ and used for high-throughput sequencing or qRT-PCR.

\subsubsection{Protein biochemistry}

\subsubsection{Protein isolation from cells}

Cultured cells were washed twice with cool PBS and lysed according to diverse purpose. To prepare whole cell lysates, cells were scraped and lysed in WCL buffer for 30 min on ice, followed by centrifugation at $13500 \mathrm{rpm}$ for $20 \mathrm{~min}$. To prepare different cellular extracts, cells were incubated in Buffer A (for chromatin fractionation assay) and kept on ice for $5 \mathrm{~min}$. Nuclei were pelleted by low speed centrifugation $\left(1300 \times \mathrm{g}, 5 \mathrm{~min}, 4^{\circ} \mathrm{C}\right)$ and the supernatant was collected as cytoplasmic fraction with one further clarification centrifugation at high speed $\left(20,000 \times \mathrm{g}, 15 \mathrm{~min}, 4^{\circ} \mathrm{C}\right)$ to remove cell debris and insoluble aggregates. The nuclei pellet was resuspended in Buffer $A^{*}$ supplemented with $1 \mathrm{mM}$ $\mathrm{CaCl}_{2}$ and 0.2 unit Micrococcal nuclease/reaction. After incubating at $37^{\circ} \mathrm{C}$ for $1 \mathrm{~min}$, $1 \mathrm{mM}$ EGTA was added to quench the reaction. Nuclei was collected by centrifugation $\left(1300 \times \mathrm{g}, 4 \mathrm{~min}, 4^{\circ} \mathrm{C}\right)$ and lysed in Buffer $\mathrm{B}$ for $30 \mathrm{~min}$ on ice. Afterwards, nuclear soluble extracts were collected by centrifugation $\left(1700 \times g, 4 \mathrm{~min}, 4^{\circ} \mathrm{C}\right)$ and the remaining 
chromatin pellet was dissolved in 1x Laemmli buffer and sheared by sonication (12s On, 30 s Off, 6 cycles) to collect the chromatin-bound protein. All the lysates were subjected to western blotting analysis.

\subsubsection{Bradford protein determination assay}

Bradford reagent containing Coomassie Blue G-250 was used to determine the protein concentration. Assay was performed in duplicates in the clear 96-well plate with flat bottom. Bovine Serum Albumin (BSA) standard curve was set up by adding $1 \mu \mathrm{g} / 2 \mu \mathrm{g} / 4$ $\mu \mathrm{g} / 6 \mu \mathrm{g}$ BSA (which was diluted in the corresponding buffer in the lysate preparation) into $200 \mu \mathrm{l}$ Bradford reagent. $1 \mu \mathrm{l}$ of each protein sample was processed in the same way and result was measured by a spectrophotometer at $595 \mathrm{~nm}$ absorbance.

\subsubsection{Western blot analysis}

Protein was separated by sodium dodecyl sulfate-polyacrylamide gel electrophoresis (SDS-PAGE) based on their molecular weight. $30 \mu \mathrm{g}$ protein was denatured in Laemmli buffer, heated at $95^{\circ} \mathrm{C}$ for $7 \mathrm{~min}$ and spun down briefly. Immuno-blotting was performed by loading the protein samples onto SDS-PAGE gel and blotting the gel on a $0.45 \mu \mathrm{m}$ nitrocellulose membrane using Trans- Blot Turbo transfer system. Protein bands were visualized using Ponceau S solution and cut according to the indicative protein marker. Membranes were blocked in 5\% milk in PBS-Tween at room temperature for 1-2 $\mathrm{h}$ prior to the overnight primary antibody incubation. The next day, membranes were washed three times in PBS-T (5 min $\times 3$ ) and incubated with peroxidase-conjugated secondary antibodies 1 hour at RT. After three times washing by PBS-T, protein bands were visualized by an Intas ECL Chemocam Imager with the help of chemiluminescent substrate ECL reagent. The primary and secondary-antibodies used in this study were listed in Table 6. 


\subsubsection{Next-generation sequencing}

\subsubsection{ChIP-seq library preparation and data analysis}

ChIP-seq samples were prepared in duplicates per condition. Library for ChIP-seq was prepared using Microplex Library Preparation V2 following the manufacturer's instruction. $0.5 \mathrm{ng}$ double-stranded ChIP-DNA was utilized as start material, repaired into blunt ends and subsequently ligated with stem-loop adaptors. Libraries were amplified and sizeselected by AMPure beads. Library quantification was performed using Agilent Bioanalyzer 2100 . Finally, library was prepared as $2 \mathrm{nmol} / \mathrm{L}$ for sequencing.

ARID1A and H3K27ac ChIP were sequenced on a HiSeq 4000 by NGS Integrative of Genomics Core (NIG), UMG. Analysis of the sequencing data was performed on the public server of usegalaxy.org (Afgan et al., 2018). The reads quality was evaluated by fastQC (Version 0.72; Andrews, 2010). Single-end 50bp reads were aligned to mouse genome MGSCv37/mm9 by Bowtie2 (Version 2.3.4.2; Langmead et al., 2009; Langmead and Salzberg, 2012). Low-quality reads (MAPQ < 5) and PCR duplicates were filtered out using Samtools (Li et al., 2009; Li, 2011) to avoid the interference. Peaks were called by MACS2 (Version 2.1.1.20160309.0; Zhang et al., 2008; Feng et al., 2012) with the criteria as fold enrichment $>5$ compared to background and FDR $<0.05$. For H3K27ac ChIP, the adjacent regions with FDR $<0.1$ were composited as broad peaks. Transcription start site (TSS)-centered $1 \mathrm{~kb}$ regions were considered as promoters while H3K27ac peaks with the exclusion of $1 \mathrm{~kb}$ of an annotated TSS were defined as active enhancers. Differential binding analysis was performed to identify the peaks that were respectively enriched in each condition using $\mathrm{R}$ Bioconductor package DiffBind (Stark et al., 2011; Ross-Innes et al., 2012). H3K27ac peaks were annotated by Genomic Regions Enrichment Annotation Tool (GREAT 3.0) (http://great.stanford.edu/public/html/) following the basal plus extension association rule: $1 \mathrm{~kb}$ upstream, $1 \mathrm{~kb}$ downstream, plus $1000 \mathrm{~kb}$ extension and ARID1A peaks were annotated by GREAT with the single nearest gene association rule with the default setting.

The HOMER findMotifsGenome.p/ script was utilized to search for de novo motifs.

\subsubsection{ATAC-seq library preparation and data analysis}

ATAC-seq library preparation was conducted using Nextera DNA Library Prep (Illumina) 
as previously described (Buenrostro et al., 2013). Primary acinar cells of $p 48-C r e$ and Arid1 $a^{f / f t} ;$ p48-Cre mice were isolated, purified and recovered for 1 hour. 10,000 cells were used for the assay. Crude nuclei preparation was achieved by incubating the cells in ATAC lysis buffer. The pelleted nuclei were incubated with transposition reaction buffer containing Tn5 transposases and incubated at $37{ }^{\circ} \mathrm{C}$ for $30 \mathrm{~min}$, followed by DNA purification using Qiagen MinElute PCR purification Kit. Further, transposed DNA was barcoded and amplified through PCR. After quality control by Agilent Bioanalyzer 2100 and size-selection, samples were prepared as $2 \mathrm{nmol} / \mathrm{L}$ and sequenced on a HiSeq 2500 (Illumina, AG Fischer, DZNE).

Paired-end $75 \mathrm{bp}$ reads were aligned to genome assembly MGSC $37 / \mathrm{mm} 9$ by Bowtie 2 and peaks were called by MACS 2 with FDR $<0.05$. Differential enrichment of peaks was analyzed using DiffBind package in R. Annotation of genomic regions with accessibility compared to whole genome was performed using Cis-regulatory Element Annotation System (CEAS). Distance to TSS plot was generated by GREAT using basal plus extension association rule: $1 \mathrm{~kb}$ upstream, $1 \mathrm{~kb}$ downstream, plus $1000 \mathrm{~kb}$ extension

\subsubsection{RNA-seq library preparation and data analysis}

For RNA-seq performed in murine primary acinar cells, acinar cells were isolated as described in the method part 2.2.2.5 and RNA were isolated as described in part 2.2.3.2. 500ng RNA was used for RNA-seq library preparation from two $p 48$-Cre mice (one $p 48$ Cre mouse was excluded from the analysis as the sample failed to reach the starting quantity of RNA-seq library preparation) and three Arid1a $a^{f / f f} ; p 48-C r e$ mice. For RNA-seq conducted in 266-6 cells, cells were seeded in 6-well plates at the density of 550,000 cells/well for three biological replicates per condition. Arid1a silencing and EGF treatment was conducted as described above. Total RNA was harvested by TRIzol method and quality was assessed by running 500 ng RNA on an agarose gel. Libraries were prepared from 500 ng RNA utilizing Truseq RNA Library Prep Kit v2 following manufacturer's instructions. mRNA was captured by RNA purification beads and eluted. The first strand and second strand cDNA were synthesized and the overhangs of the fragments were repaired into blunt ends. The 3' end was added with a single adenine to prevent mutual ligation during the following adapter conjugation. Finally, the DNA fragments with both flow-cell-hybridization adapters were amplified and the library was quantified by Agilent Bioanalyzer 2100. Size selection was optional if the main population 
of the library exceeds the appropriate size for sequencing or small fragments such as primer-dimers exist.

Approximate 20-30 million single-end $50 \mathrm{bp}$ reads/sample were sequenced on a HiSeq 2000 (for acinar cell RNA-seq) or a HiSeq 4000 (for 266-6 cell RNA-seq) (Illumina) at NIG, UMG. Analysis of RNA-seq was performed on Galaxy|Europe (usegalaxy.eu) platform (Afgan et al., 2018). The reads were mapped to mouse $\mathrm{mm} 9$ genome by TopHat (Version 2.1.1; Kim et al., 2013) and transcripts were counted by htseq-count (Version 0.9.1; Anders et al., 2014). Transcripts normalization and differential expression analysis were performed by Cuffnorm (Version 2.2.1.2) and Cuffdiff (version 2.2.1.5) with FDR cutoff 0.05 (Trapnell et al., 2010) and shown as fragments per kilobase million (FPKM). The quality of RNA-seq was assessed by PCA plot generated by plotPCA function in R utilizing htseq-count files.

Heatmaps were generated by pheatmap package in R using FPKM-converted z-score with K-means clustering. The acinar- and ductal- markers shown in customized heatmap were defined from single-cell transcriptome analysis by Muraro et al. and Hosein et al. (Muraro et al., 2016, Hosein et al., 2019). Gene Set Enrichment Analysis of acinar cell RNA-seq was performed using GSEA_4.0.3 software with C2_cgp (chemical and genetic perturbations) gene sets in MSigDB and literature exposited signatures (Boj et al., 2015; Jiang et al., 2016; Krah et al., 2015). For GSEA analysis of acinar RNA-seq and ATACseq overlay genes, genes that gained openness in Arid1a ${ }^{f / f t} ; p 48-C r e$ mice (fold change greater than 0 ) with FDR threshold 0.25 were selected from the RNA-seq expression profile and aligned with C2_cgp molecular signature database. Venn diagrams were drawn by Gene List Venn Diagram (genevenn.Sourceforge.net). Gene ontology from GREAT outputs was analyzed by Gene Ontology Consortium (http://geneontology.org/). Biological process enrichment analysis was carried out by default settings. Genes downregulated upon NFATC1-knockdown was selected with log2 fold change < 0 and FDR threshold 0.1 from RNA-seq analysis provided by M. Hasselluhn (Hasselluhn et al. 2019)

\subsubsection{Statistical analysis}

All the experiments were performed in technical triplicates using at least two independent biological replicates. Data were presented as mean \pm s.d. and GraphPad Prism 8 was employed for statistical analysis. For acinar explant in vitro culture with three-genotyped 
mice, the effects of different genotypes were calculated by two-way analysis of variance (ANOVA) followed by Tukey's multiple comparison test. Survival analysis in KaplanMeier curve was conducted by Log-rank (Mantel-Cox) test. For the rest of the analysis, statistical analysis was performed by two-tailed student's $t$-test. Statistical significance was considered as ${ }^{*} P<0.05,{ }^{* *} P<0.01,{ }^{* * *} P<0.001,{ }^{* * *} P<0.0001$. 


\section{Results}

\subsection{ARID1A is required to maintain pancreatic acinar cell identity and prohibits pre-neoplastic lesion formation}

\subsubsection{Arid1a deficiency causes acinar cell loss and duct dilation}

In order to dissect the role of ARID1A in maintaining the composition and function of the pancreas independent of oncogenic Kras, we generated a Cre-driven Arid1a-knockout mouse model under the regulation of the $p 48$ promoter which ensures and restricts the Arid1a depletion to acinar cells (Rose et al. 2001). The depletion of Arid1a in the acinar cell compartment was confirmed by immunohistochemistry (Figure 5A). The heterozygous Arid1 $\mathrm{a}^{\mathrm{fl} /+} ;$ p48-Cre mice were grossly normal (Figure 5B) and the pancreata were indistinguishable from the control cohorts until 1 year postnatal, when mucinous lesions were detectable (Figure 5C). In contrast, the homozygous littermates

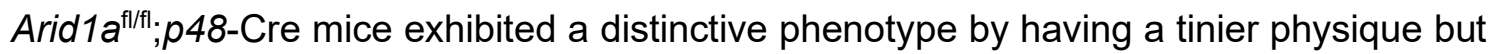
disproportionate swollen abdomen (Figure 5B). By having a significantly shortened median survival, a substantial portion (80\%) of the Arid1a $a^{f / f t} ; p 48$-Cre mice became moribund within 3 months postnatal while Arid $1 a^{f / /+} ; p 48-C r e$ and $p 48-C r e$ mice survived through 1 year (Figure 6A). Necropsy of the homozygous cohort revealed dilated gut with food impaction, implying towards exocrine pancreas insufficiency (Figure 6B). In line with the phenotype of functional pancreas impairment, weight loss of the pancreas was observed in Arid1 $a^{f / f t} ;$; $48-C r e$ populations, as reflected by the decreased pancreasto-body-weight ratio (Figure $6 \mathrm{C}$ ). 
A
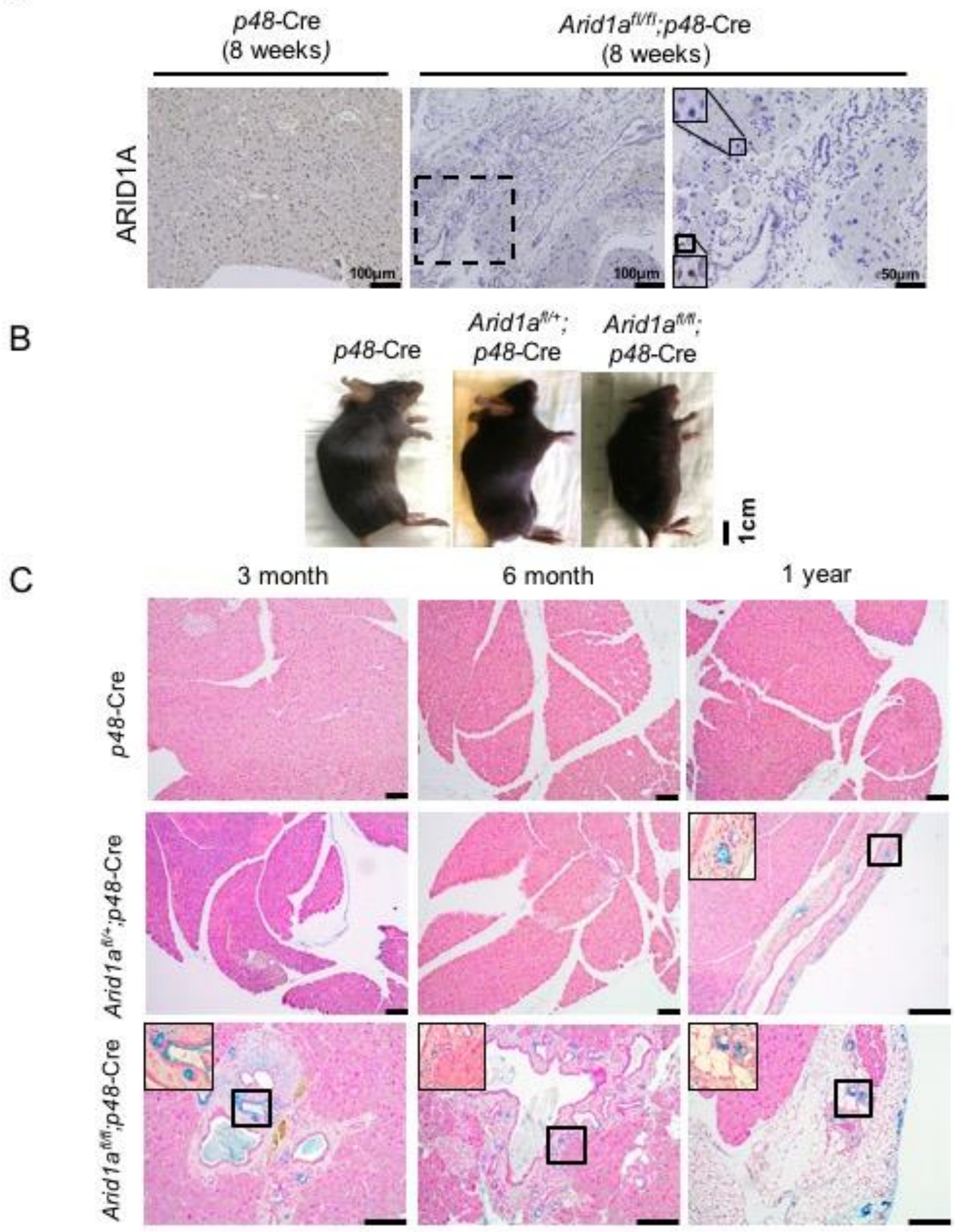

Figure 5. Arid1a deficiency affects mice pancreas in a dose-dependent manner. (A) ARID1A IHC of 8 weeks old $p 48$-Cre, Arid1 $a^{f / /+} ; p 48$-Cre and Arid1 $a^{f / / f /} ; p 48$-Cre mice. (B) Representative images of 8 weeks old $p 48$-Cre, Arid $1 a^{f / /+} ; p 48$-Cre and Arid $1 a^{f / f f /} ; p 48$-Cre mice.

(C) Alcian blue staining in the pancreas of mice of indicated genotype and age. 
A

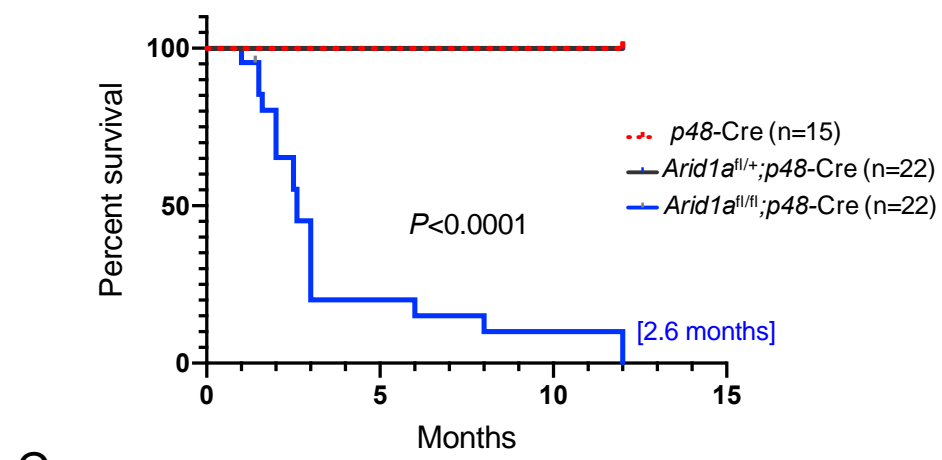

B

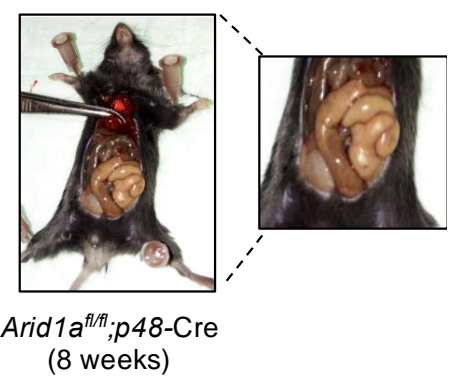

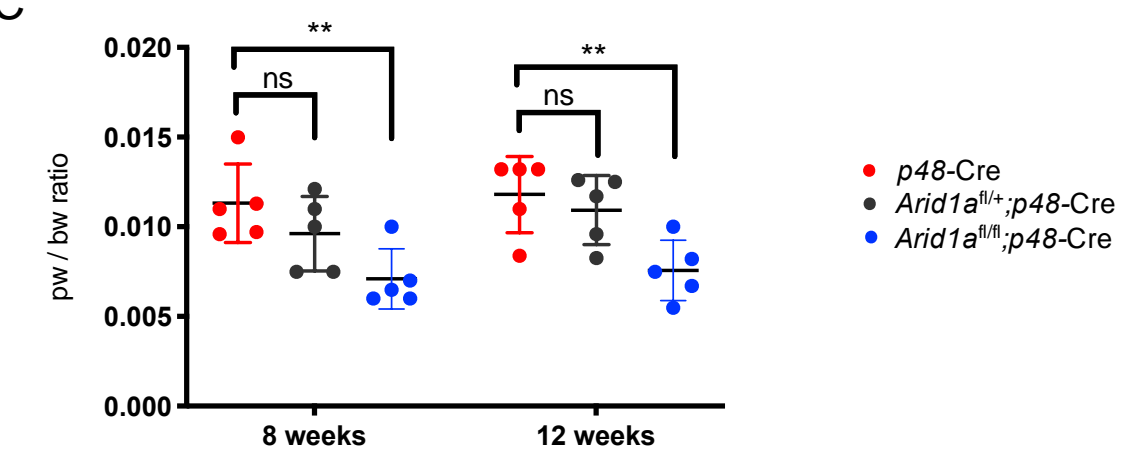

Figure 6. Arid1a deficiency leads to exocrine insufficiency phenotype of mice. (A) Kaplan-

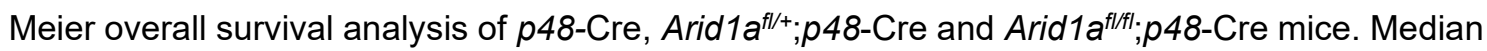
survival of the homozygous Arid1a-knockout cohorts is shown in brackets. (B) Representative image of swollen gut found in a Arid1a ${ }^{f / f f} ; p 48$-Cre survival mouse. (C) Scatter dot plot (mean \pm s.d.) showing the relative pancreas weight (pw) to body weight (bw) ratio of $p 48-C r e$, Arid $1 a^{f / /+} ; p 48-C r e$ and Arid $1 a^{f / f t} ; p 48-C r e$ mice. Statistical significance was determined by two-tailed unpaired Student's $t$-test. ns, not significant. $\mathrm{n}=5$ mice/genotype and time point. ${ }^{*} P<0.01$. Statistical significance was determined by Log-rank (Mantel-cox) test.

Next, we analyzed histologic changes of the pancreas by comparing H\&E and alcian blue staining between the Arid1a knockout- and wildtype- cohorts. Arid1a ablation caused macroscopic cystic vesicles in the pancreas, along with acinar-to-ductal metaplasia (ADM) and early stage PanIN lesions, of which the acid mucins are stained by alcian blue (Lee et al. 2017) (Figure 7A). Quantification of the range of ADM and PanIN area was performed based on morphology and alcian blue positivity (Figure 7B). 
A

p48-Cre

(8 weeks)
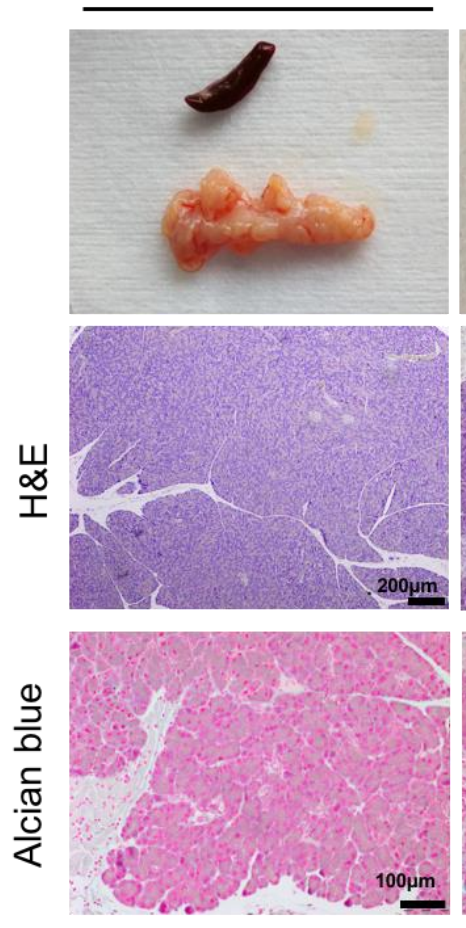

B

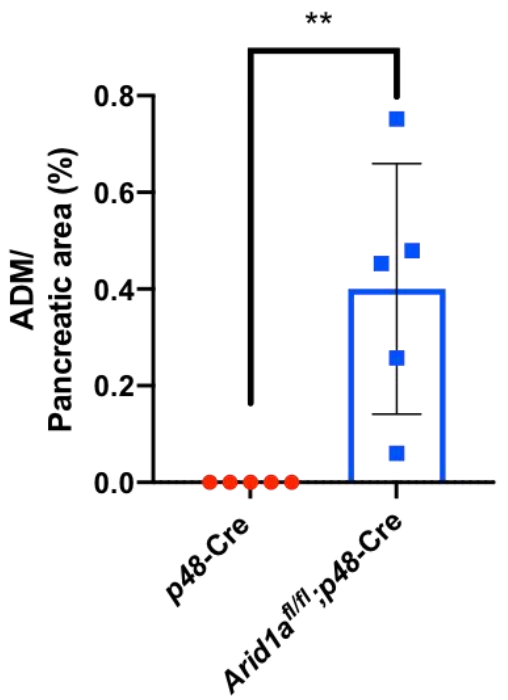

Arid1a ${ }^{f / f t} ;$ p48-Cre

(8 weeks)
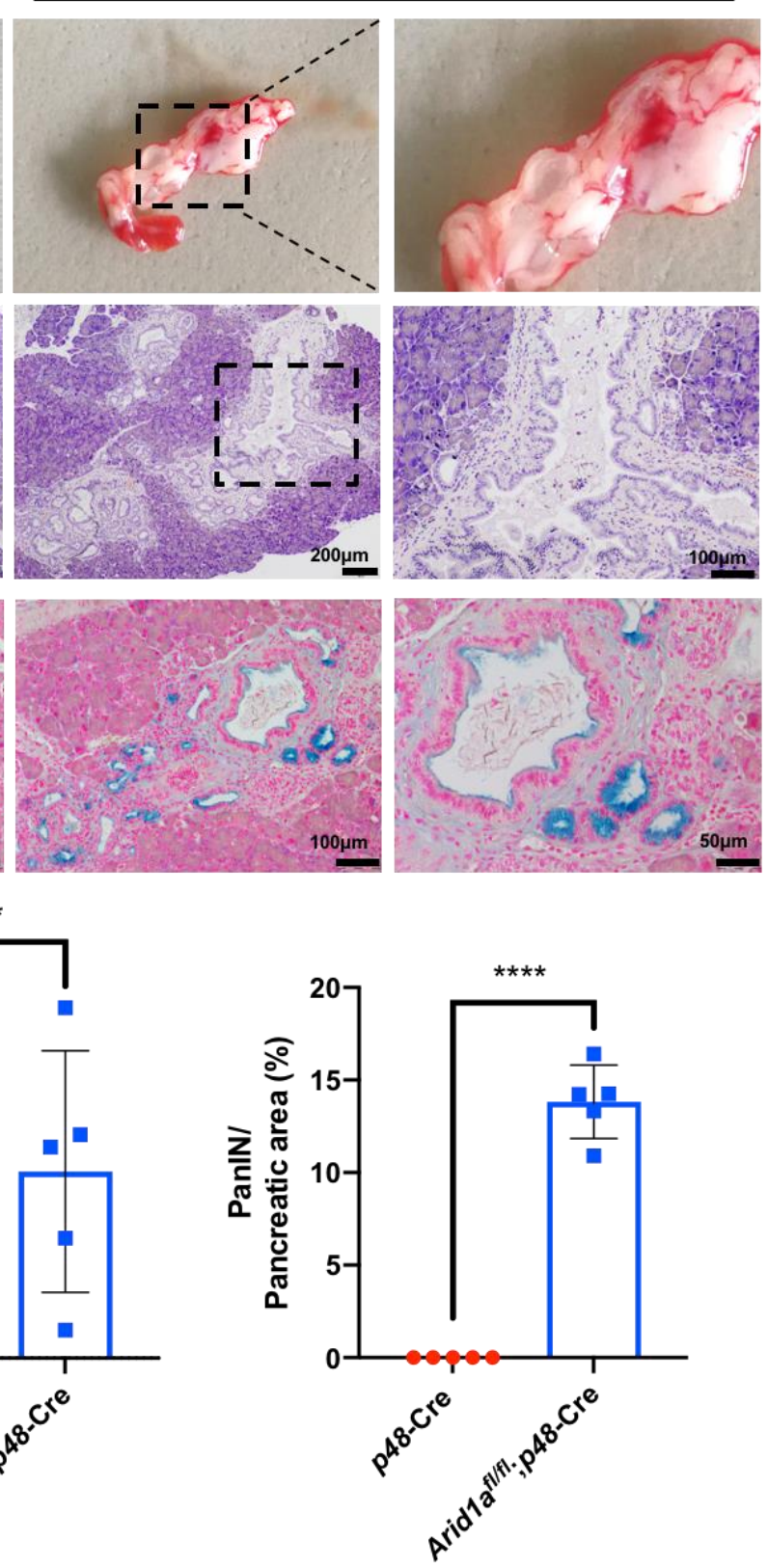

Figure 7. ARID1A is necessary for maintaining pancreatic acinar cell integrity. (A) Macroscopic images with corresponding H\&E- and immunohistochemical stainings in mice of indicated genotypes. (B) Scatter-dot plots showing the percentage of the pancreatic area containing ADM (left) and PanIN lesions (right) in 8 weeks old $p 48$-Cre and Arid $1 a^{f / f / t} ; p 48$-Cre mice. Data in $B$ are presented as mean \pm s.d. ( $n=5$ mice) and two-tailed unpaired $t$-test was used to determine significance. ${ }^{* \star} P<0.01,{ }^{* * \star *} P<0.0001$. 
Further characterization of the Arid $1 a^{f / / f /} ; p 48-C r e$ pancreas verified the ductal property of the metaplastic lesions with reduced staining of acinar markers AMYLASE and MIST1 and upregulated CK19, a ductal-specific marker (Figure 8). With age, Arid1 $a^{f / f f} ;$; $48-C r e$ mice continued to lose acinar cell mass and displayed wide-spread adipocyte infiltration, which implied as a feature of pancreatic atrophy (Figure 5C). Consistent with the literature demonstrating the correlation of inflammation to acinar reprogramming (Morris et al. 2010), we observed an enrichment of CD45-positive immune cells in the pancreatic stroma (Figure 8). Together, these findings suggest that Arid1a deficiency impairs the integrity of the acinar cell compartment and induces pre-neoplastic ADM and PanIN formation in the pancreas. However, we could not detect high-grade PanIN or PDAC in mice lacking Arid1a, suggesting that Arid1a deficiency alone is not sufficient for the progression to high-grade dysplasia or carcinoma.
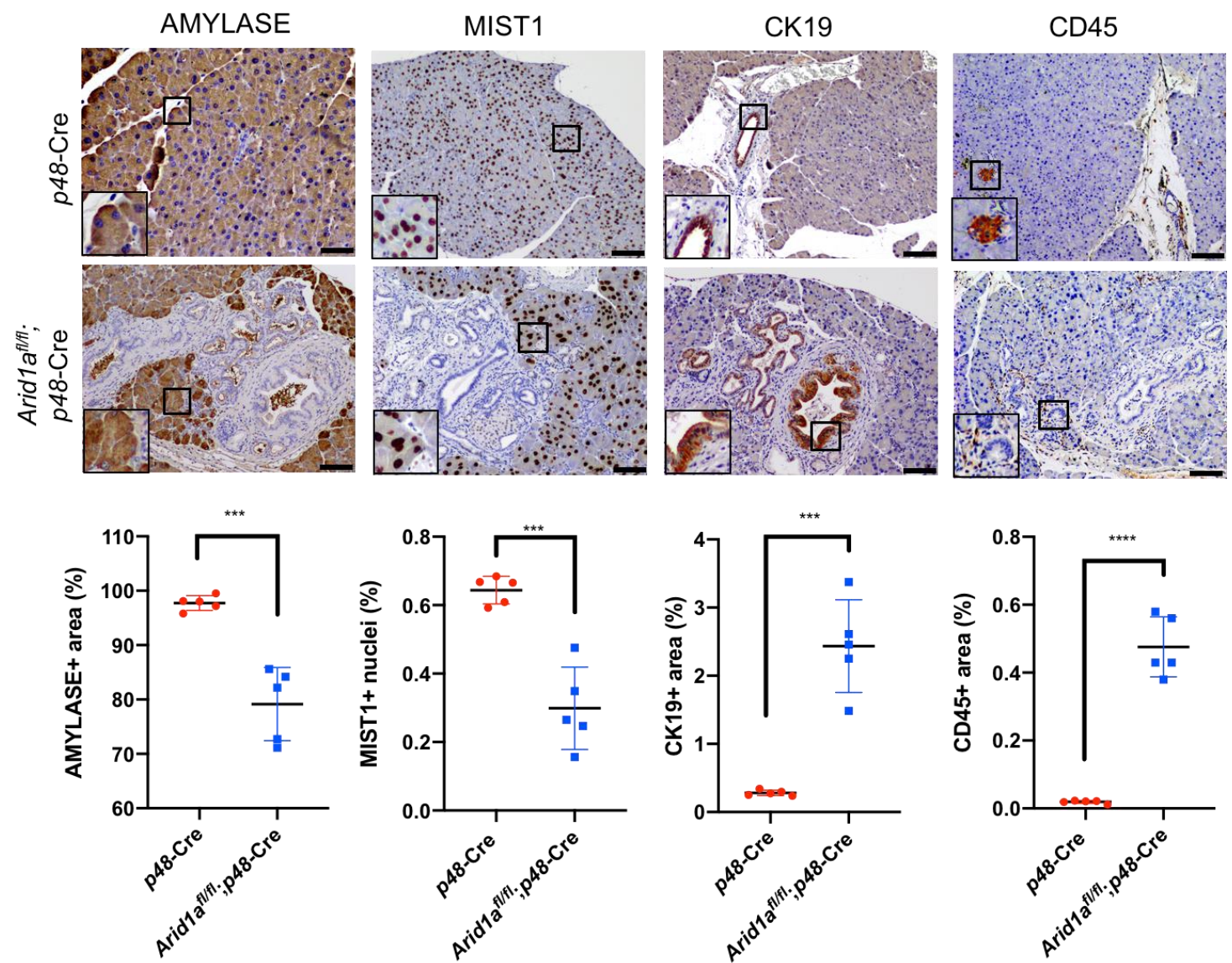

Figure 8. Arid1a loss induces ductal-lesion formation and inflammation infiltration. Representative images and corresponding quantification of indicated immunohistochemical stainings performed in 8 weeks old mice. Scale bars: $100 \mu \mathrm{m}$. Data are presented as mean \pm s.d. ( $n=5$ mice) and two-tailed unpaired $t$-test was used to determine the significance. ${ }^{* * *} P<0.001$, **** $P<0.0001$. 


\subsubsection{Arid1a loss caused transcriptional shifts in pancreatic acinar cell identity}

In order to understand the mechanism which leads to acinar reprogramming in Arid1 $a^{f / f / l} ; p 48-C r e$ mice, we performed genome-wide transcriptional analysis via RNAsequencing (RNA-seq) in primary acinar cells isolated from 8 weeks old p48-Cre and Arid1a ${ }^{f / f / l} ; p 48-C r e$ mice after confirming Arid1a depletion on mRNA level (Figure 9A, B). One $p 48$-Cre sample was excluded from the analysis as the amount of available material was insufficient for RNA-seq library preparation. Principle component analysis (PCA) showed two segregated clusters formed by replicates of either genotype (Figure 9C).

A

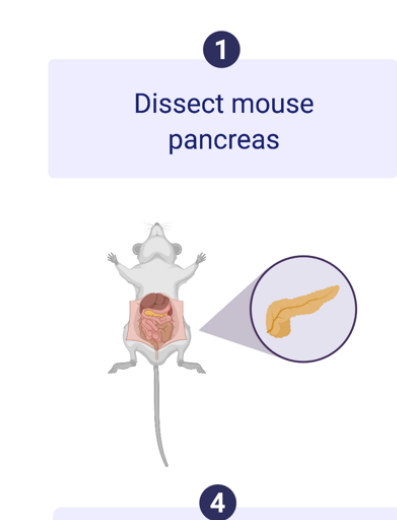

Digest with collagenase

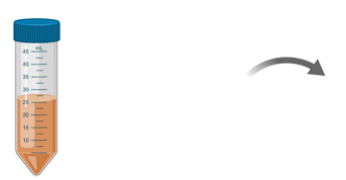

B

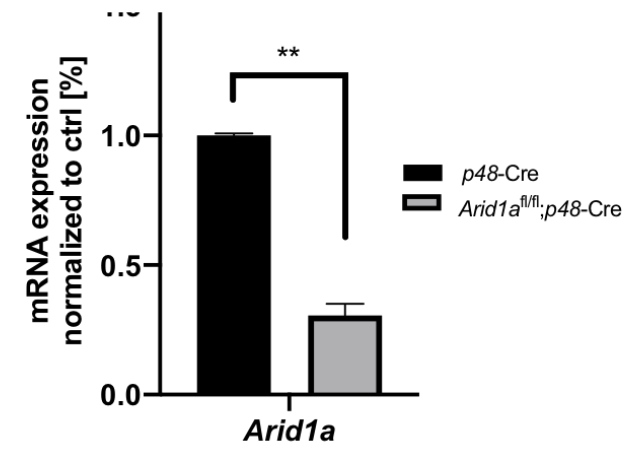

2

Physical crushing

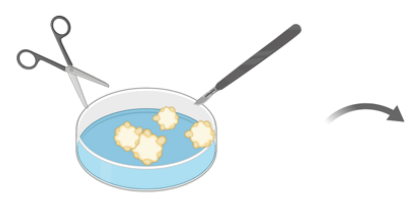

5

Fllter through $100 \mu \mathrm{m}$ strainer

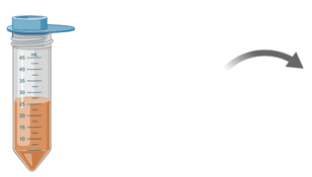

3

Centrifuge to remove blood vessels and fat

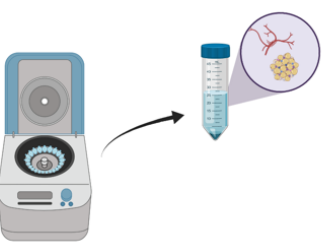

6

RNA-seq/ATAC-seq or culture for ADM formation

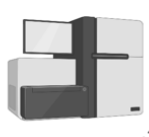

PCA_acinar cell RNA-seq

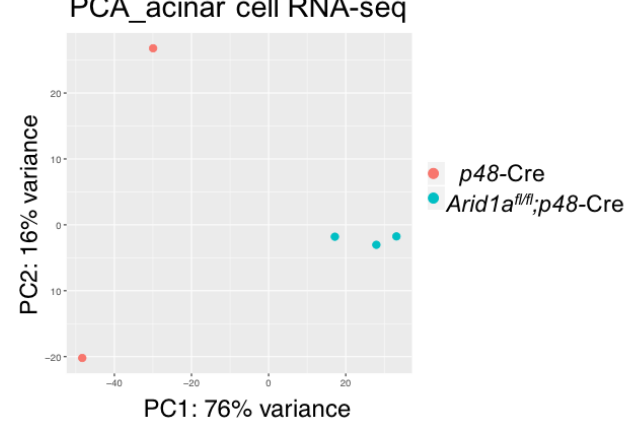

Figure 9. Conventional diagram of acinar cell isolation and quality assessment of RNAseq. For legend, see next page. 
Conventional diagram of acinar cell isolation and quality assessment of RNA-seq. (A) The schematic representation of the primary acinar cell isolation procedure from mice pancreas created with BioRender. (B) Acinar cells isolated from each 3 mice of the indicated genotypes were subjected to qRT-PCR-analysis to determine Arid1a expression prior to RNA-sequencing (normalized to $R p / p 0$ ). Statistical significance was analyzed by unpaired Student's t-test. ( $p 48-$ Cre $\mathrm{n}=2$, Arid1a ${ }^{\mathrm{flfl} f \mathrm{p}} ; p 48$-Cre $\mathrm{n}=3$ mice). ${ }^{*} P<0.01$. (C) PCA of acinar cell RNA-seq showing the separate clusters of the two genotypes.

Unsupervised hierarchical clustering of genes passing threshold (FDR $<0.05$ and FPKM $>5$ ) revealed distinct transcriptome alterations between the two genotypes. We saw that 2745 out of 3551 genes were up-regulated upon Arid1a deletion (Figure 10A). Consistent with the phenotype of Arid1a ${ }^{f / f t} ; p 48$-Cre mice, gene set enrichment analysis (GSEA) revealed an activation of pre-neoplastic-lesion- and inflammation programs, such as IL6 signaling and genes enriched in PanIN organoids, and a down-regulation of genes that maintain intact acinar cell homeostasis in Arid1a-knockout samples (Boj et al. 2015) (Figure 10B). Further, and consistent with its involvement in ADM (Russell et al. 2015) and PanIN-formation (Wang et al. 2017), gene sets associated with epithelial-tomesenchymal transition (EMT) were also enriched in Arid1a $a^{f / f t} ; p 48-C r e$ acinar cells. Besides, GSEA also detected genes that are upregulated in mice with depletion of Ptf1a, an acinar identity maintaining gene, to be enriched in mouse lacking Arid1a, suggesting a comparable function of ARID1A in modulating acinar transcriptional network. Thus, ARID1A hinders transcriptional pathways that direct acinar cell dedifferentiation and is essential for maintaining acinar homeostasis of the pancreas. 
A

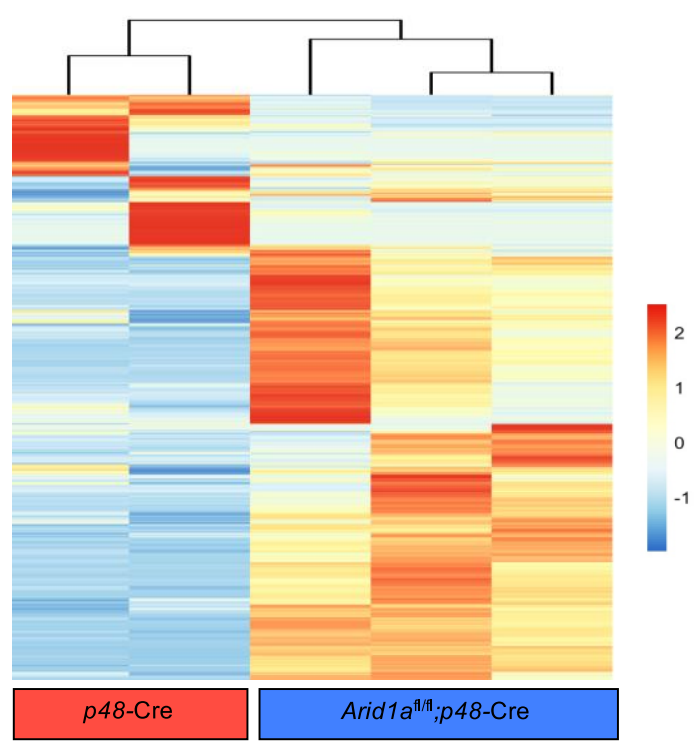

B

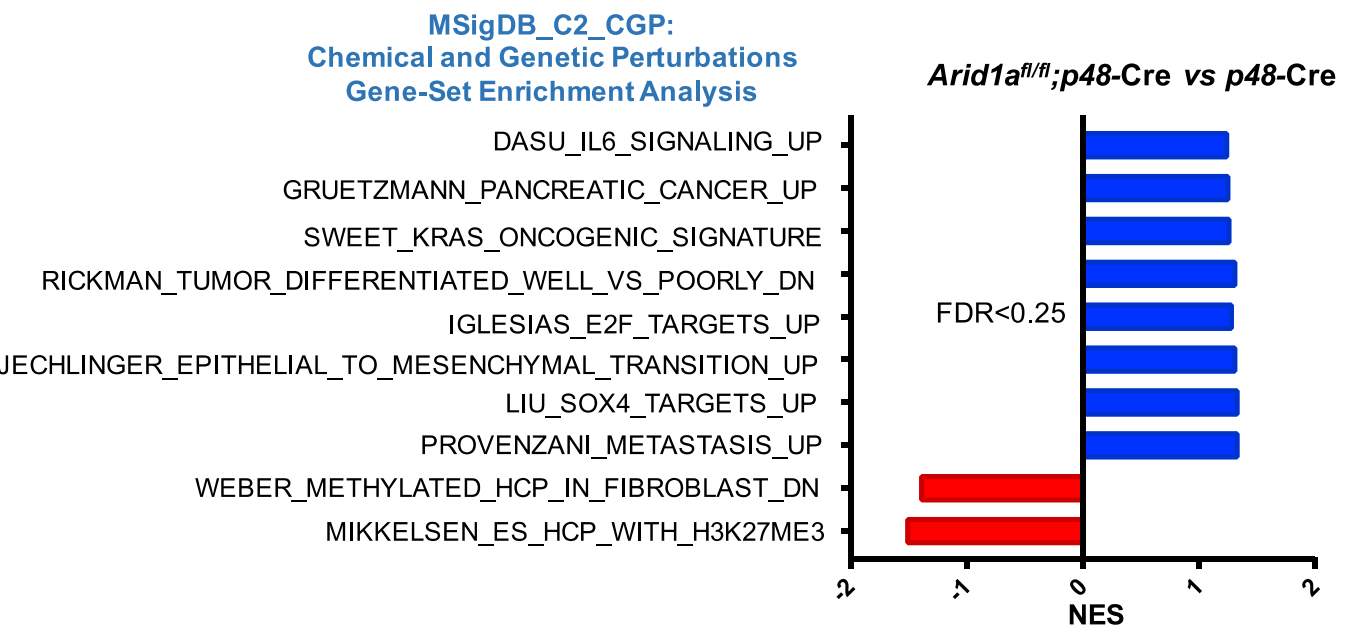

Figure 10. Arid1a loss activates signaling pathways promoting acinar cell dedifferentiation. (A) Heatmap displaying FPKM-transformed $(F P K M>5) z$ scores upon RNA-seq analysis in acinar cells of 8 weeks old $p 48$-Cre- and Arid $1 a^{f / f / l} ; p 48$-Cre mice. (B) Top differentially enriched gene sets in MSigDB database (C2-Chemical and genetic perturbations, top) and alignment of the literatureexposited datasets (bottom) with RNA-seq data by Gene Set Enrichment Analysis (GSEA). All the considered alignments are with False Discovery Rate (FDR) $<0.25$. Normalized Enrichment Score (NES) and FDR are indicated on the graph. 


\subsection{Arid1a loss increases chromatin accessibility}

Given the transcriptional shift induced by Arid1a loss as revealed by RNA-seq, we hypothesized that Arid1a deficiency alters physical compaction of chromatin thus affecting gene expression. To detect ARID1A-depedent genome-wide chromatin accessibility, we performed assay for transposase-accessible chromatin followed by high-throughput sequencing (ATAC-seq) (Buenrostro et al., 2013) utilizing acinar cells isolated from the same donors as RNA-seq. PCA was performed for quality assessment and showed two separate chromatin profile clusters formed by either genotype (Figure 11A). As shown in Figure 11B, Arid1a loss led to a more accessible chromatin. The genomic regions that gained openness upon Arid1a knockout were mainly 5' UTR and promoter regions within $1 \mathrm{~kb}$ adjacent to a transcription start site (TSS) (Figure 11C, D).

A

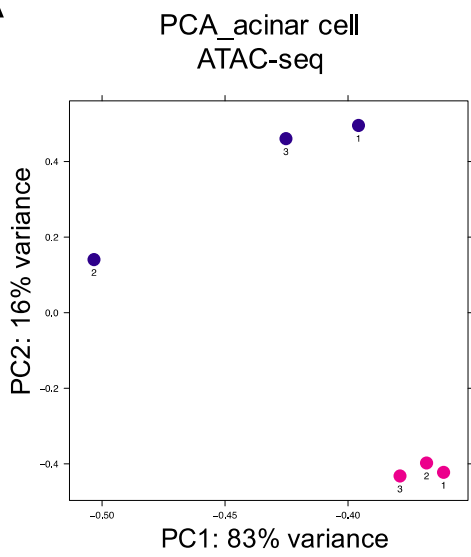

C

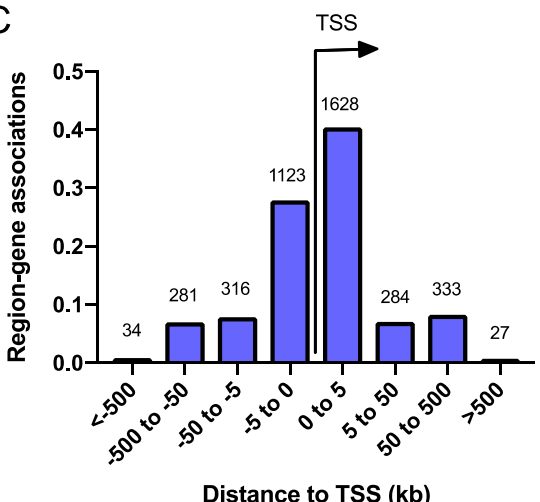

B

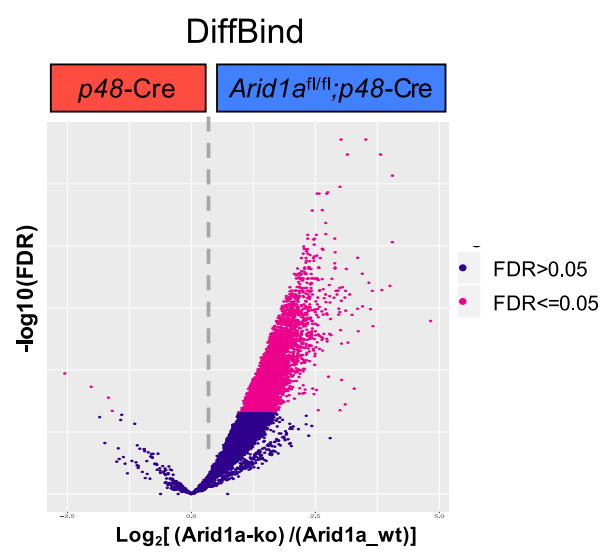

$\mathrm{D}$

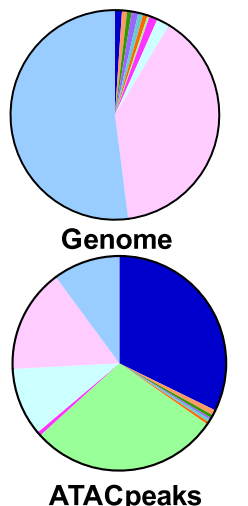

Promoter(<=1000 bp) $\square$ Promoter(1000-2000 bp)

Promoter(2000-3000 bp) $\square$ Downstream (<=1000 bp) $\square$ Downstream $(1000-2000 \mathrm{bp})$ $\square$ Downstream (2000-3000 bp) $\square$ 5'UTR $\square$ 3'UTR $\square$ Coding exon $\square$ Intron $\square$ Distal intergenic

Figure 11. Arid1a deficiency increases chromatin accessibility. (A) PCA analysis of ATACseq. (B) Volcano plot of Differential Binding Analysis of ATAC-seq peak profile. (C) Bar graph from GREAT depicting the distribution of ATAC-seq peaks as defined by distance to TSS. The analysis in GREAT was performed with basal plus extension association rule with default settings. (D) Genomic distribution of the annotated peaks obtained by ATAC-seq using Cis-Regulatory Annotation System (CEAS). 


\subsection{Arid1a deficiency cooperates with EGFR signaling in driving acinar cell reprogramming}

\subsubsection{Arid1a loss enhances EGF-induced acinar to ductal transdifferentiation}

To dissect the mechanism of acinar reprogramming controlled by Arid1a deficiency, we focused on genes that gain openness (fold change $>0, F D R<=0.25$ in ATAC-seq) in the Arid1a knockout condition. Pathway analysis of these genes by GSEA showed an increased activity of epidermal growth factor receptor (EGFR) signaling (Figure 12A). Previous studies have shown that activation of EGFR by its ligands such as EGF and TGFa promotes in vitro acinar-to-ductal conversion, while pharmacological interference with EGFR signaling sufficiently prevents this transdifferentiation process (Tobita et al. 2003; Ardito et al. 2012; Siveke et al. 2012; Chen et al., 2015). In line with the GSEA result, EGFR was observed to be upregulated in PanIN epithelial cells in Arid1a deficient pancreas (Figure 12B), suggesting a pattern of cooperation between Arid1a deficiency and EGFR signaling pathway in facilitating metaplastic lesions.

A

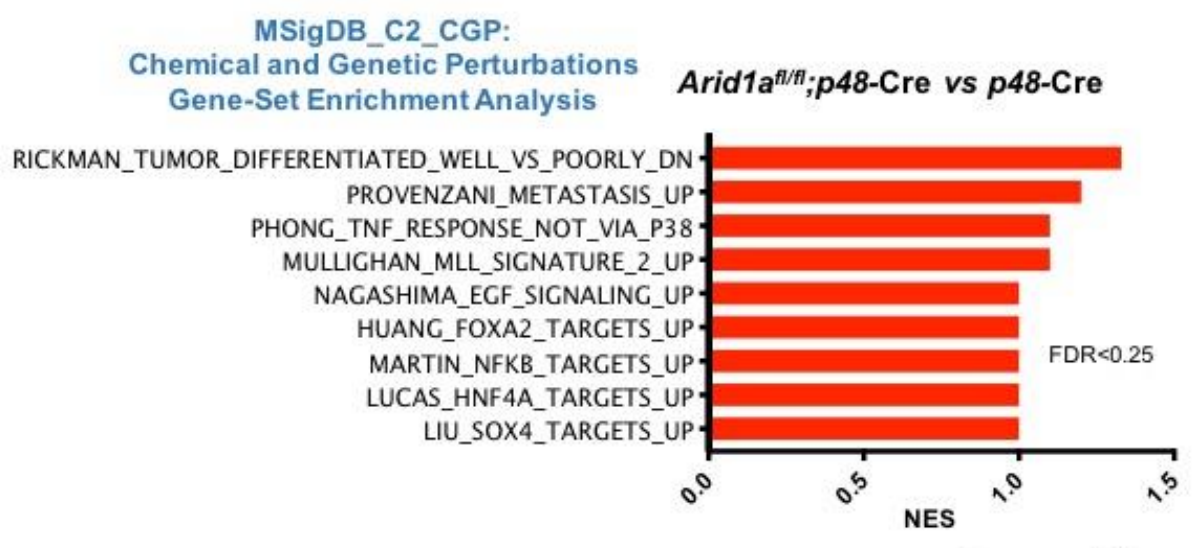

B

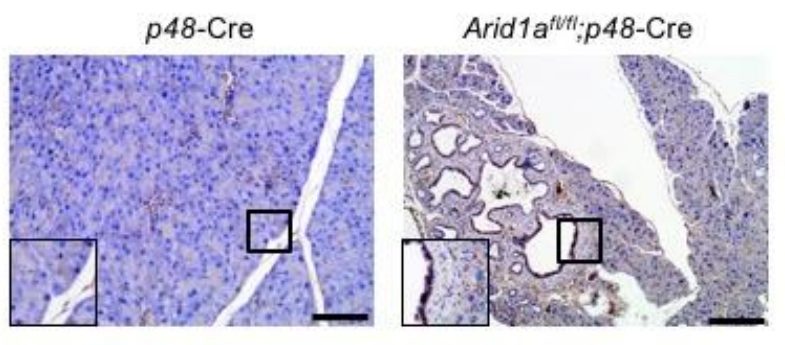

8 weeks

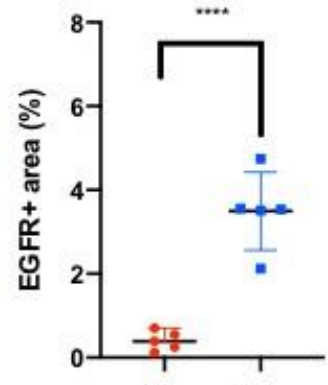

Figure 12. EGFR is overexpressed in Arid1a-deficient mice. For legend, see next page. 
EGFR is overexpressed in Arid1a-deficient mice. (A) GSEA performed for genes that gain openness in Arid1a-knockout mice as identified by ATAC-seq and show increased expression in Arid1 $a^{f / f / t} ; p 48$-Cre- vs $p 48$-Cre mice (fold change $>0$, FDR $<0.25$ ) using terms of chemical and genetic perturbations in MSigDB curated gene sets. (B) Representative images (left) and quantification (right) of EGFR IHC in 8 weeks-old $p 48$-Cre and Arid1 $a^{\text {fl/t/l}} ; p 48$-Cre mice ( $\mathrm{n}=5$ mice/genotype). Scale bars: $100 \mu \mathrm{m}$. Data in $\mathrm{B}$ are presented as mean \pm s.d. $(\mathrm{n}=5)$ with $P$-values shown on the top of each plot. ${ }^{* * *} P<0.0001$.

To provide additional evidence for the interaction of EGFR signaling and Arid1a deficiency, primary acinar cells isolated from p48-Cre, Arid $1 a^{\mathrm{f} / /+} ; p 48-C r e$ and Arid1 $a^{f / f f l} ; p 48-C r e$ mice were cultured in collagen-coated plates and allowed to form ADMs in the presence or absence of EGF. Arid1a loss actuated acinar cell conversion in a dose-dependent manner - cells with biallelic Arid1a depletion held higher capacity forming ADM, while ductal structures were rarely found in Arid1a wildtype cells (Figure 13A). In line with previous reports (De et al. 1990; Chen et al. 2015), EGF treatment induced ADM formation, notably however, Arid1a deficient cells showed a striking increase in the number of ductal-structures when exposed to EGF (Figure 13A). Together with the decreased Arid1a mRNA in Arid1a-deficient cells, qRT-PCR analysis detected increased expression of the ductal marker Krt18 and a downregulation of the acinar marker Amyl1 upon EGF treatment or/and Arid1a loss (Figure 13B). 
A

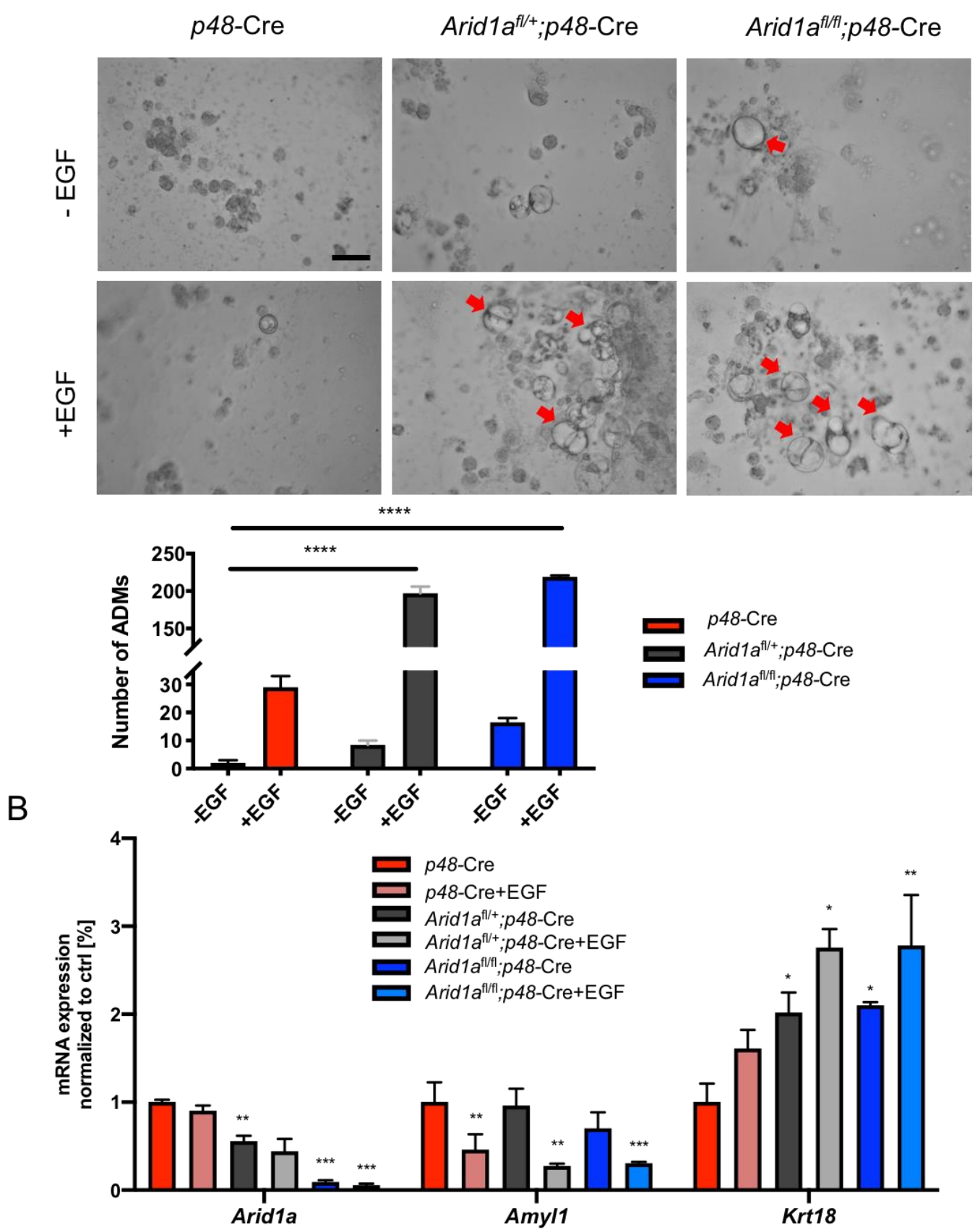

Figure 13. Arid1a deficiency cooperates with EGFR signaling in driving primary acinar cell reprogramming. (A) Acinar explants derived from $p 48-C r e$, Arid1 $a^{\mathrm{fl} / \mathrm{+}} ; p 48-\mathrm{Cre}$ and Arid1 $\mathrm{a}^{\mathrm{fll} / \mathrm{l}} ; p 48-$ Cre mice were cultured in vitro in the absence or presence of EGF $(40 \mathrm{ng} / \mathrm{ml})$ and were analyzed for ADM formation 5 days after seeding. The red arrows denote ADM structures. Scale bars: $50 \mu \mathrm{m}$. Quantification of the ADM numbers/well was performed in triplicate wells. (B) qRT-PCR analysis of Arid1a, Amyl1 and Krt18 (normalized to Rplp0) mRNA expression in ADM isolated at the fifth day after seeding and treatment. In A, statistical significance was determined by two-way ANOVA, followed by Tukey's multiple comparisons test between groups test. In B, two-tailed unpaired Student's $t$-test was used to calculate statistical significance between each data sample and the corresponding control. Data in A and B are presented as mean \pm s.d. $(n=2)$ with $P$-values shown on the top of each plot. ${ }^{*} P<0.05,{ }^{* *} P<0.01,{ }^{* * *} P<0.001,{ }^{* * * *} P<0.0001$. 
We utilized the murine Kras-mutant acinar cell line 266-6 as an additional model to verify our finding. Herein we observed that Arid1a silencing and induction of EGFR signaling are equally efficient in promoting acinar cell transdifferentiation. Comparable with the primary acinar cells, the highest number of ADMs was detected in the group with combined EGF treatment and Arid1a knockdown (Figure 14A). qRT-PCR confirmed the efficient knockdown of Arid1a in 266-6 cells (Figure 14B).

A

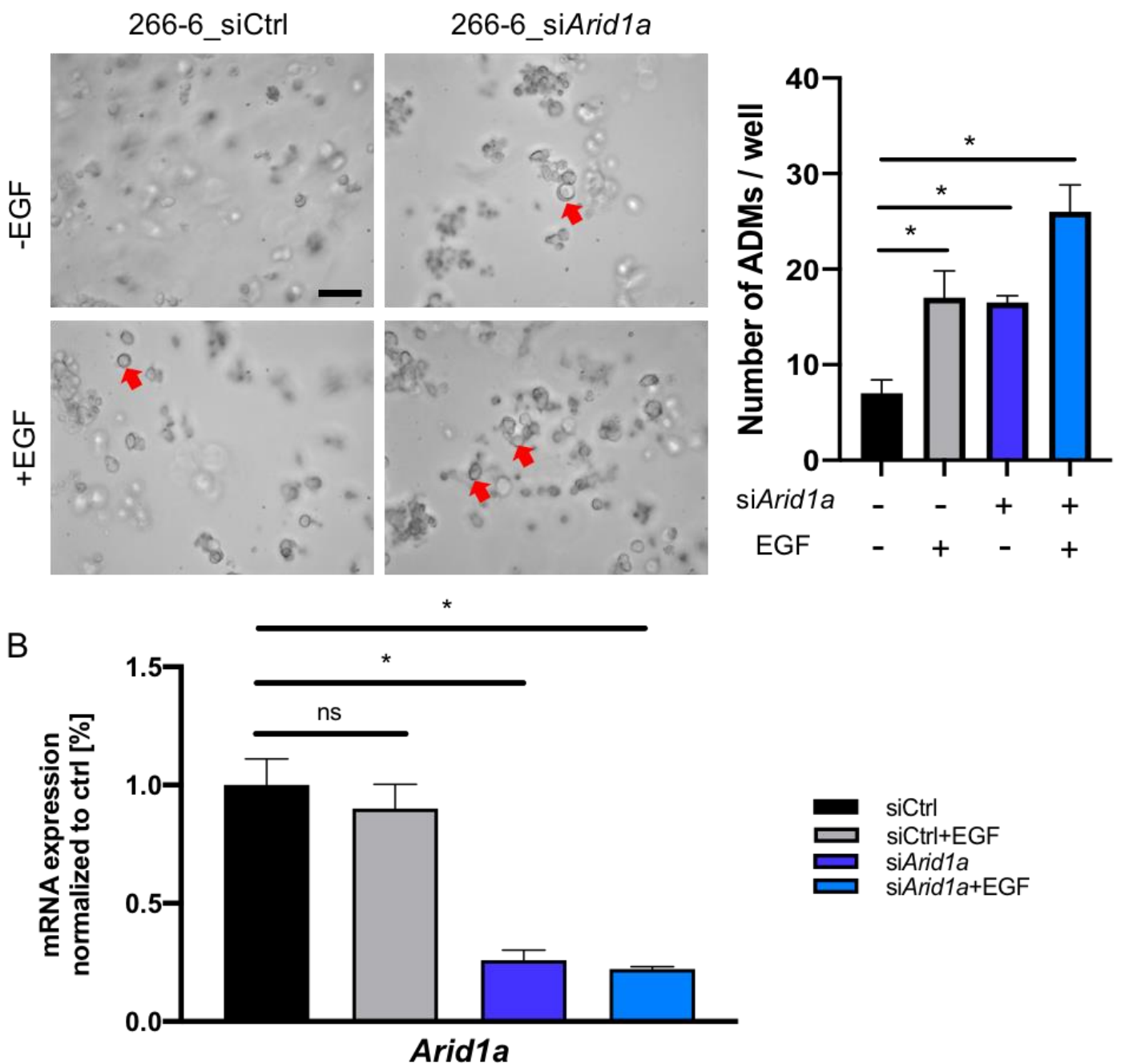

Figure 14. Arid1a deficiency cooperates with EGFR signaling in driving ADM in the context of oncogenic Kras. (A) Left: representative ADM culture of 266-6 cells in the absence and presence of Arid1a-knockdown and EGF stimulation. Red arrows point to the ductal-like structures. Scale bars: $50 \mu \mathrm{m}$. Right: Bar graph showing the quantification of ductal-structure numbers/well. (B) mRNA expression of Arid1a to confirm successful Arid1a knockdown in metaplastic structures four days after inducing ADM formation in 266-6 cells. In A and B, twotailed unpaired Student's $t$-test was used to calculate statistical significance between each data sample and the corresponding control. Data are presented as mean \pm s.d. $(n=2)$ with $P$-values shown on the top of each plot. ${ }^{*} P<0.05$. 
In order to capture the instant transcriptional alterations led by Arid1a silencing and EGFR signaling activation in ADM induction, RNA-seq was performed in Arid1a-intactand knockdown- 266-6 cells in the absence or presence of EGF. The sequencing quality was verified by four distinct clusters of each corresponding condition by PCA and the knockdown of Arid1a was confirmed by qRT-PCR (Figure 15A, B). We particularly focused on literature-curated acinar- and ductal-markers (FPKM $>0)$, which were differentially expressed in acinar- or ductal-compartments compared to other pancreatic cell types (Muraro et al.). Hierarchical clustering exhibited distinct expression patterns for ductal genes and acinar genes. Acinar genes were down-regulated by sole EGF treatment or Arid1a-knockdown and the expression of ductal genes was increased, while a vast switch from acinar to the ductal program was observed in the condition of concomitant Arid1a-knockdown and EGFR activation (Figure 15C). Notably, the cooperation of EGFR signaling and Arid1a loss was not applied to acinar gene repression, suggesting that the transcriptional induction of the ductal program is necessary for the prominent ADM phenotype observed upon combined Arid1a knockdown and EGFR activation.

A

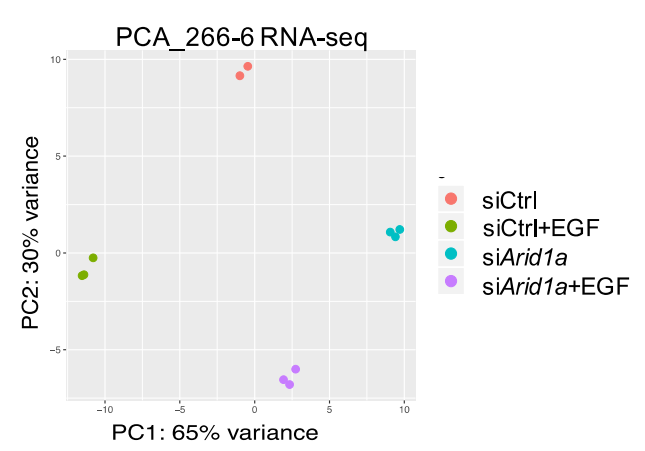

C

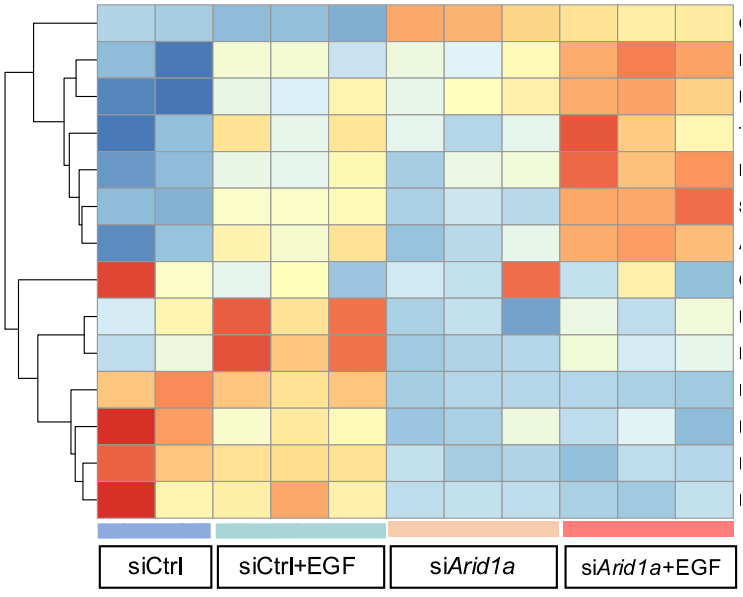

B
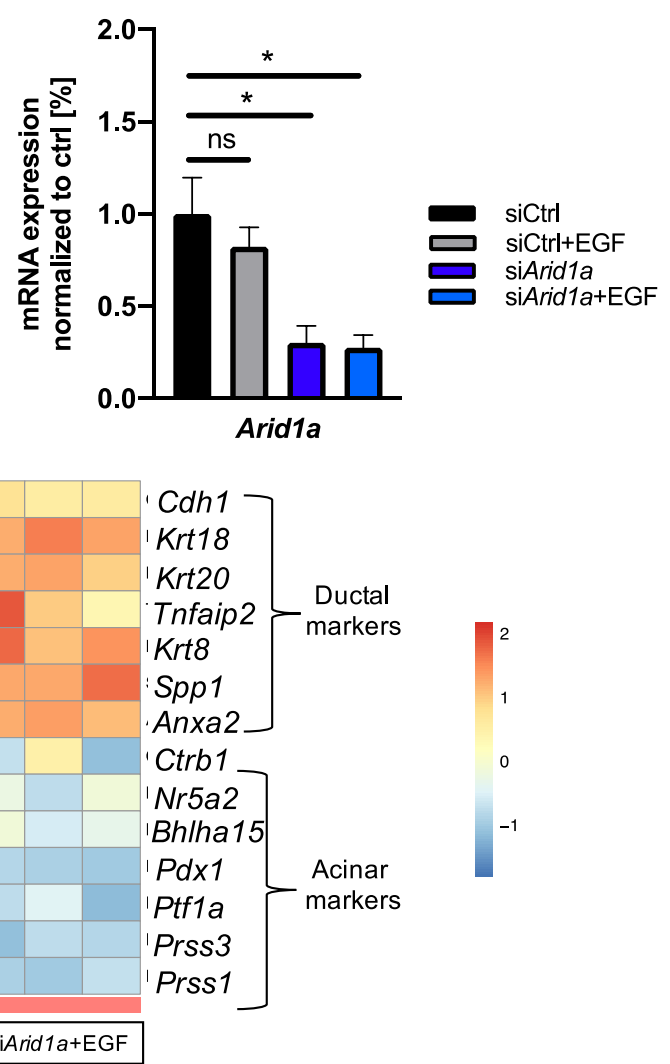

Figure 15. Alterations led by Arid1a-knockdown and EGFR activity in acinar and ductal markers expression. For legend, see next page. 
Alterations led by Arid1a-knockdown and EGFR activity in acinar and ductal markers expression. (A) PCA of acinar cell RNA-seq showing the separate clusters of the two genotypes. (B) Arid1a knockdown was validated in 266-6 RNA-seq samples as measured by qRT-PCR (normalized to Rp/p0). (C) Heatmap displaying z-scores of literature-curated (Muraro et al.) acinar and ductal markers determined by RNA-seq conducted in 266-6 cells in the presence and absence of Arid1a and EGF (FPKM>0, FDR<0.05).

\subsubsection{Detection of gene signatures driving cellular plasticity upon EGFR signaling activation and Arid1a deficiency}

We have shown that EGF stimulation and Arid1a deficiency cooperate in giving rise to $A D M$ in vitro. To further investigate the mechanistic insights of the interplay between EGFR activity and ARID1A, we sought to primarily identify the regulation pattern of each by clustering the whole-genome transcriptome profile with k-means method. It showed two subsets of genes which displayed cooperative upregulation or downregulation upon concomitant Arid1a knockdown and EGF stimulation (Figure 16A). Given that the cooperative effect of Arid1a deficiency and EGFR signaling activity were seen in boosting ductal-marker expression (Figure 15C), we focused on the cooperatively upregulated genes as they might potentially regulate the ductal program. By overlapping genes that gain expression upon EGF stimulation ( $\log 2 \mathrm{FC}>0$, FDR<0.05), Arid1a-knockdown and the combination of the two conditions, we got to know a list of 213 genes that fell in this category (Figure 16B). Gene Ontology analysis associated those 213 genes with biological processes such as animal organ regeneration and epithelial cell differentiation (Figure 16C), further verifying the involvement of these genes in driving cell plasticity. 
A

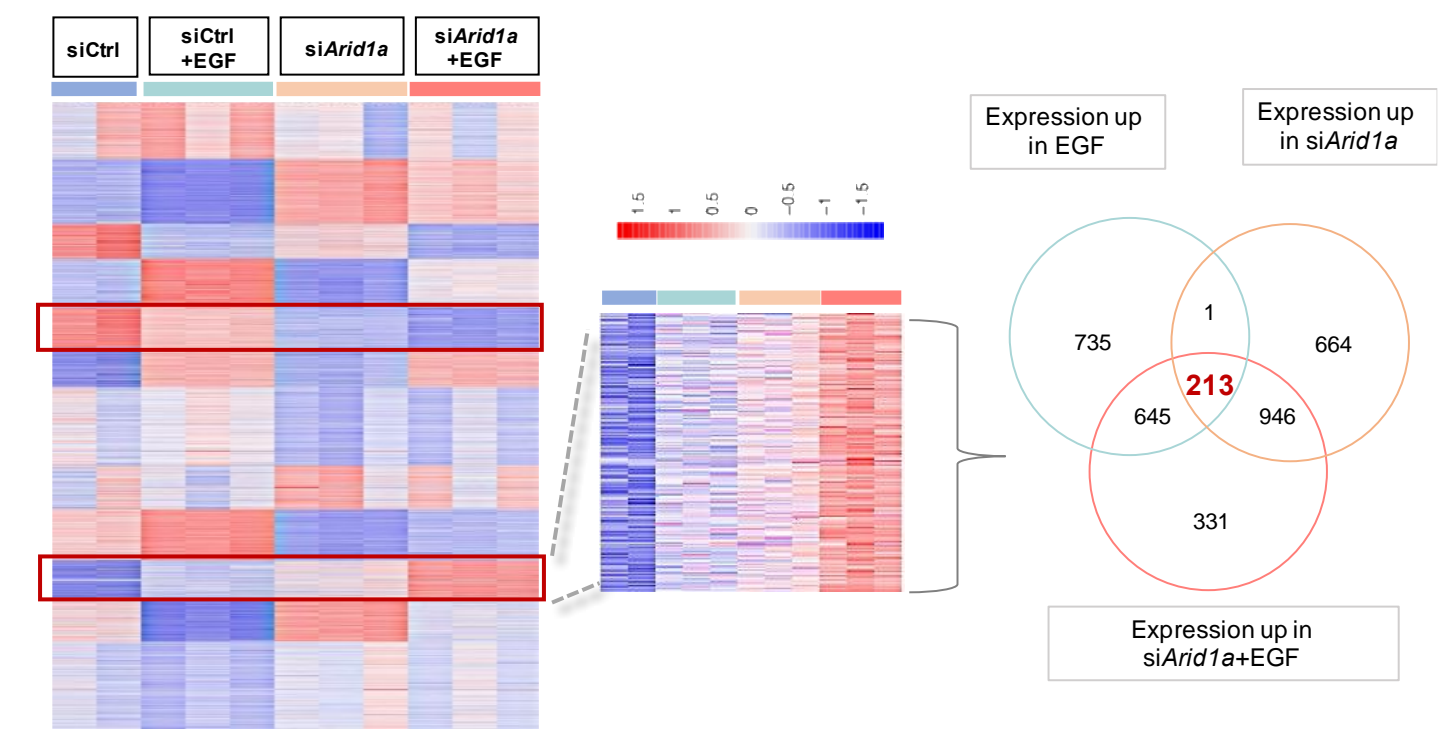

B

GO: Biological Process

Fold Enrichment
C

266-6 RNA-seq

213 commonly up-regulated genes

Positive chemotaxis

Animal organ regeneration

Extracellular matrix assembly

Regulation of collagen biosynthetic process

Epithelial tube morphogenesis

Regulation of epithelial cell differentiation

Positive regulation of cell morphogenesis

Cellular response to growth factor

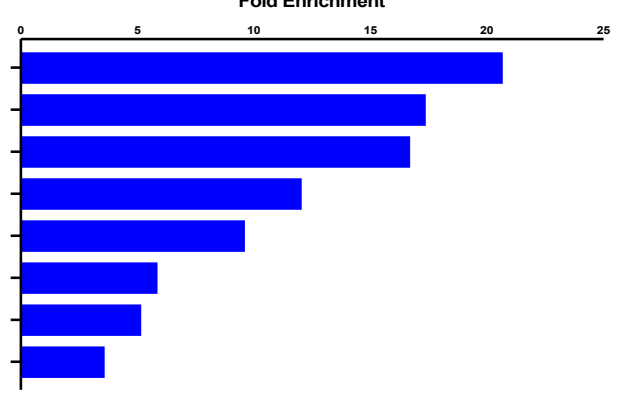

Figure 16. Arid1a deficiency and EGFR signaling cooperate for transcriptional activation of gene signatures driving cell plasticity. (A) Heatmap showing the k-means clustering $(k=12)$ of FPKM-transformed $z$-scores of the genes $(F P K M>0)$ in the indicated conditions upon RNA-seq studies in 266-6 cells. The magnification at the right side shows the clusters where EGFR activation and Arid1a-knockdown cooperate in regulating gene expression. (B) Venn diagram showing the overlap of up-regulated genes with either EGFR stimulation, Arid1a-knockdown or combined conditions compared to the control. Genes are selected as fold change $>0, F D R<0.05$. (C) Gene Ontology analysis exhibiting the top-ranked biological-process pathways of the 213 commonly-upregulated genes shown in B. Binomial test was performed and all the pathways are with FDR $p$ value $<0.05$. 


\subsubsection{ARID1A binds transcriptionally active promoters and enhancers}

Having observed a role of ARID1A in restricting ductal genes expression, we next asked whether this is, at least partially, due to the direct control of ARID1A. To capture the genome-wide localization of ARID1A, we conducted chromatin immunoprecipitation followed by sequencing (ChIP-seq) in 266-6 cells. The two replicate samples showed high consistency and finally 4253 peaks were called and related to 4253 genes. Alongside, we profiled $\mathrm{H} 3 \mathrm{~K} 27 \mathrm{ac}$, which indicates transcription activation both at proximal and distal regions of TSS (Shukla et al. 2008), in the condition of Arid1a-knockdown with or without EGFR activation. The chromatin profiles of the four conditions formed separated clusters as revealed by PCA (Figure 17A). ARID1A and H3K27ac peaks were highly correlated genome-widely, by having a 0.8 pearson correlation score (Figure 17B, C). Arid1a knockdown and sole EGF treatment caused genome-wide augmentation of the existing H3K27ac signal, particularly upon combining both conditions (Figure 17D). Consistently, global expression of H3K27ac was increased by EGF treatment and Arid1a-knockdown as well (data not shown). ARID1A peak was assigned to promoter when it was located within $1 \mathrm{~kb}$ vicinity of an annotated TSS or to enhancer when it colocalized with distal (>1kb +/-TSS) H3K27ac peaks, respectively. ChIP-seq revealed that ARID1A was bound to active chromatin enriched for H3K27ac and preferentially at H3K27ac-bound distal (68\% of the peaks) (Figure 18A, B). Given its priority of localizing at distal regions, enhancer-associated ARID1A peaks suggested a widespread role of ARID1A in regulating pancreatic identity (Figure 18C). Among the aforementioned 213 genes which were cooperatively up-regulated by Arid1a knockdown and EGF signaling activation, 81 genes (38\%) were identified to be direct ARID1A target genes (Figure 18D). Together, ARID1A co-localizes with transcriptional active elements and Arid1a deficiency causes hyper-acetylation of previously acetylated genes. 
A

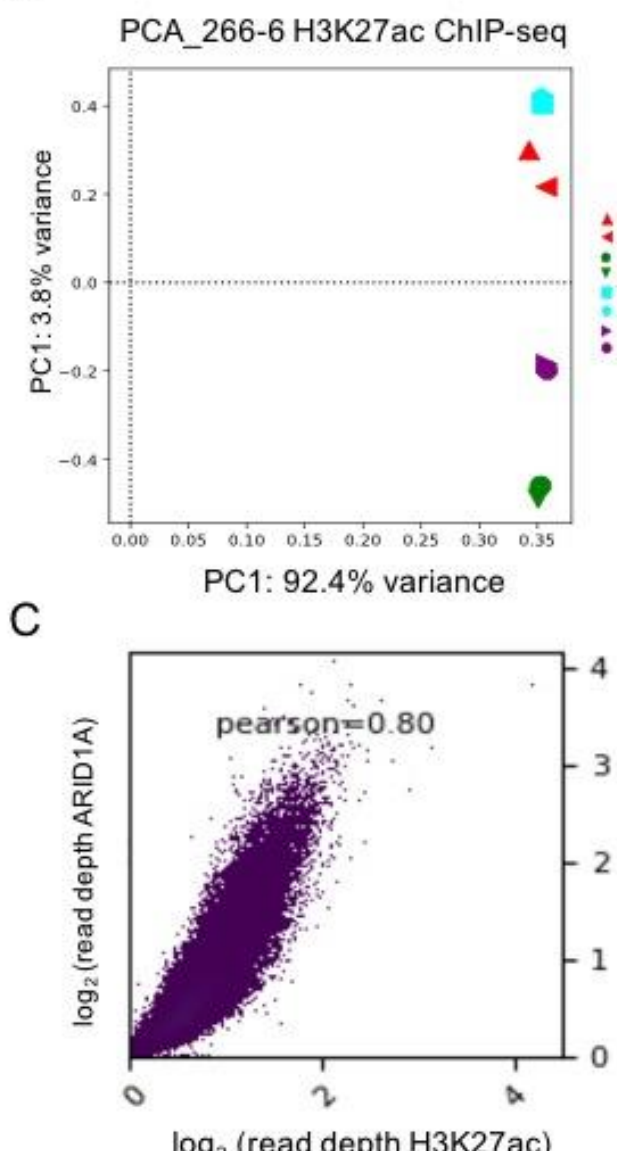

D

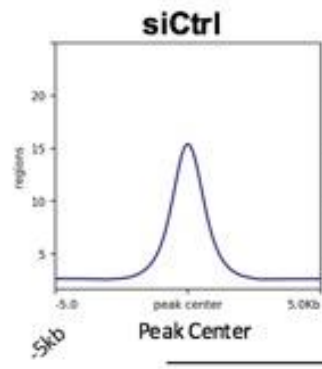

B

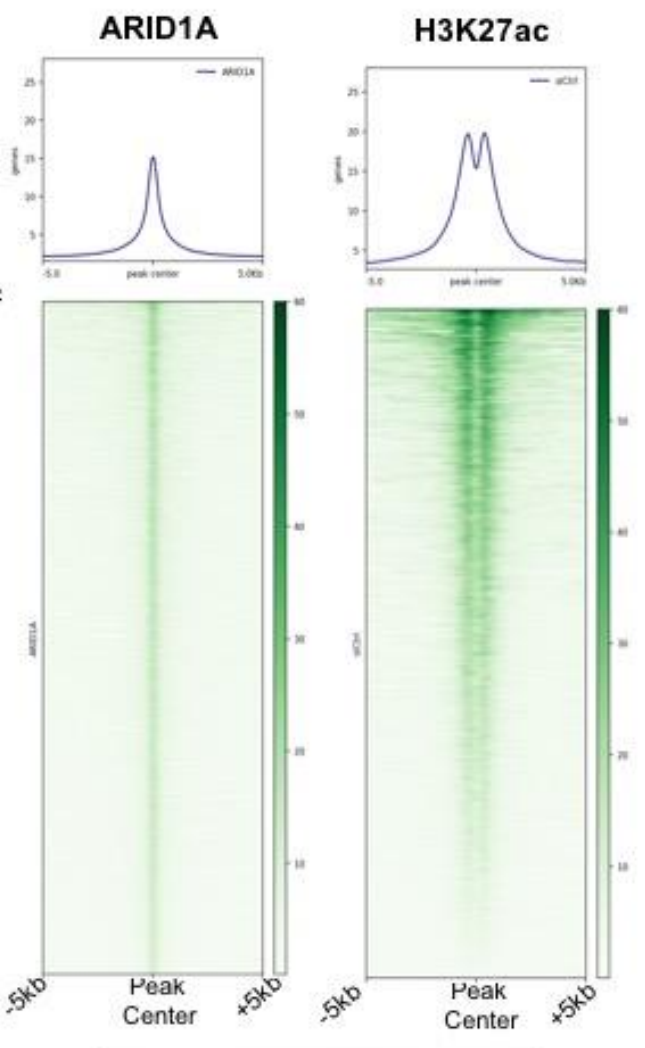

$266-6$

siArid1a+EGF

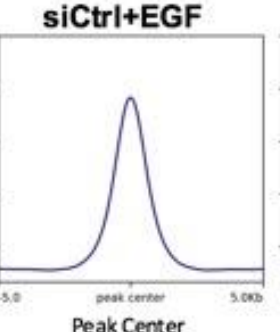

siArid1a

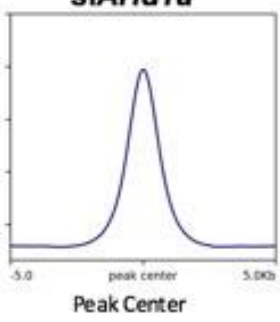

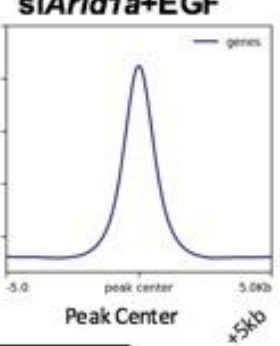

266-6 H3K27ac ChIP

Figure 17. ARID1A binds active chromatin. (A) PCA of H3K27ac ChIP-seq $(n=2)$ of the indicated conditions in 266-6 cells. (B) Average binding profiles and heatmaps depicting the H3K27ac and ARID1A occupancy across the ARID1A-occupied genomic regions. (C) Correlation plot of H3K27ac- and ARID1A ChIP-seq peaks. (D) Average profile of H3K27ac ChIP-seq in 2666 cells plotted around peak center of the indicated conditions. 
A

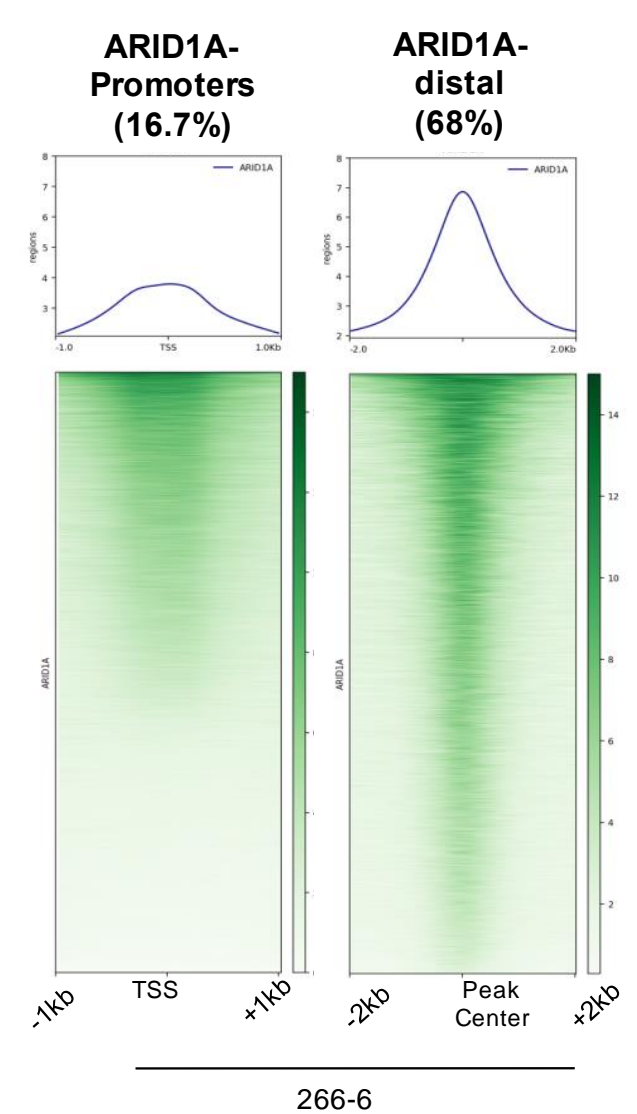

B

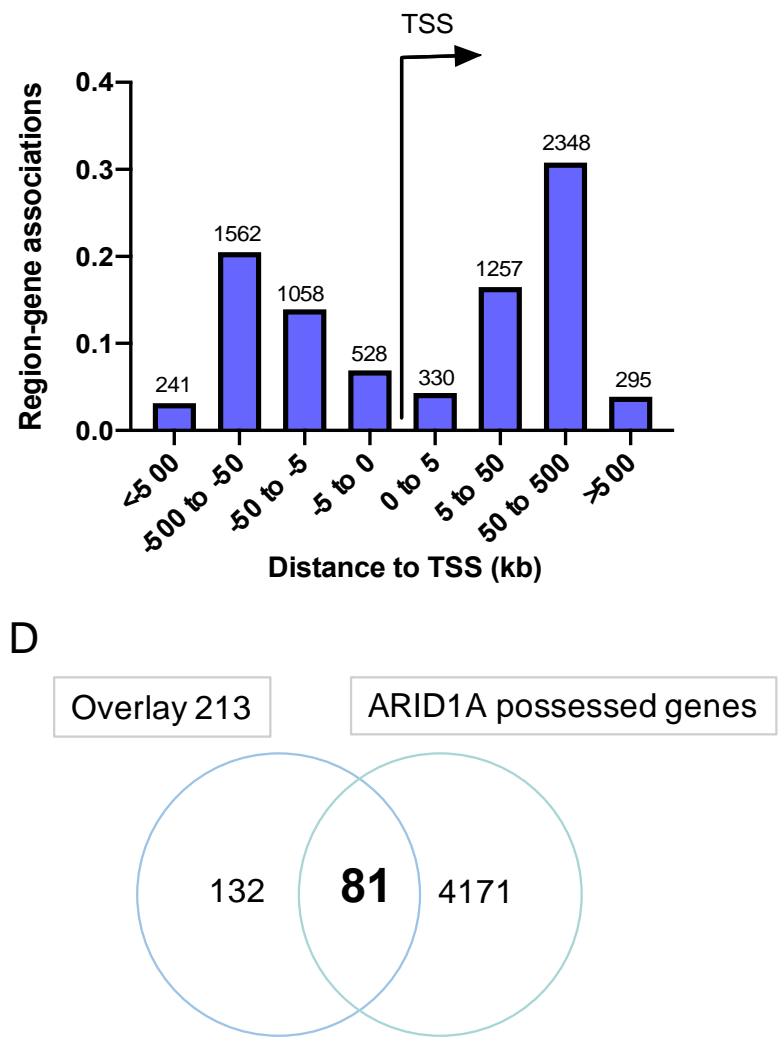

C

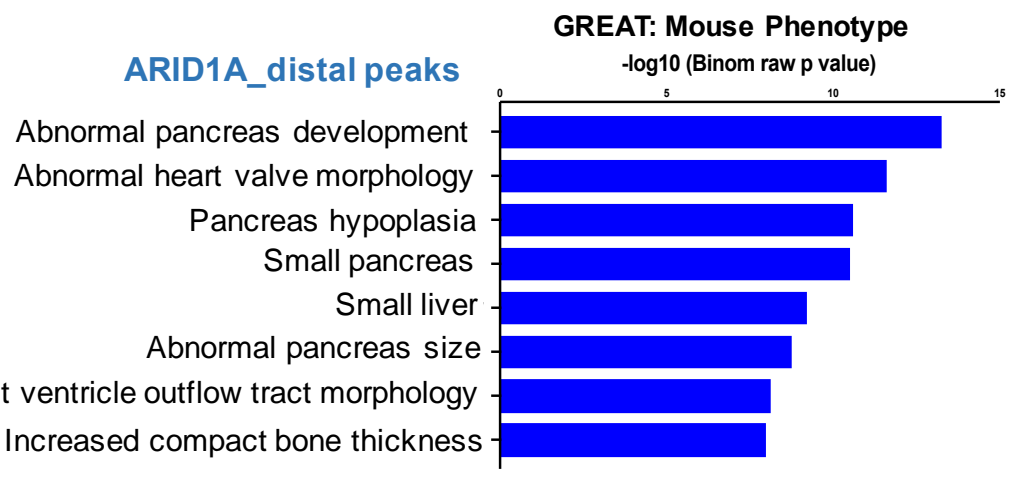

Figure 18. ARID1A binds active promoters and enhancers. (A) Average binding profiles and heatmaps of ARID1A occupancy in 266-6 cells suggest that ARID1A binds primarily active enhancers. TSS, transcription start site. (B) Bar graph from GREAT depicting the distribution of ARID1A ChIP-seq peaks as defined by distance to TSS. The analysis in GREAT was performed with single-nearest gene association rule with default settings. (C) Top-ranked terms of mouse phenotype determined by GREAT analysis of genes associated with ARID1A distal peaks. Associated genes were identified with basal plus extension association rule with default settings. The $x$-axis corresponds to the raw binomial p-values. (D) Venn diagram showing 81 out of 213 cooperatively up-regulated genes harboring ARID1A occupancy. 


\subsection{EGFR signaling activity leads to genome-wide ARID1A dissociation from the genome}

Given that EGFR signaling activity induced ductal transcription programs and facilitated an ADM phenotype even in the presence of ARID1A, we asked whether and how EGFR signaling activation affects ARID1A genomic occupancy. To this end, we conducted ChIP-seq of ARID1A following EGF treatment and compared ARID1A occupancy with its binding profile in control condition. The chromatin profiles of ARID1A in the absence and presence of EGF were clearly separated as shown by PCA (Figure 19A). Remarkably, following EGF treatment, the vast majority of ARID1A was diminished from its previous locations (Figure 19B).

A

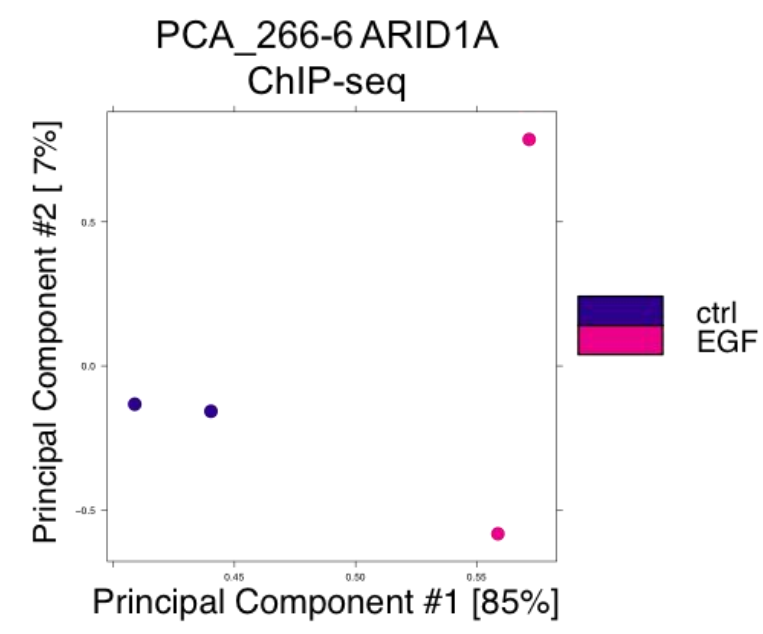

B

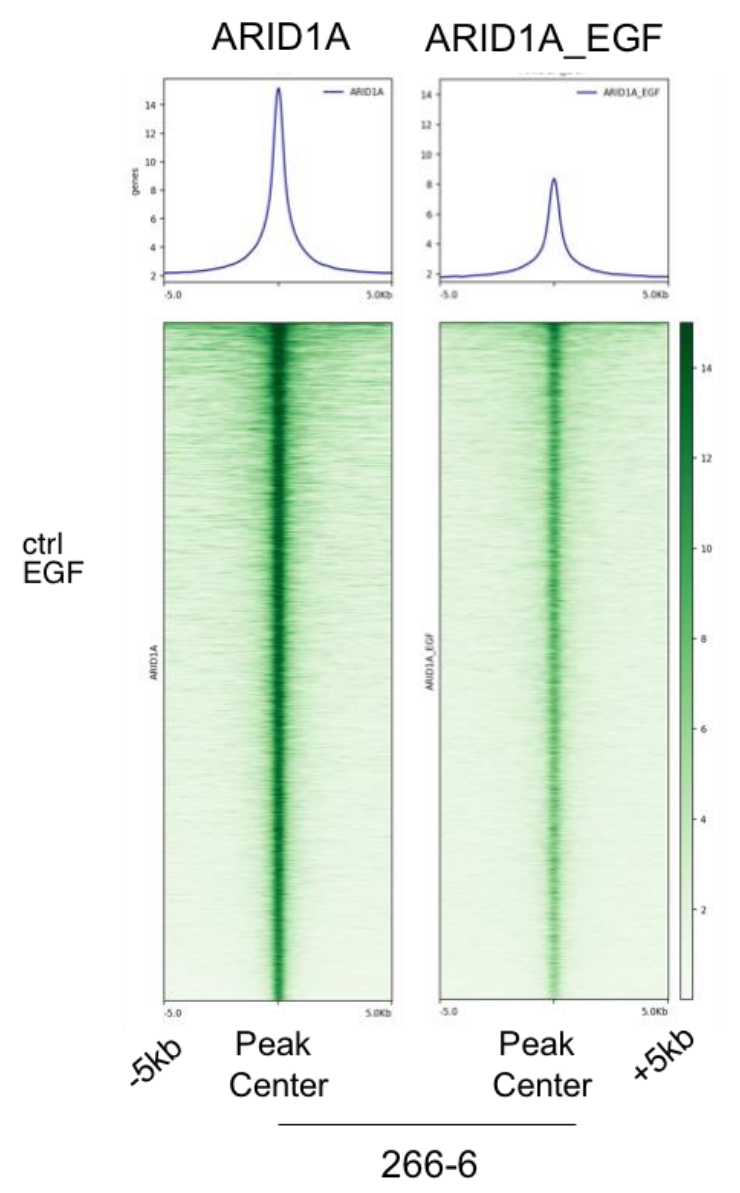

Figure 19. EGFR signaling activation reduces genome-wide ARID1A occupancy. (A) PCA of ARID1A ChIP-seq ( $n=2 /$ condition). (B) Average binding profiles and heatmaps showing the occupancy of ARID1A before and after EGF treatment as identified by ChIP-seq analysis. 
To verify this finding, we primarily excluded the possibility that the elimination of genomicbound ARID1A was led by total expression downregulation. Total ARID1A level was analyzed in whole cell lysate with increased loading dose, however, no alterations in ARID1A expression were detected upon EGF treatment (Figure 20A). We further examined ARID1A expression in different cellular fractions. As expected, ARID1A was hardly detected in cytoplasmic fraction while a large portion of ARID1A was released from chromatin into the nucleoplasm upon EGF treatment (Figure 20B). Those findings demonstrate a role of EGFR activity in influencing ARID1A-dependent transcription through regulating ARID1A genomic disposition, rather than its expression. Importantly, ARID1A disaffiliation was observed on 67 out of the aforementioned 81 ARID1A target genes which were cooperatively upregulated upon ARID1A loss and EGFR activity (Figure 20C). Among them were target genes involved in EMT, such as Bhlhe40, Fam3c and Met (Asanoma et al. 2019; Waerner et al. 2006; Jeon et al. 2017) and epithelial cell development, such as Lif and Foxa3 (Levy et al. 2010; Wangensteen et al. 2015). Tracks of ChIP- and ATAC-seq of Bhlhe40, Lif and Met are shown as examples of 67 target genes (Figure 21A-C). Alterations of H3K27ac- and ARID1A occupancies on these genes were confirmed in independent ChIP experiments (Figure 21D-E).

A

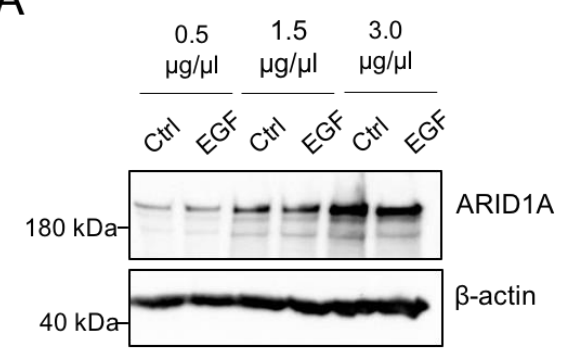

C 81 overlay

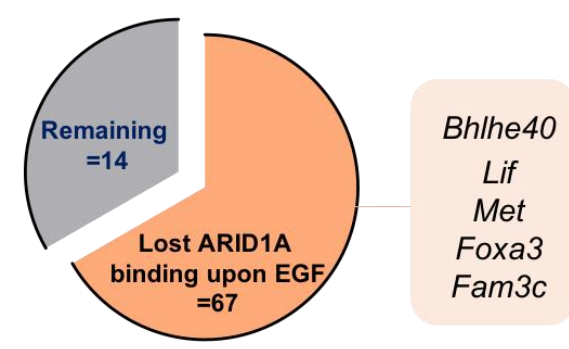

B
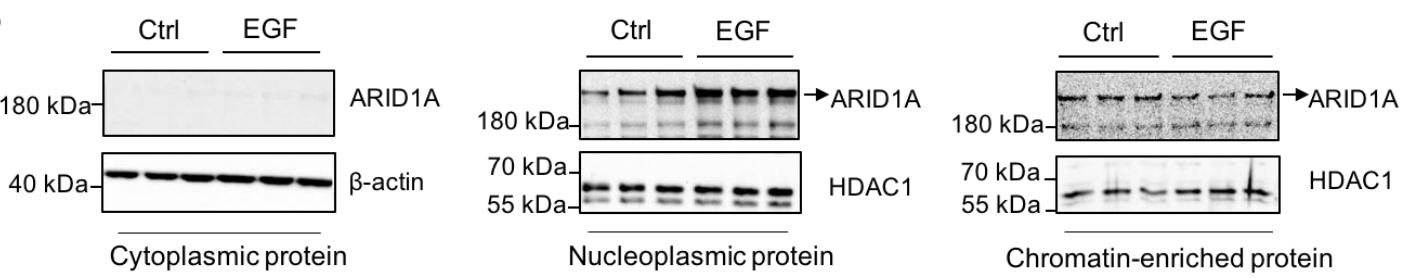

Figure 20. EGFR signaling induces ARID1A disassociation from chromatin to nucleoplasm. (A) Western blotting with different loading dosages shows that total ARID1A expression in whole cell lysates is unaffected by EGF stimulation. (B) Western blot analysis of ARID1A performed with fractionated cell lysates protein with $\beta$-actin as the loading control for cytoplasmic protein and HDAC1 for the nuclear fraction. (C) Pie chart summarizes the proportion that lose ARID1A binding (fold change $>0, F D R<0.1$ as determined by DiffBind analysis) within the aforementioned 81 ARID1A-occupied genes (shown in Figure 18D) in response to EGFR activation. Box depicts an excerpt of the final 67 target genes which lose ARID1A binding upon EGF treatment. 
A

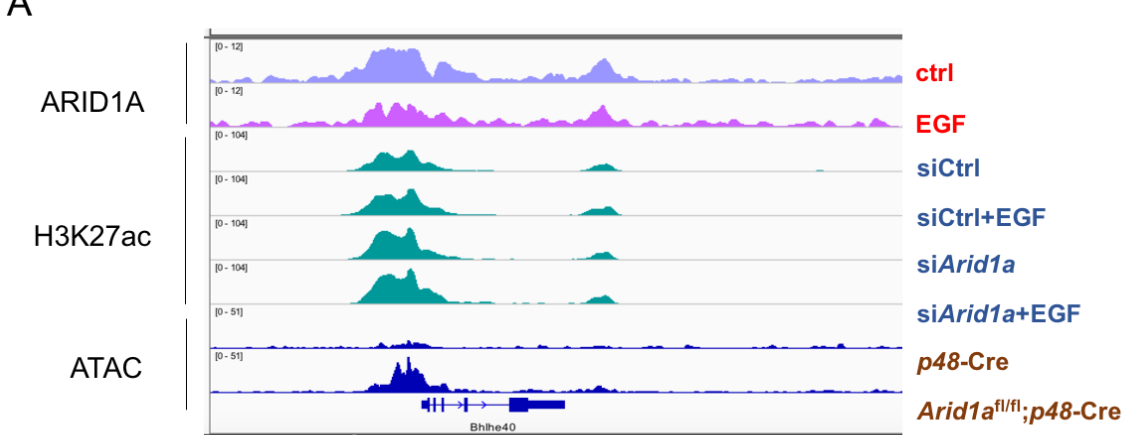

B

Bhlhe40

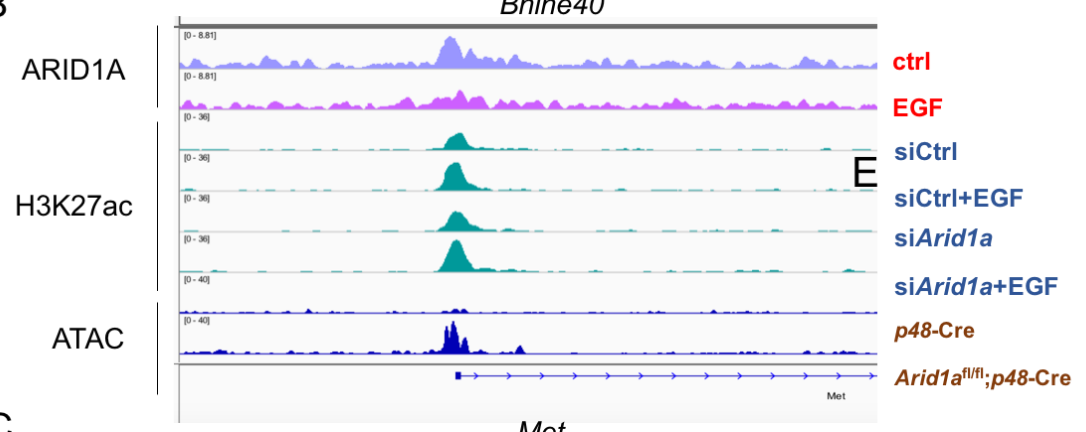

C

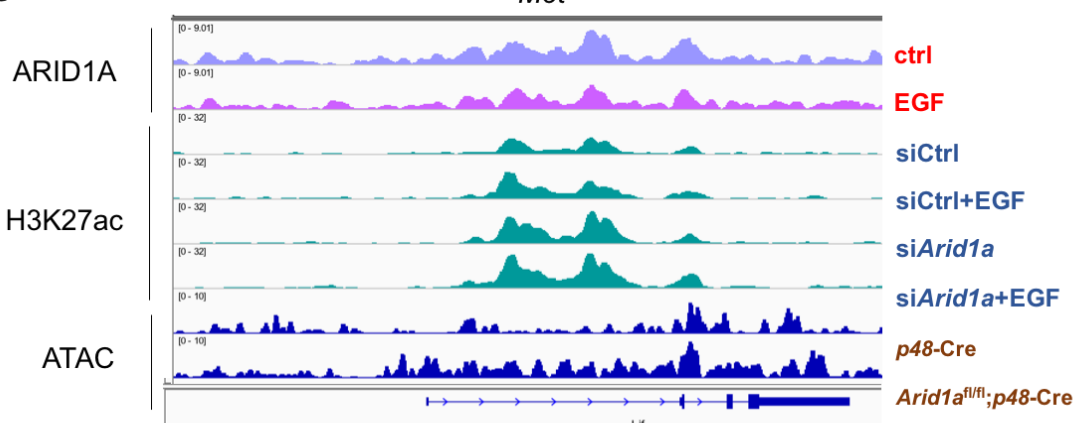

D

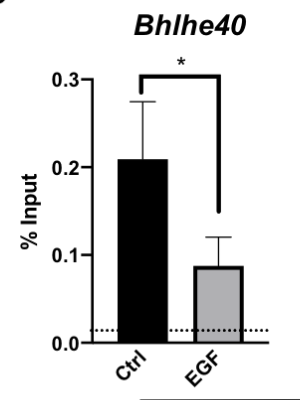

Lif
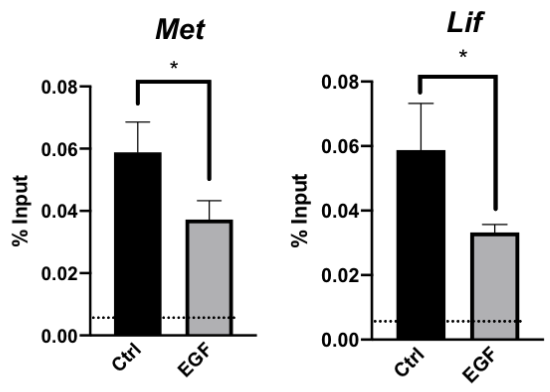

E

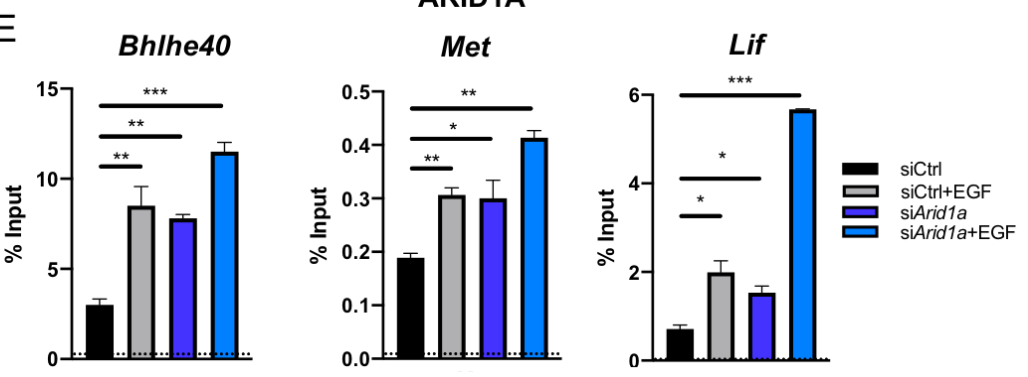

Figure 21. Tracks of ChIP- and ATAC-seq peaks of Bhlhe40, Lif and Met gene. For legend, see next page. 
Tracks of ChIP- and ATAC-seq peaks of Bhlhe40, Lif and Met gene. (A-C) Genome browser screenshots of ChIP-seq analysis (ARID1A and H3K27ac) and ATAC-seq of Bhlhe40 (A), Lif (B) and Met (C). (D-E) Individual ChIP analysis of ARID1A before and after EGF treatment (D) and $H 3 K 27 a c$ in the indicated conditions (E). Dotted line represents IgG. Data in $D$ and $E$ were presented as mean \pm s.d. Statistical significance was determined by unpaired $t$-test. $n=2 .{ }^{*} P<0.05$, ${ }^{* *} P<0.01,{ }^{* * *} P<0.001$. The validation of RNA-seq can be found in Figure $23 \mathrm{C}$.

\subsection{NFATC1 drives ADM formation in the context of Arid1a loss and EGFR signaling by displacing ARID1A from the genome}

\subsubsection{NFATc1 is required for Arid1a loss-induced acinar reprogramming}

Given that cellular identity are initiated during development with particular transcriptional programs which are under control of transcription factors (Baumann et al. 2019), we hypothesized that transcription factors might be causally involved in the genome-wide ARID1A displacement upon EGFR signaling activation. Therefore, we performed motif discovery via HOMER, searching for transcription factors that bind on ARID1A-occupied genomic regions. The regulatory sites of ARID1A harbored a variety of binding motifs for developmental transcription factors such as FOXA2, GATA4 and NR5A2 (Figure 22A). Interestingly, one of the most enriched motifs was the consensus site (GGAAA) for Nuclear Factor of Activated T-cells (NFAT). The NFAT family of transcription factors respond to $\mathrm{Ca}^{2+}$ influx and translocate into the nucleus after being dephosphorylated by the serine/threonine phosphatase calcineurin (Mancini et al. 2009). Besides the impact of inducing ADM, NFATc1 was verified as a downstream target of EGFR signaling (Chen et al. 2015; Chen et al. 2017). Accordingly, pharmacological or genetic disruption of NFATC1 has been shown to block inflammation-driven pancreatic carcinogenesis (Chen et al., 2015; Hasselluhn et al., 2019). Thus, we hypothesized that NFATc1 might participate in the transcriptional activation of ductal genes upon EGFR-signaling and in the context of Arid1a deficiency. To verify this, we overlapped NFATc1-induced genes with ARID1A bound genes and the ones that were transcriptionally activated by Arid1aknockdown and EGFR activation and observed a considerable number of 262 genes fulfilling these conditions (Figure 22B). Using ChIP-qPCR, we confirmed the enrichment of NFATc1 on a subset of direct ARID1A target genes (Figure 22C).

To investigate whether Arid1a loss has an impact on NFATc1 activity, we conducted a reporter assay in 266-6 cells by employing a luciferase construct containing an NFATresponsive promoter. In line with previous findings (Chen et al. 2015), we observed a 
prominent increase of luciferase activity upon EGFR overexpression. Meanwhile, a comparable level of transactivation was found upon Arid1a-knockdown, suggesting an analogous role of EGFR signaling and Arid1a deficiency in inducing NFAT transactivation (Figure 22D).

A

\begin{tabular}{|c|c|c|c|}
\hline $\begin{array}{l}\text { Transcription } \\
\text { Factor }\end{array}$ & Motif & $\begin{array}{l}\text { \%targets with motif } \\
\text { (\%bg with motif) }\end{array}$ & $p$-value \\
\hline FOXA2 & TGTTACACA & $15.14 \%(5.82 \%)$ & $1.00 \mathrm{E}-758$ \\
\hline TCF21 & AACACCTGCC & $22.72 \%(12.06 \%)$ & $1.00 E-593$ \\
\hline GATA4 & 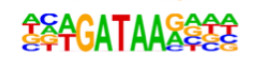 & $18.85 \%(10.66 \%)$ & $1.00 E-397$ \\
\hline ELK1 & 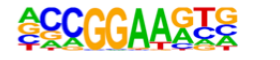 & $11.50 \%(6.25 \%)$ & $1.00 E-258$ \\
\hline NFAT & ТCTCATTTCCA & $31.52 \%$ (23.49\%) & $1.00 \mathrm{E}-226$ \\
\hline NR5A2 & C्C्रCAAGGICA & $5.22 \%(2.20 \%)$ & $1.00 E-209$ \\
\hline
\end{tabular}

C

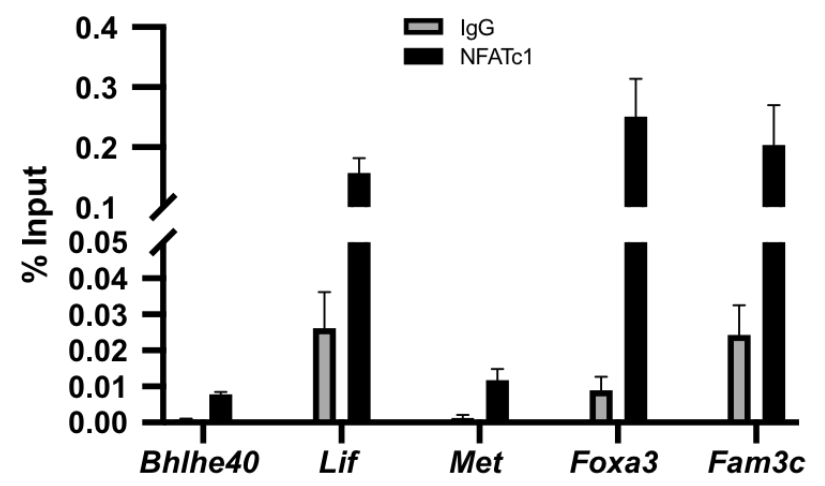

B

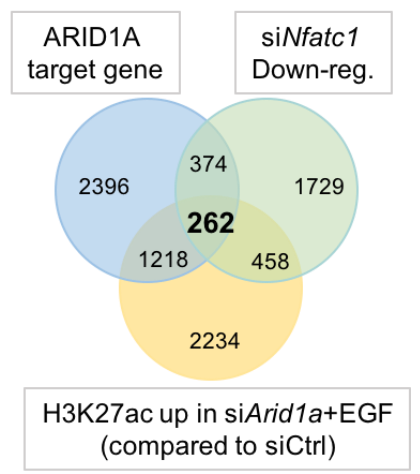

cisNFAT-luc

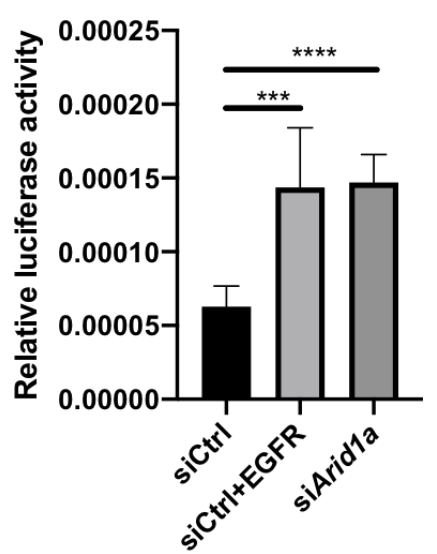

Figure 22. NFATc1 binds a subset of ARID1A target genes and is trans-activated by Arid1aknockdown. (A) Transcription factor motifs (identified by HOMER) enriched at ARID1A binding genomic regions as detected by ChIP-seq peak calling (fold enrichment $>5$ compared to background, FDR<0.05). (B) Venn diagram showing the overlap of genes which are bound by ARID1A (FDR $\leq 0.05$ ), down-regulated by NFATc1 depletion (log2 fold change $<0, q \leq 0.1$ ) as identified by RNA-seq analysis conducted in pancreatic cancer cells (Hasselluhn et al. 2019), and increased H3K27ac enrichment (log2 fold change<0, $\mathrm{q} \leq 0.05$ ) upon EGFR-activation in the absence of Arid1a. Associated genes of ARID1A and H3K27ac peaks were identified by GREAT with basal plus extension association rule and default setting. (C) ChIP-analysis in 266-6 cells and subsequent qPCR confirmed the occupancy of NFATc1 on the direct ARID1A target genes Bhlhe 40, Lif, Met, Foxa3 and Fam3c in the presence of EGF. (D) Luciferase reporter assay of an NFAT-responsive promoter construct in cells overexpressed with EGFR or upon Arid1aknockdown. Data is representative for three independent experiments. Data in $D$ are presented as mean \pm s.d.. Statistical significance was determined by two-tailed unpaired Student's $t$-test. ${ }^{* * *} P<0.001,{ }^{* \star *} P<0.0001$. 
Next, we sought to test if NFATc1 activation influences Arid1a-loss-induced ductal gene activation and ADM formation. Consistent with the previous study (Chen et al. 2017), constitutive activation of NFATc1 triggered acinar transdifferentiation in vitro, which is comparable to the Arid1a-deficient phenotype (Figure 23A). Next we employed cyclosporine A (CsA), which blocks NFATc1 activity, for ADM culture in the context of EGF treatment or/and Arid1a-knockdown. The successful NFATc1 inhibition was confirmed by western blot by blockade of NFATc1 nuclear translocation (Figure 24C) and Arid1a knockdown was verified by qRT-PCR (Figure 23B). Notably, CsA treatment significantly suppressed the ARID1A target genes upregulation led by EGFR signaling activation and Arid1a-knockdown and impaired acinar-to-ductal conversion (Figure 23 C-D). 
A

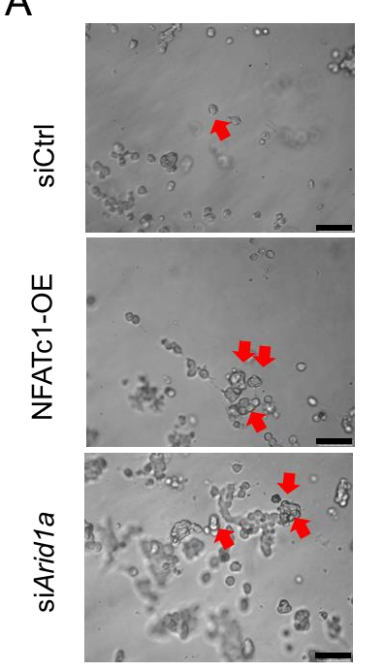

C

D

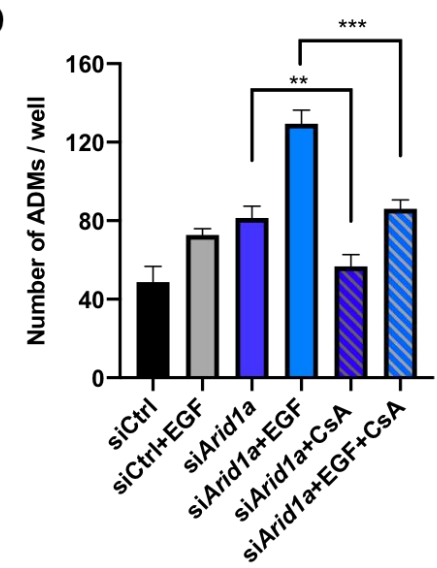

B
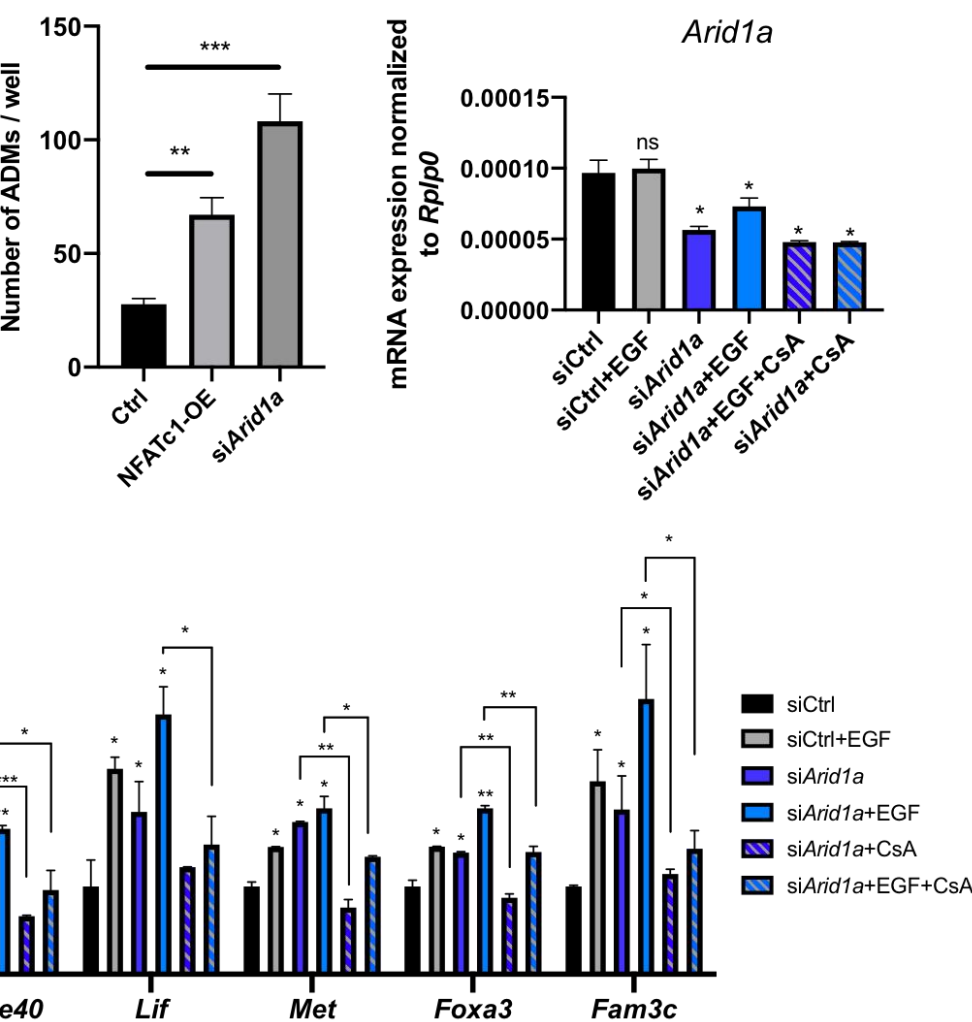

Figure 23. NFATc1 activity promotes ADM formation in the context of Arid1a deficiency. (A) Representative images (left) and quantification (right) of ADM formation following NFATc1 overexpression and Arid1a-knockdown in 266-6 cells conducted at day 4. Red arrowheads point to the duct-like structures. Scale bars: $50 \mu \mathrm{m}$. (B) qRT-PCR analysis to confirm successful Arid1aknockdown in 266-6 cells for experiments shown in Fig. 22D. (C) Gene expression analysis of indicated genes following individual- or combined EGF or CsA treatment in Arid1a-depleted 2666 cells. Data are shown as relative mRNA expression normalized to Rp/pO housekeeping gene. (D) Duct formation was determined in 266-6 cells upon Arid1a-knockdown following in the presence or absence of EGF or/and CsA treatment. Quantification was performed at the fourth day after seeding. Data in $C$ and $D$ are presented as mean \pm s.d.. Statistical significance was determined by two-tailed unpaired Student's $t$-test. ${ }^{*} P<0.05$, ${ }^{* *} P<0.01,{ }^{* * *} P<0.001$. 


\subsubsection{NFATc1 disposes ARID1A from its target genes}

Given that EGFR-mediated NFATc1 activation is sufficient to induce ductal transcriptional programs and promote ADM formation even in the absence of Arid1a-ablation (Chen et al., 2017), we proposed that EGFR-induced NFATc1 activation is involved in ARID1A dissociation from the genome. To explore this hypothesis, we correlated the aforementioned ARID1A target genes (Figure 20C) with NFATc1-induced genes. 13 out of $67(19 \%)$ genes that lost ARID1A upon EGF treatment were NFATc1 targets while only 1 out of $14(7 \%)$ of ARID1A remaining sites fell in this category, suggesting that NFATc1 is more likely to target those sites that lose ARID1A occupancy upon EGF treatment (Figure 24A). To examine if NFATc1 is sufficient to dispose ARID1A from chromatin, we performed cellular fractionation assays with cells overexpressing constitutive active NFATc1. Western blot analysis conducted with whole cell lysates confirmed the overexpression of NFATc1 as well as the stable expression of ARID1A (Figure 24B). Interestingly, overexpressed NFATc1, which was aggregated in the nucleus, caused ARID1A translocation from chromatin to nucleoplasm (Figure 24C). In parallel, we also examined ARID1A expression in different cellular fractions upon CsA-induced blockade of NFATc1 activity. CsA treatment did not affect total NFATc1 expression, but did reverse its nuclear translocation by showing a substantial portion of NFATc1 in the cytoplasmic fraction (Figure 25B). Meanwhile, total ARID1A level remained unchanged upon CsA (Figure 25A), which, however, caused striking accumulation of ARID1A on chromatin (Figure 25B). More specifically, we detected the occupancy of ARID1A on its target genes under the impact of NFATc1 activity by performing ARID1A ChIP-qPCR upon CsA treatment. NFATc1-inhibtion-induced restoration of ARID1A on its target genes (Figure 25C). Hence, we reason that EGFR-dependent NFATc1 activation and its subsequent nuclear translocation is essential for ARID1A genomic dissociation, thus promoting the transcriptional activation of gene signatures favoring ductal program. 
A

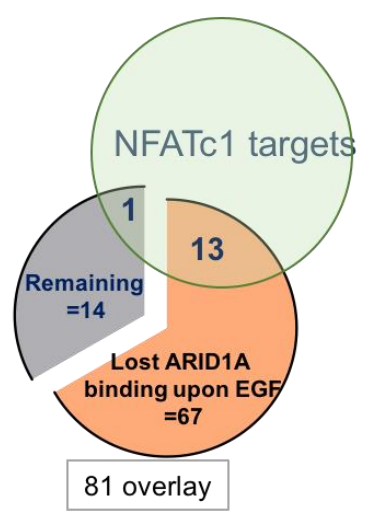

B

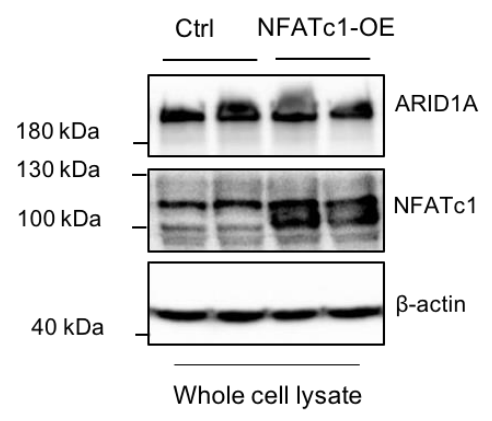

C
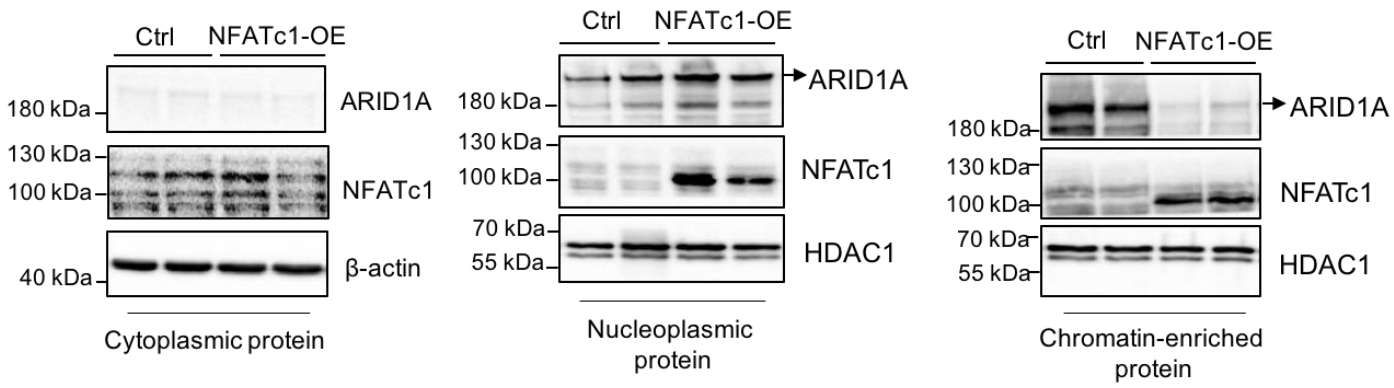

Figure 24. NFATc1 determines genomic allocation of ARID1A. (A) Venn diagram showing the overlapped proportion between NFATc1-induced genes (Hasselluhn et al. 2019) and previouslydescribed 81 genes with remaining or diminished ARID1A binding upon EGF treatment. (B) Western Blot analysis conducted with whole cell lysates of 266-6 cells upon NFATc1 overexpression. (C) The expression of NFATc1, ARID1A in different cellular fractionation lysates was analyzed by immunoblotting in cells upon NFATc1 overexpression. $\beta$-actin was used as the loading control for cytoplasmic protein and HDAC1 for the nuclear protein. 


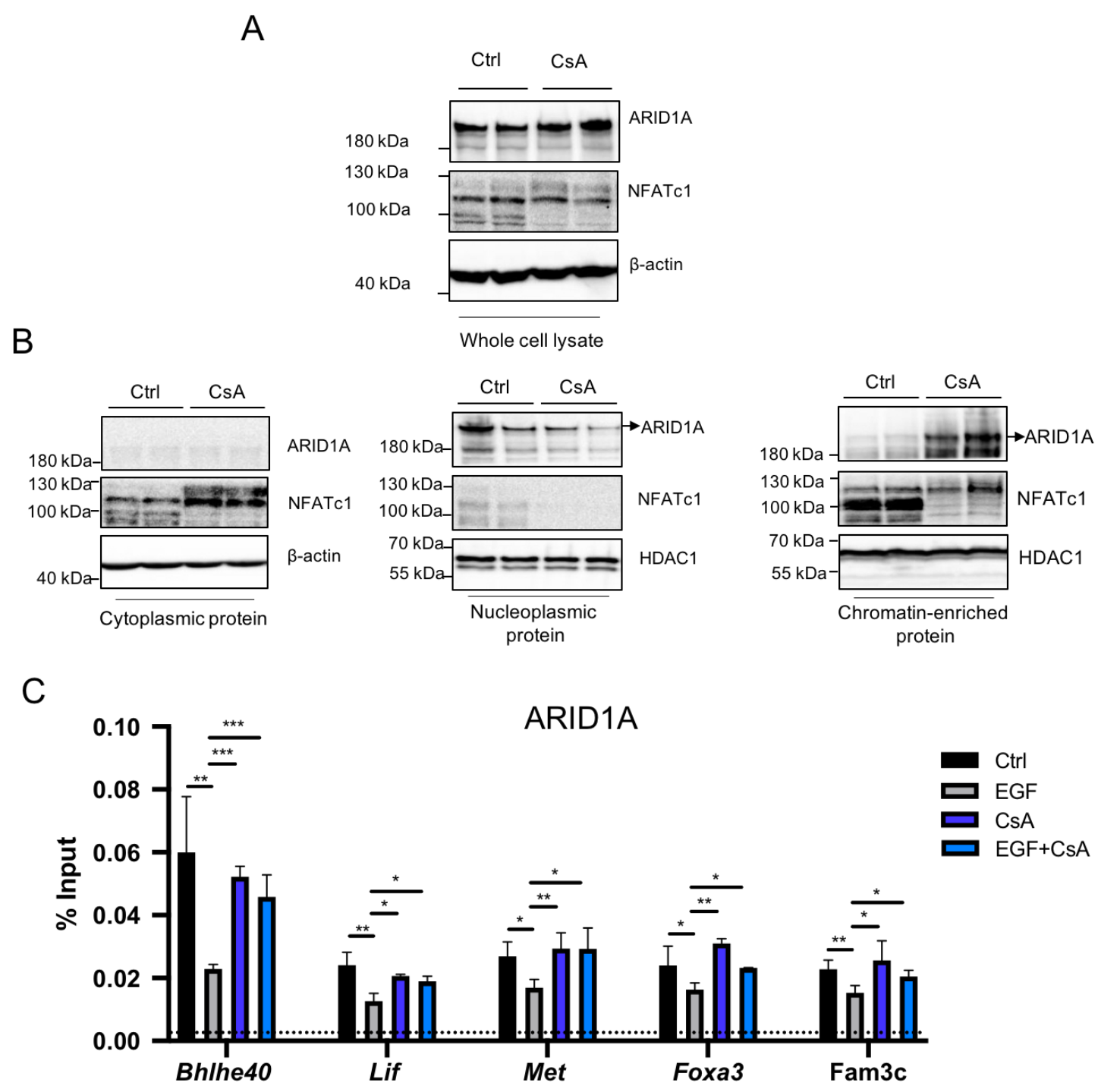

Figure 25. NFATc1 determines genomic allocation of ARID1A. (A) Western Blot analysis conducted with whole cell lysates of 266-6 cells upon CsA treatment. (B) The expression of NFATc1, ARID1A in different cellular fractionation lysates was analyzed by immunoblotting in cells upon CsA treatment. $\beta$-actin was used as the loading control for cytoplasmic protein and HDAC1 for the nuclear protein. (C) ARID1A ChIP analysis and subsequent qRT-PCR on indicated genes in the presence and absence of EGF and CsA. Dotted line represents IgG levels. Data was presented as mean \pm s.d. $(n=2)$ and two-tailed unpaired Student's $t$-test was used to calculate statistical significance. ${ }^{*} P<0.05,{ }^{* *} P<0.01,{ }^{* * *} P<0.001$. 
Page | 74 


\section{Discussion}

\subsection{The role of ARID1A in modulating pancreatic acinar cell plasticity}

Pancreatic epithelial cell plasticity is frequently associated with development and increased risk of neoplasia (Stanger and Hebrok 2013). The conversion between differentiation states, as reflected by a diminution of the original phenotype and an acquisition of characteristics of another cell type, is under the influence of various physiological or pathological perturbations. Lineage tracing studies conducted by Kopp et al. suggested acinar cells, but not ductal or centroacinar cells, as the predominant origin of Kras-driven PanIN pre-neoplastic lesions (Kopp et al. 2012). These findings emphasize the importance of maintaining acinar cell differentiation as a strategy against neoplastic transformation. ARID1A has been found to be essential in maintaining the differentiation state of various cell types, including adipocytes, hepatocytes and cardiomyocytes (Lei et al. 2012; Sun et al. 2017; Giri et al. 2018). Studies in the pancreas revealed the commitment of ARID1A in pancreas development and acinar cell homeostasis (Wang W. et al. 2019; Wang S. C. et al. 2019; Kimura et al. 2018; Livshits et al. 2018). Pancreas-specific Arid1a loss in transgenic mice leads to decreased acinar cell mass, PanIN formation and inflammation infiltration while concurrent Kras activation cooperates with Arid1a deficiency to generate IPMN and carcinogenesis (Wang W. et al. 2019; Wang S. C. et al. 2019; Kimura et al. 2018; Livshits et al. 2018). Gene sets enrichment analysis correlates Arid1a loss leads to induction of genes associated with Myc activity (Wang S. C. et al. 2019) and EMT (Wang W. et al. 2019). Consistent with the previous findings, our study demonstrates that Arid1a loss is sufficient to drive duct dilation as well as ADM and PanIN formation at early age of mice, even in the absence of oncogenic Kras. Heterozygous loss of ARID1A also induces ductal lesions in mice, but only after longer latency (> 1 year) and with reduced frequency and severity. In addition, the intrinsic capability of Arid1a-null cells in inducing acinar cell transdifferentiation is confirmed by in vitro acinar explant assays. In contrast to control cells, acinar cells with heterozygous- or homozygous- Arid1a loss are capable of converting into ductal-like structures which based on their morphology and marker gene expression can be identified as ADM lesions. Thus, by providing a potent barrier for acinar-to-ductal transdifferentiation, ARID1A is necessary in preserving the terminal differentiation state of the exocrine pancreas.

Remarkably, high grade PanIN or neoplastic tissues could never be detected in Arid1a 
deficient mice, implying that Arid1a loss in the absence of oncogenic KRAS may not be sufficient for the transition to high grade PanIN and further stages. However, the mice harboring biallelic Arid1a depletion become moribund at an average age of 2.6 months, which is more rapid than the mice with $K_{r a s}^{G 12 D}$ mutation (O'Hagan et al. 2011). We noticed that the moribund Arid1a-knockout mice, without exception, bear maldigestioncaused gut blockage, which might account responsible for the pain symptoms, these mice develop. Accordingly, Livshits et al. show that mice with acute Arid1a-knockdown exhibit steatorrhea (Livshits et al. 2018), which, together with our finding, suggests a phenotype of exocrine insufficiency which leads to the lethality of Arid1a deficiency.

The conversion of the acinar to a ductal-like phenotype is ensured by a transcriptional shift from acinar markers to genes characterizing the ductal state. Several basic helixloop-helix $(\mathrm{bHLH})$ transcription factors contribute to acinar cell identity including pancreas associated transcription factor 1 (PTF1). PTF1 drives the specification of the acinar lineage and regulates digestive enzymes production (Dong et al. 2008). The depletion of Ptf1 is sufficient to provoke ADM (Johnson et al. 2012). Our RNA-seq analysis correlates Arid1a loss with PanIN- and inflammation- promoting pathways. Notably, the genes that associate with Ptf1a-deficient phenotypes are also found enriched in acinar cells with Arid1a loss, suggesting an intersection between the regulation networks of ARID1A and other well-known acinar markers in maintaining acinar cell integrity. 


\subsection{Arid1a loss alters the epigenomic landscape}

Although SWI/SNF complexes have been shown to regulate gene transcription since two decades ago (Kowenz-Leutz et al. 1999), the precise mechanism by which different subunits participate in this process remains obscure. As a master regulator of development, SWI/SNF predominantly binds lineage-determining genes and the conjugations are found both at promoters and enhancers (Tolstorukov et al. 2013; Hu et al. 2011; Bossen et al. 2015). Especially in recent years, the regulation of SWI/SNF on genomic enhancers has been brought under spotlight. In embryonic stem cells, mutations of the catalytic subunit BRM results in enhancer reprogramming (Gao et al. 2019). In breast cancer cells, ARID1A directly binds and represses enhancers that have estrogen receptor binding elements (Nagarajan et al. 2020). In colorectal cancer, Sen et al. showed that ARID1A localizes to AP1-bound enhancers (Sen et al. 2019) Here, we provide a genome-wide profiling of the SWI/SNF subunit ARID1A in pancreatic acinar cells. Consistent with several studies which suggest an enhancer-preferential binding manner of ARID1A (Mathur et al. 2017; Kelso et al. 2017), 68\% of the ARID1A peaks in our model are detected in distal regions that are marked by H3K27ac while only $16.7 \%$ are located at TSS-proximal regions. The distal ARID1A peaks, as given by gene ontology analysis, confirm the critical lineage-specific regulation of ARID1A in pancreas development and morphogenesis. Further analysis can be done to verify the enhancer property of these distal peaks of ARID1A, by detecting the co-localization with H3K4me1 and general coactivators (such as p300/CBP), low levels of H3K4me3 promoter-specific marker and determining enhancer RNA (eRNA) expression (Kim et al. 2015).

SWI/SNF is believed to function predominantly in facilitating transcriptional activation, Arid1a loss leads to generally decreased H3K27 acetylation (Trizzino et al. 2018; Sen et al. 2019). Unlike these literatures, we found that global H3K27ac level is increased upon Arid1a knockdown and sites which gain H3K27ac are mostly those which were bound by ARID1A previously, suggesting a distinct role of ARID1A other than being a strong transcriptional activator. Similar findings were reported in human embryonic stem cells (hESC) in which SMARCB1 has a repressive role at enhancers regions (Langer et al. 2019) and in breast cancer where ARID1A interacts with HDAC1, Arid1a depletion leads to substantial decrease of HDAC1 binding and subsequent shift in acetylation status (Nagarajan et al. 2020). Remarkably however, in our study, we observed that the vast majority of ARID1A peaks co-localize with the transcriptional active mark H3K27ac, yet there has been no evidence of ARID1A being a transcriptional repressor. Given the 
augmentation of $\mathrm{H} 3 \mathrm{~K} 27 \mathrm{ac}$ upon Arid1a loss, we hypothesized that ARID1A prevents hyper-acetylation of its bound regions. Interestingly, Wilson et al. supported this model with the finding that super-enhancers which are marked by H3K27ac are strongly associated with ARID1A occupancy in endometrial epithelia, ARID1A loss leads to hyperacetylation and enhancer-RNA transcription at these super-enhancer regions (Wilson et al. 2020). Chromatin remodeling enzymes regulate recruitment as well as the catalytic activity of histone modifying enzymes, such as acetyltransferases (Clapier and Cairns 2009). Previous studies have detected physical interactions between the acetyltransferase P300 and SWI/SNF subunits SMARCA4, SMARCC1 and SMARCB1(Ogiwara et al. 2011; Huang et al. 2003; Alver et al. 2017), as well as ARID1A (Wilson et al. 2020). The hyper-acetylation of super-enhancers led by ARID1A loss is under mediation of P300 (Wilson et al. 2020). Being as the factor that displaces ARID1A from its target genes, NFATc1 is also found with the capacity to interact with p300 and initiate histone acetylation of $c-M y c$ (Köenig et al. 2010). These results collectively suggest that the increase of H3K27ac seen in our case might be due to p300 regulation by Arid1a loss or NFATc1 activity.

The increased H3K27 acetylation and subsequent reprogramming of transcription significantly promote the phenotypes determined by ARID1A loss. For instance, P300mediated super-enhancer hyper-acetylation is required for the invasion capacity of ARID1A deficient endometriotic cells (Wilson et al. 2020). Accordingly, interfering with H3K27 acetylation might represent a promising therapeutic strategy to interfere with pathological cellular phenotypes installed in the context of ARID1A loss. A-485 is a smallmolecule inhibitor for P300/CBP histone acetyltransferase (Lasko et al. 2017). Invasion of the ARID1A-deficient endometriotic cells was strikingly blocked by A-485 treatment (Wilson et al. 2020). Apart from the acetyltransferases, another family of proteins which are "readers" of histone acetylation marks also become target for blockade of acetylation signaling. Bromodomain and extraterminal (BET) proteins, including BRD2, BRD3, BRD4 and BRDT, recognize lysine acetylation and translate the signal carried by the acetylation mark into cellular processes (Ntranos et al. 2016). JQ1 is the first drug discovered to interact with BET-bromodomain hydrophobic pocket thus to block interaction between BRD 2/3/4 and acetylated histones (Filippakopoulos et al. 2010) and has been shown to efficiently block various tumor growth (Belkina et al. 2013; Shin et al. 2018; Delmore et al. 2011). In our model, JQ1 treatment effectively slowed down the acinar-to-ductal transdifferentiation led by Arid1a-knockdown (data not shown), partly 
confirming the feasibility of inhibiting H3K27ac signal transduction in Arid1a-null context as a therapeutic strategy.

Extensive literatures based on in vitro studies have shown that ARID1A-mediated chromatin remodeling, by ensuring the proper expression of key differentiation factors, is an essential epigenetic mechanism for development and lineage commitment (Lei et al. 2012; Gao et al. 2008). In line with the previous studies, we found that loss of acinar identity is accompanied by widespread transcriptome changes, thus chromatin accessibility could be informative of these altered transcriptional states. ATAC-seq reveals that ablation of ARID1A indeed results in striking alterations of the chromatin accessibility, with substantially more open regions detected in cells lacking ARID1A. The majority of the sites with enhanced accessibility are 5'UTR and TSS-proximal promoter regions, which are of central importance for transcriptional regulation. The subset of genes that gain accessibility in ATAC-seq are positively correlated with the significant changes in transcriptional activity seen in RNA-seq, highlighting the importance of DNA accessibility in influencing gene expression. Although ARID1A-containing SWI/SNF complexes are considered to serve as maintainer of chromatin accessibility, studies show distinct roles of ARID1A in regulating chromatin structures in different developmental background (Kelso et al. 2017; Livshits et al. 2018). Global alterations of chromatin remodeling are detected both in colorectal carcinoma cells and embryonic stem cells upon ARID1A knockout, with a significant number of genes of different developmental functions either gain or lose accessibility (Kelso et al. 2017; Liu et al. 2020). Livshits et al. showed an overall reduced accessibility by Arid1a depletion in pancreatic acinar cells, which are, however, with oncogenic Kras mutation, indicating that the remodeling activity of ARID1A is highly context-dependent and can be affected by genetic events.

As for the reason behind the increased chromatin accessibility led by Arid1a loss, we surmise that other chromatin remodeling complexes or transcription factors might be involved in this process of regulation. The activator protein 1 (AP-1) transcription factor complex has been shown by previous reports to co-localize with SWI/SNF and is verified in vitro to directly bind to SWI/SNF subunit BAF60A (Mathur et al. 2017; Morris et al. 2014; Ito et al. 2001; Sen et al. 2019). Notably, AP-1 can prime chromatin followed by recruiting other transcription factors, and can potentiate chromatin accessibility (Biddie et al. 2011). Kelso et al. found an enrichment of AP-1 at the ARID1A-dependent 
accessible regions in colorectal cancer cells, which raises the possibility of the involvement of AP-1 in ARID1A-mediated chromatin regulation. In addition to transcription factors, chromatin regulatory proteins which determine histone acetylation also have impact on high-order chromatin architecture (Görisch et al. 2005). Histone deacetylases (HDACs) remove the acetyl-groups and promote compaction of the chromatin (Marks et al. 2001). Literatures have demonstrated that Arid1a deficiency impairs the recruitment of HDACs to the genome (Kim et al. 2016; Nagarajan et al. 2020). Subsequently, the HDAC-mediated repression of chromatin and transcription network is eliminated, which might be an alternative mechanism of accessibility increase led by Arid1a loss.

\subsection{The activity of the residual SWI/SNF complex upon inactivation of individual subunit}

One interesting question underlying the observations of our study is whether Arid1a deficiency leads to loss of function of the entire SWI/SNF complex or the residual complex still functional and impacts on the epigenetic alterations observed herein as well as on the ADM-permissive phenotype.

Although cases of inactivation mutations of SWI/SNF subunits are commonly seen, the functional activity of the residual complex constitutes remains largely elusive. Given that the inactivation of SNF5 and BRG1 both lead to early embryonic lethality, KlochendlerYeivin and colleagues have proposed that mutations of different SWI/SNF subunits have equivalent effects that all result in loss-of-function of the entire SWI/SNF complex (Klochendler-Yeivin et al. 2000). However, this view has been challenged by cumulative evidences. First, inactivation of different SWI/SNF components leads to distinct consequences in development. For instance, Smarcd3 silencing in mouse embryos causes defects in heart morphogenesis (Lickert et al. 2004), Smarca4 homozygous inactivation leads to early embryonic lethality whereas Smarca2-deficient mice are viable (Wilson et al. 2014). Second, the preference of SWI/SNF mutations varies with different cancer types. For example, PBRM1 is frequently mutated in renal cancer (Varela et al. 2011), ARID1A mutation occur with high frequency in endometriosis-related ovarian cancer (Takeda et al. 2016) and SMARCA4, in lung cancer (Reisman et al. 2003). Third, cells with BRG1-, SNF5- or ARID1A- mutations develop dependencies on other complex 
subunits which substitute the missing subunit and subsequently retain the function of the residual complex (Wang et al. 2009; Wilson et al. 2014; Helming et al. 2014). For instance, BRM is essential for the growth of tumor cells with loss-of-function of BRG1 and ARID1A-mutant cancer cells are particularly dependent on ARID1B activity (Hoffman et al. 2014; Helming et al. 2014). Similarly, the carcinogenesis of SNF5-null cells is not due to SWI/SNF dysfunction either, but rather depends on the BRG1 subunit in the residual complex (Wang $X$ et al. 2009).

Whether or whether not the ARID1A-deficient SWI/SNF complex remains intact in our system, remains elusive. Nevertheless, the possibility exists that other substitutable SWI/SNF subunits, for example ARID1B, is conferred with enhanced capability in the context of $A R I D 1 A$ deficiency to recruit transcription factors or co-factors for maintaining the chromatin accessibility as well as transcriptional activation.

\subsection{EGFR signaling in pancreatic cancer initiation and progression}

\subsubsection{Arid1a deficiency cooperates with EGFR signaling in driving pancreatic acinar cell programming}

The fact that KRAS mutations are detected in more than $90 \%$ of PDAC cases characterizes mutant KRAS as one of the most prominent driving genes of this cancer type. The Kras ${ }^{G 12 D}$ expression in murine acinar cells leads to ADM formation and irreversibly lock the acinar cells in a transdifferentiated duct-like state. Strikingly however, $K_{r a s}{ }^{G 12 D}$ alone does not guarantee tumor formation. Even with the universal transgenic $K$ Kas $^{G 12 D}$ expression during early organogenesis in mice, only a small portion of cells stochastically generate pre-neoplastic lesions but rarely progress into PDAC, and a long latency is needed if they do (Hingorani et al. 2003). This observation, together with the finding that oncogenic KRAS mutations are also detected in human tumor-free pancreas, suggest the requirement of additional events driving PDAC development.

Examination of human PDAC samples revealed a high frequency of upregulation of epidermal growth factor receptor (EGFR) as well as its ligands EGF and TGFa (Tobita et al. 2003). EGFR signaling plays a fundamental role in regulating the proliferation and differentiation of epithelial cells, including the exocrine pancreas. Normal pancreatic cells harbor low levels of EGFR while the upregulation of EGFR is already detectable at early 
stages of pancreatic carcinogenesis (Park et al. 2015). Overexpression of the EGF receptor has been associated with poor prognosis in many human cancer types. In breast cancer, high level of EGFR is correlated with short relapse-free interval and survival (Foley et al. 2010). And in human gliomas, patients baring overexpressed EGFR are with higher risk of aggressiveness (Doherty et al. 2006). In pancreatic cancer, the significance of endogenous EGFR activity was, for a long time dismissed, as its downstream target KRAS, when mutated, stays in a constitutive active state, which theoretically, should no longer need upstream stimulation. However, it is noticed that the concomitant activation of Kras and TGFa overexpression greatly accelerates the progression from ADM to PDAC, suggesting the functional non-redundancy of Kras ${ }^{G 12 D}$ and EGFR signaling (Siveke et al. 2007).

Studies using $\operatorname{Kras}^{G 12 D}$ transgenic mouse models have identified a cooperative manner between EGFR signaling activity and oncogenic Kras in promoting pancreatic carcinogenesis. EGFR pathway upregulation and activation is found in $K r a s^{G 12 D}$ mice prior to pre-neoplastic lesion formation. When blocking EGFR signaling activity by depleting Egfr, neoplastic lesion formation is almost completely prohibited, suggesting that EGFR activity is instrumental in complementing $\mathrm{Kras}^{\mathrm{G12D}}$-driven metaplastic processes (Ardito et al. 2012; Siveke et al. 2012). In our study, a similar implemental role of EGFR signaling activation in accelerating ADM formation is seen in the context of Arid1a deficiency. EGFR activation by ligand stimulation is known to induce ADM formation in vitro (Means et al. 2005). Strikingly, this effect can be drastically boosted when EGFR activation occurs in with the context of Arid1a deficiency. To better understand this cooperative manner, we sought to examine the EGFR expression level in mice pancreas lacking ARID1A. Total EGFR is significantly upregulated in 8-week-old Arid1a deficient pancreas, especially gathered in the metaplastic lesion areas. Pathway analyses of the genes that gain accessibility upon Arid1a loss also prompted the involvement of EGFR activity by showing high enrichment of EGF-inducible genes. The cooperative pattern of EGFR activation and Arid1a deficiency is not restricted to the Kras-wildtype condition, but also applied to the acinar cells with Kras ${ }^{G 12 D}$ mutations. In 266-6 cells, the most prominent ADM yield is seen when combining EGF treatment and Arid1a knockdown, just not as efficient as in primary acinar cells, which most probably is due to the limited period of validity of siRNA transfection.

The transdifferentiation from cells with acinar feature to ductal-like cells is controlled at 
the level of gene transcription. Both EGF treatment and Arid1a knockdown cause a cellular transcriptional shift from genes that characterize acinar identity, such as acinar differentiation maintaining genes Ptf1a, Bhlha15 and Nr5a2, as well as genes encoding trypsinogen (Prss1 and Prss3), to ductal genes which encode keratin (Krt8, Krt18 and Krt20), E-Cadherin (Cdh1) and matricellular protein osterpontin (Spp1) (Kilic et al. 2006). And notably, EGFR signaling cooperates with Arid1a deficiency in facilitating ductal genes transcription, rather than restraining expression of acinar markers, suggesting that the activation of ductal-gene expression profiles as a prerequisite for the ADM boosted by combined EGFR activation and Arid1a loss. Pathway analysis of those genes which are upregulated upon sole EGF treatment, sole Arid1a knockdown and combination of these two conditions link those cooperatively regulated genes to organ regeneration and epithelial cell differentiation, thus highlighting the role of Arid1a deficiency and EGFR signaling in modulating acinar organization and reprogramming.

The universality of the cooperation between EGFR activity and Arid1a deficiency, regardless of the Kras status, suggests that the downstream pathway which relays signals for ADM initiation might be common for mutant and wild-type Kras. Major EGFR downstream signaling hubs that are shared by wild-type and mutant Kras are PI3K and serine/threonine-protein kinase D1 (PRKD1) (Storz 2017). Studies showed that EGFRdependent Mek/Erk activation is required for tumor initiation in vivo and ADM in vitro, suggesting that cooperation of EGFR activation and Arid1a deficiency might function via Mek/Erk in promoting ADM (Siveke et al. 2012; Ardito et al. 2012). As for the mechanism which leads to EGFR upregulation in Kras-wildtype Arid1a-deficient acinar cells, we propose the involvement of NF-KB signaling. NF-KB signaling is downstream of PRKD1 and has been linked to prominent oncogenic activity in PDAC. Its activation induces upregulation of EGFR signaling components (EGFR/EGF/TGF $\alpha$ ), which further supports the dedifferentiation of acinar cells (Liou et al. 2016). In our RNA-seq analysis, activation of NF-KB is associated with Arid1a deficient phenotype where we also detect EGFR upregulation, implying the contribution of this signaling pathway in facilitating EGFR overexpression.

\subsubsection{The role of EGFR signaling in regulating genomic disposition of ARID1A}

Given that ARID1A-mediated chromatin remodeling plays a fundamental role in transcription regulation of key cellular processes including acinar reprogramming, we 
hypothesized that the enhanced acinar cell transdifferentiation led by EGFR signaling activation and Arid1a deficiency may result from altered chromatin modulation. To this end, we checked the global genomic occupancy of ARID1A following EGFR treatment. Unexpectedly, EGF stimulation resulted in a global decrease of ARID1A occupancy, although total ARID1A expression remained unchanged. Consistently, immunoblot analysis exhibits a release of ARID1A from chromatin-enriched fraction to nucleoplasm upon EGF treatment. However, it remains unknown if this allocation shift confers ARID1A any novel biological function besides releasing control from the previous targets.

We have shown that EGFR signaling activity and Arid1a deficiency cooperatively facilitate ADM and ductal gene expression. To decipher the direct role of ARID1A in this process, we examined ARID1A occupancy on the 213 genes displaying cooperative upregulation by joint EGF stimulation and Arid1a loss. We found that ARID1A directly binds $81(38 \%)$ of these genes and its occupancy decreases from a majority (83\%) of these sites in response to EGFR signaling activation. The genes that are released from the control of ARID1A upon EGF stimulation are involved in the processes of EMT and development regulation, as well as with an unfavorable prognosis of pancreatic cancer. One of these target genes, Bhlhe40 encodes for a basic helix-loop-helix transcription factor that participates in regulating cell proliferation and apoptosis (Wu et al. 2012). High expression of BHLHE40 has been linked to a pro-metastatic phenotype in various cancers, including hepatocellular carcinoma, breast cancer and pancreatic cancer (Xiong et al. 2016; Chakrabarti et al. 2004; Wu et al. 2012). Wu et al. demonstrated that BHLHE40 promotes EMT in response to TGF $\beta$ signaling in PDAC cells. Concomitant with Bhlhe40 upregulation, we also found that Snai1, another EMT-related factor, is cooperatively upregulated by EGF stimulation and Arid1a-knockdown, again suggesting the high transdifferentiation activity of acinar cells under the combined treatment. Recently, Lif (leukemia inhibitory factor) was shown to be upregulated in oncogenic Krasdriven pancreatic cancer and blockade of LIF activity by neutralizing antibody suppresses pancreatic malignancy (Wang et al. 2019). MET, encoded by c-Met oncogene, is a multifunctional cytokine which mediates morphogenesis and invasive growth of epithelial cells (Di Renzo et al. 1995; Delitto et al. 2014). These genes may constitute an integral transcription program that directly or indirectly promote acinar-toductal conversion. 


\subsubsection{EGFR signaling leads to genomic ARID1A dissociation via activating NFATc1}

The nuclear factor of activated T cells (NFAT) family of transcription factors, as primarily discovered in T cells, are most extensively studied and best characterized as regulating development and differentiation processes in the immune system. In the canonical pathway, NFAT is activated by $\mathrm{Ca}^{2+}$ flux and the subsequently activated calmodulincalcineurin cascade to translocate into the nucleus to initiate transcription of target genes (Macian et al. 2005; Rao et al. 1997; Chen et al. 1998). This pathway can be blocked by cyclosporin A (CsA)-mediated calcineurin inhibition (Mancini et al. 2009). Accumulative evidences show that NFAT expression is not restricted to the immune system, instead, they are ubiquitously expressed in almost all human tissues, and a role for these transcription factors in carcinogenesis is also emerging (Mancini et al. 2009; Chang et al. 2004; Mammucari et al. 2005; Jauliac et al. 2002). In pancreatic cancer, NFAT, especially the family member NFATc1 acts as a crucial factor that integrates inflammatory signals into malignant transformation. In particular, NFATc1 is strongly induced upon inflammatory stimuli and subsequently cooperates with STAT3 or AP-1 to accelerate Kras $^{G 12 D}$-driven carcinogenesis (Buchholz et al. 2006; Baumgart et al. 2014). Further, NFATc1 is directly incorporated into EGFR signaling, based on the finding that ectopic NFATc1 expression in pancreatic cancer tissue is a result of EGFR activation (Chen et al. 2015). Notably, NFATc1 silencing by pharmacological or genetic approaches significantly decreases EGFR-mediated ductal gene expression and blocks acinar cell transdifferentiation, both in vitro and in vivo, highlighting the significance of EGFRNFATc1 axis in regulating acinar plasticity (Chen et al. 2015). In our study, EGFR signaling dissociates ARID1A from duct-promoting genes and leads to a similar metaplastic phenotype as NFATc1 overexpression, therefore, we highly suspect that NFATc1 is correlated to EGFR-ARID1A regulation. This hypothesis is supported by the finding that the consensus site of NFAT is greatly enriched in ARID1A-bound genomic regions and NFATc1 does bind a subset of ARID1A target genes, as is shown by ChIPqPCR. Besides, genes that regulate cellular plasticity, as defined with ARID1A occupancy as well as EGFR- and Arid1a-knockdown induced activation, show a considerable overlay with NFATc1-inducible genes. Importantly, mice with pancreasspecific expression of active NFATc1 exhibited similar phenotype as Arid1a-deficient mice that they both displayed ADM and developed pancreatic atrophy and loss of exocrine mass (Chen et al. 2017). Consistent with the previous study, NFATc1 
transcriptional activity is enhanced by EGFR signaling activation (Chen et al. 2015), interestingly however, Arid1a-knockdown has a comparable effect in eliciting NFATc1 activity. Consistently, blocking the nuclear translocation of NFATc1 using CsA, the induction of the target genes led by EGF treatment and Arid1a depletion is significantly abrogated. Together with the impairment of the transcriptional activation of ductal genes, acinar to ductal conversion is also eliminated by NFATc1 activity blockage. In all, our findings demonstrate a role for NFATc1 as a pivotal hub in ADM-related transcription regulation led by EGFR signaling and Arid1a depletion.

Since EGFR-dependent NFATc1 induction also causes ADM in cells without genetic Arid1a ablation (Chen et al. 2015; our study), we asked whether ARID1A genomic dissociation is a consequence of EGFR-induced NFATc1 activation. By intersecting genes which gain expression upon cooperative regulation of EGFR signaling and Arid1a deficiency with NFATc1 targets, we find that NFATc1 preferentially binds genomic sites that lose ARID1A upon EGF treatment. Moreover, manipulating NFATc1 expression by overexpressing constitutive active NFATc1 results in a significant decrease of the chromatin-enriched ARID1A and in an increase of the nucleoplasmic ARID1A fraction. Consistently, CsA-mediated NFATc1 inhibition accumulates ARID1A on chromatin and rescues ARID1A occupancy on its target genes. Therefore, we conclude that EGFRinduced NFATc1 nuclear translocation is sufficient to disassociate ARID1A from the genome, thus intervening with the ARID1A-dependent transcription regulation to promote acinar reprogramming (Figure 26). 


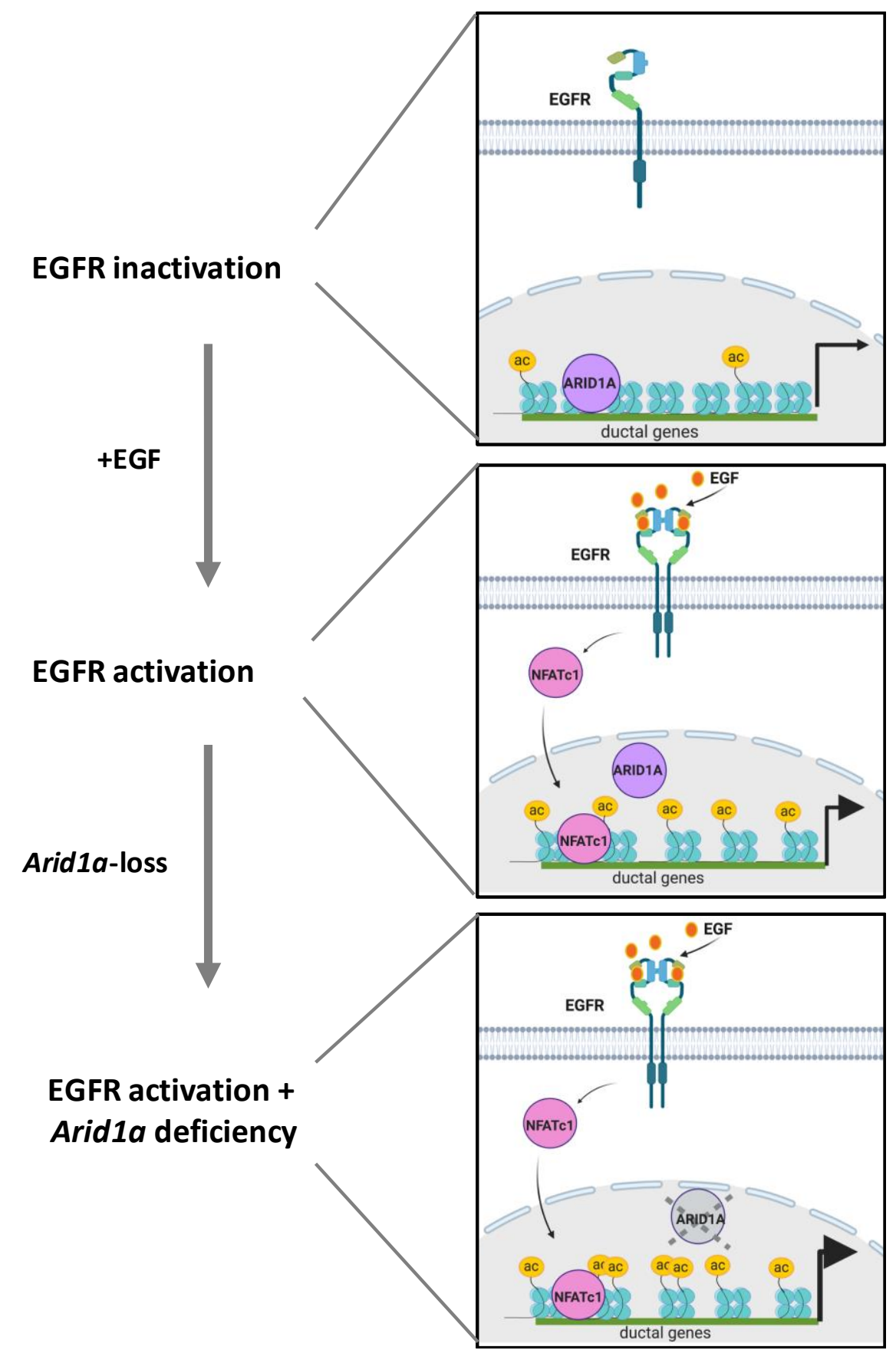

Figure 26. Model of EGFR-ARID1A-axis induced metaplastic transcriptional activation. ARID1A binds and prevents ductal genes from hyper-acetylation. Upon ligand-stimulated EGFR signaling activation, NFATc1 transcription factor translocates into the nucleus and dissociates ARID1A from the genome followed by activation of ductal gene signatures. 


\subsection{Concluding remarks}

Dysregulated chromatin remodeling caused by mutations of SWI/SNF subunits has emerged as a mechanism leading to pancreatic pathogenesis. Several studies demonstrated the role of ARID1A in maintaining pancreatic homeostasis and preventing carcinogenesis, however, the precise mechanism of how ARID1A mediates these processes with its intrinsic chromatin remodeling capability remains unclear, so does the functional relevance of ARID1A in the context of other oncogenic signaling pathways. By combining a functional analysis in a transgenic mouse model of Arid1a deficiency and a murine acinar cell line with transcriptome and chromatin analysis, we dissect the functional implications and interacting signaling cascades of Arid1a deficiency in modulating acinar cell reprogramming.

The main findings of the study are:

- $\quad$ ARID1A is necessary for maintaining pancreatic acinar cell integrity, with loss of ARID1A resulting in acinar-to-ductal conversion and formation of preneoplastic pancreatic lesions.

- Arid1a deficiency increases chromatin accessibility and allows transcriptional activation of metaplastic/ductal gene programs.

- ARID1A directly binds the enhancer elements of genes that regulate pancreas development and differentiation.

- $\quad$ Arid1a deficiency and EGFR signaling-induced NFATc1 activation cooperate in promoting ADM-permissive transcription programs

- EGFR signaling phenocopies Arid1a deficiency in driving acinar reprogramming by reducing genome-wide ARID1A occupancy.

- $\quad$ EGFR-induced NFATc1 activation elicits genomic ARID1A dissociation and drives metaplastic gene programs required for the ADM phenotype.

Taken together, we elucidate an integral regulation loop of chromatin remodeling, environmental stimuli and transcription factor activity in mediating acinar cell plasticity. 


\section{References}

Ahern M J, Harrison W, Hollingsworth $\mathrm{P}$, et al. A randomised double-blind trial of cyclosporin and azathioprine in refractory rheumatoid arthritis[J]. Australian and New Zealand journal of medicine, 1991, 21(6): 844-849.

Aichler $M$, Seiler $C$, Tost $M$, et al. Origin of pancreatic ductal adenocarcinoma from atypical flat lesions: a comparative study in transgenic mice and human tissues[J]. The Journal of pathology, 2012, 226(5): 723-734.

Akagi H, Reynolds A, Hjelm M. Cyclosporin A and its metabolites, distribution in blood and tissues[J]. Journal of international medical research, 1991, 19(1): 1-18.

Alexander J M, Hota S K, He D, et al. Brg1 modulates enhancer activation in mesoderm lineage commitment[J]. Development, 2015, 142(8): 1418-1430.

Allo G, Bernardini M Q, Wu R C, et al. ARID1A loss correlates with mismatch repair deficiency and intact p53 expression in high-grade endometrial carcinomas[J]. Modern Pathology, 2014, 27(2): 255-261.

Alver, Burak H., et al. "The SWI/SNF chromatin remodelling complex is required for maintenance of lineage specific enhancers." Nature communications 8.1 (2017): 110.

Arda H E, Benitez C M, Kim S K. Gene regulatory networks governing pancreas development[J]. Developmental cell, 2013, 25(1): 5-13.

Ardito C M, Grüner B M, Takeuchi K K, et al. EGF receptor is required for KRAS-induced pancreatic tumorigenesis[J]. Cancer cell, 2012, 22(3): 304-317.

Asanoma K, Hori E, Yoshida S, et al. Mutual suppression between BHLHE40/BHLHE41 and the MIR301B-MIR130B cluster is involved in epithelialto-mesenchymal transition of endometrial cancer cells[J]. Oncotarget, 2019, 10(45): 4640.

Attanasio C, Nord A S, Zhu Y, et al. Tissue-specific SMARCA4 binding at active and repressed regulatory elements during embryogenesis[J]. Genome research, 2014, 24(6): 920-929. 
Bagchi, Anindya, and Alea A. Mills. "The quest for the 1p36 tumor suppressor." Cancer research 68.8 (2008): 2551-2556.

Baumann V, Wiesbeck M, Breunig C T, et al. Targeted removal of epigenetic barriers during transcriptional reprogramming[J]. Nature communications, 2019, 10(1): 112.

Baumgart S, Chen N M, Siveke J T, et al. Inflammation-Induced NFATc1-STAT3 transcription complex promotes pancreatic cancer initiation by KrasG12D[J]. Cancer discovery, 2014, 4(6): 688-701.

Beals C R, Clipstone N A, Ho S N, et al. Nuclear localization of NF-ATc by a calcineurin-dependent, cyclosporin-sensitive intramolecular interaction[J]. Genes \& development, 1997, 11(7): 824-834.

Belkina A C, Nikolajczyk B S, Denis G V. BET protein function is required for inflammation: Brd2 genetic disruption and BET inhibitor JQ1 impair mouse macrophage inflammatory responses[J]. The Journal of Immunology, 2013, 190(7): 3670-3678.

Biddie S C, John S, Sabo P J, et al. Transcription factor AP1 potentiates chromatin accessibility and glucocorticoid receptor binding[J]. Molecular cell, 2011, 43(1): 145-155.

Biegel J A, Fogelgren B, Zhou J Y, et al. Mutations of the INI1 rhabdoid tumor suppressor gene in medulloblastomas and primitive neuroectodermal tumors of the central nervous system[J]. Clinical cancer research, 2000, 6(7): 2759-2763.

Birchmeier C, Birchmeier W, Gherardi E, et al. Met, metastasis, motility and more[J]. Nature reviews Molecular cell biology, 2003, 4(12): 915-925.

Boj S F, Hwang C I, Baker LA, et al. Organoid models of human and mouse ductal pancreatic cancer[J]. Cell, 2015, 160(1-2): 324-338.

Bossen C, Murre C S, Chang A N, et al. The chromatin remodeler Brg1 activates enhancer repertoires to establish $B$ cell identity and modulate cell growth[J]. Nature immunology, 2015, 16(7): 775-784.

Breeden L, Nasmyth K (1987) Cell cycle control of the yeast HO gene: cis- and transacting regulators. Cell 48(3):389 -397. 
Buchholz M, Schatz A, Wagner M, et al. Overexpression of c-myc in pancreatic cancer caused by ectopic activation of NFATc1 and the Ca2+/calcineurin signaling pathway[J]. The EMBO journal, 2006, 25(15): 3714-3724.

Burns L G, Peterson C L. The yeast SWI-SNF complex facilitates binding of a transcriptional activator to nucleosomal sites in vivo[J]. Molecular and cellular biology, 1997, 17(8): 4811-4819.

Burris, HA 3rd, et al. "Improvements in survival and clinical benefit with gemcitabine as first-line therapy for patients with advanced pancreas cancer: a randomized trial." Journal of clinical oncology 15.6 (1997): 2403-2413.

Cooper CL, O'Toole SA, Kench JG. Classification, morphology and molecular pathology of premalignant lesions of the pancreas. Pathology 2013; 45: 286-304 [PMID: 23442735 DOI: 10.1097/PAT. 0b013e32835f2205]

Caumanns, Joseph J., et al. "ARID1A mutant ovarian clear cell carcinoma: A clear target for synthetic lethal strategies." Biochimica et Biophysica Acta (BBA)Reviews on Cancer 1870.2 (2018): 176-184.

Centore R C, Sandoval G J, Soares L M M, et al. Mammalian SWI/SNF chromatin remodeling complexes: emerging mechanisms and therapeutic strategies[J]. Trends in Genetics, 2020.

Chakrabarti J, Turley H, Campo L, et al. The transcription factor DEC1 (stra13, SHARP2) is associated with the hypoxic response and high tumour grade in human breast cancers[J]. British journal of cancer, 2004, 91(5): 954-958.

Chandler R L, Brennan J, Schisler J C, et al. ARID1a-DNA interactions are required for promoter occupancy by SWI/SNF[J]. Molecular and cellular biology, 2013, 33(2): 265-280.

Chang, C. P., Neilson, J. R., Bayle, J. H., Gestwicki, J. E., Kuo, A., Stankunas, K., ... \& Crabtree, G. R. (2004). A field of myocardial-endocardial NFAT signaling underlies heart valve morphogenesis. Cell, 118(5), 649-663.

Chen L, Glover J N M, Hogan P G, et al. Structure of the DNA-binding domains from NFAT, Fos and Jun bound specifically to DNA[J]. Nature, 1998, 392(6671): 42-48. 
Chen N M, Singh G, Koenig A, et al. NFATc1 links EGFR signaling to induction of Sox9 transcription and acinar-ductal transdifferentiation in the pancreas[J].

Gastroenterology, 2015, 148(5): 1024-1034. e9.

Cherry A B C, Daley G Q. Reprogramming cellular identity for regenerative medicine[J]. Cell, 2012, 148(6): 1110-1122.

Chi T H, Wan M, Zhao K, et al. Reciprocal regulation of CD4/CD8 expression by SWI/SNF-like BAF complexes[J]. Nature, 2002, 418(6894): 195-199.

Choo M K, Yeo H, Zayzafoon M. NFATc1 mediates HDAC-dependent transcriptional repression of osteocalcin expression during osteoblast differentiation[J]. Bone, 2009, 45(3): 579-589.

Citri A, Yarden Y. EGF-ERBB signalling: towards the systems level. Nat Rev Mol Cell Biol 2006;7:505-16

Clapier CR, Cairns BR. The biology of chromatin remodeling complexes. Annu Rev Biochem 2009;78:273-304. [PubMed: 19355820]

Collisson E A, Sadanandam A, Olson P, et al. Subtypes of pancreatic ductal adenocarcinoma and their differing responses to therapy[J]. Nature medicine, 2011, 17(4): 500-503.

Cosma M P. Ordered recruitment: gene-specific mechanism of transcription activation[J]. Molecular cell, 2002, 10(2): 227-236.

Crabtree G R. Generic signals and specific outcomes: signaling through $\mathrm{Ca} 2+$, calcineurin, and NF-AT[J]. Cell, 1999, 96(5): 611-614.

Cristofaro M F D, Betz B L, Rorie C J, et al. Characterization of SWI/SNF protein expression in human breast cancer cell lines and other malignancies[J]. Journal of cellular physiology, 2001, 186(1): 136-145.

Dallas, P. B., Pacchione, S., Wilsker, D., Bowrin, V., Kobayashi, R., \& Moran, E. (2000). The human SWI-SNF complex protein p270 is an ARID family member with nonsequence-specific DNA binding activity. Molecular and cellular biology, 20(9), 31373146.

Dawson JP, Berger MB, Lin CC, Schlessinger J, Lemmon MA, Ferguson KM: Epidermal 
growth factor receptor dimerization and activation require ligand-induced conformational changes in the dimer interface. Mol Cell Biol 2005;25:7734-7742.

De R C L, Logsdon C D. Pancreatic acinar cells in culture: expression of acinar and ductal antigens in a growth-related manner[J]. European journal of cell biology, 1990, 51(1): 64-75.

Delitto D, Vertes-George E, Hughes S J, et al. c-Met signaling in the development of tumorigenesis and chemoresistance: potential applications in pancreatic cancer[J]. World journal of gastroenterology: WJG, 2014, 20(26): 8458.

Delmore J E, Issa G C, Lemieux M E, et al. BET bromodomain inhibition as a therapeutic strategy to target c-Myc[J]. Cell, 2011, 146(6): 904-917.

Delpu Y, Hanoun N, Lulka H, Sicard F, Selves J, Buscail L, Torrisani J, Cordelier P. Genetic and epigenetic alterations in pa- ncreatic carcinogenesis. Curr Genomics 2011; 12: 15-24 [PMID: 21886451 DOI: 10.2174/138920211794520132]

Di Marco, Mariacristina, et al. "Metastatic pancreatic cancer: is gemcitabine still the best standard treatment?." Oncology reports 23.5 (2010): 1183-1192.

Di Renzo M F, Poulsom R, Olivero M, et al. Expression of the Met/hepatocyte growth factor receptor in human pancreatic cancer[J]. Cancer research, 1995, 55(5): 11291138.

Doherty L, Gigas D C, Kesari S, et al. Pilot study of the combination of EGFR and mTOR inhibitors in recurrent malignant gliomas[J]. Neurology, 2006, 67(1): 156-158.

Dong P D S, Provost E, Leach S D, et al. Graded levels of Ptf1a differentially regulate endocrine and exocrine fates in the developing pancreas[J]. Genes \& development, 2008, 22(11): 1445-1450.

Edlund, H. Pancreatic organogenesis - developmental mechanisms and implications for therapy. Nat Rev Genet 3, 524-532 (2002). https://doi.org/10.1038/nrg841

Eiriksdottir G, Sigurdsson A, Jonasson J G, et al. Loss of heterozygosity on chromosome 9 in human breast cancer: association with clinical variables and genetic changes at other chromosome regions[J]. International journal of cancer, 1995, 64(6): 378382. 
Feldmann G, Beaty R, Hruban RH, Maitra A. Molecular genetics of pancreatic intraepithelial neoplasia. J Hepatobiliary Pancreat Surg. 2007;14(3):224-32. doi: 10.1007/s00534-006-1166-5. Epub 2007 May 29. PMID: 17520196; PMCID: PMC2666331.

Ferri-Borgogno S, Barui S, McGee A M, et al. Paradoxical role of AT-rich interactive domain $1 \mathrm{~A}$ in restraining pancreatic carcinogenesis[J]. bioRxiv, 2019: 835793.

Filippakopoulos P, Qi J, Picaud S, et al. Selective inhibition of BET bromodomains[J]. Nature, 2010, 468(7327): 1067-1073.

Flores-Alcantar A, Gonzalez-Sandoval A, Escalante-Alcalde D, et al. Dynamics of expression of ARID1A and ARID1B subunits in mouse embryos and in cells during the cell cycle[J]. Cell and tissue research, 2011, 345(1): 137-148.

Foley J, Nickerson N K, Nam S, et al. EGFR signaling in breast cancer: bad to the bone[C]//Seminars in cell \& developmental biology. Academic Press, 2010, 21(9): 951-960.

Fryer C J, Archer T K. Chromatin remodelling by the glucocorticoid receptor requires the BRG1 complex[J]. Nature, 1998, 393(6680): 88-91.

Gao X, Tate P, Hu P, et al. ES cell pluripotency and germ-layer formation require the SWI/SNF chromatin remodeling component BAF250a[J]. Proceedings of the National Academy of Sciences, 2008, 105(18): 6656-6661.

Gao, F., Elliott, N. J., Ho, J., Sharp, A., Shokhirev, M. N., \& Hargreaves, D. C. (2019). Heterozygous mutations in SMARCA2 reprogram the enhancer landscape by global retargeting of SMARCA4. Molecular cell, 75(5), 891-904.

Germond JE, Hirt P, Oudet P, Gross-Bellard M, Chambon P . Proc. Natl. Acad Sci. USA 1975, 72: 1843-1847

Gillen S, Schuster T, Meyer Zum Büschenfelde C, et al. Preoperative/neoadjuvant therapy in pancreatic cancer: a systematic review and meta-analysis of response and resection percentages. PLoS Med 2010; 7: e1000267.

Giri A K, Parekatt V, Dwivedi O P, et al. Common variants of ARID1A and KAT2B are associated with obesity in Indian adolescents[J]. Scientific reports, 2018, 8(1): 1-7. 
Glaros S, Cirrincione G M, Muchardt C, et al. The reversible epigenetic silencing of BRM: implications for clinical targeted therapy[J]. Oncogene, 2007, 26(49): 7058-7066.

Görisch S M, Wachsmuth M, Tóth K F, et al. Histone acetylation increases chromatin accessibility[J]. Journal of cell science, 2005, 118(24): 5825-5834.

Graef, Isabella A., Feng Chen, and Gerald R. Crabtree. "NFAT signaling in vertebrate development." Current opinion in genetics \& development 11.5 (2001): 505-512.

Graf T. Historical origins of transdifferentiation and reprogramming[J]. Cell stem cell, 2011, 9(6): 504-516.

Grapin-Botton A. Ductal cells of the pancreas[J]. The international journal of biochemistry \& cell biology, 2005, 37(3): 504-510.

Guan, Bin, Tian-Li Wang, and le-Ming Shih. "ARID1A, a factor that promotes formation of SWI/SNF-mediated chromatin remodeling, is a tumor suppressor in gynecologic cancers." Cancer research 71.21 (2011): 6718-6727.

Hasselluhn M C, Schmidt G E, Ellenrieder V, et al. Aberrant NFATc1 signaling counteracts TGF $\beta$-mediated growth arrest and apoptosis induction in pancreatic cancer progression[J]. Cell death \& disease, 2019, 10(6): 1-14.

Haynes S R, Dollard C, Winston F, et al. The bromodomain: a conserved sequence found in human, Drosophila and yeast proteins[J]. Nucleic acids research, 1992, 20(10): 2603.

Heery, David M., et al. "A signature motif in transcriptional co-activators mediates binding to nuclear receptors." Nature 387.6634 (1997): 733-736

Helming, K.C.; Wang, X.; Wilson, B.G.; Vazquez, F.; Haswell, J.R.; Manchester, H.E.; Kim, Y.; Kryukov, G.V.; Ghandi, M.; Aguirre, A.J.; et al. ARID1B is a specific vulnerability in ARID1A-mutant cancers. Nat. Med. 2014, 20, 251-254.

Hemming AW, Davis NL, Kluftinger A, Robinson B, Quenville NF, Liseman B, LeRiche J: Prognostic markers of colorectal cancer: an evaluation of DNA content, epidermal growth factor receptor, and Ki-67. J Surg Oncol 1992;51:147-152.

Hill D A, Chiosea S, Jamaluddin S, et al. Inducible changes in cell size and attachment area due to expression of a mutant SWI/SNF chromatin remodeling enzyme[J]. 
Journal of cell science, 2004, 117(24): 5847-5854.

Hingorani S R, Petricoin III E F, Maitra A, et al. Preinvasive and invasive ductal pancreatic cancer and its early detection in the mouse[J]. Cancer cell, 2003, 4(6): 437-450.

Hoffman G R, Rahal R, Buxton F, et al. Functional epigenetics approach identifies BRM/SMARCA2 as a critical synthetic lethal target in BRG1-deficient cancers[J]. Proceedings of the National Academy of Sciences, 2014, 111(8): 3128-3133.

Hogan P G, Chen L, Nardone J, et al. Transcriptional regulation by calcium, calcineurin, and NFAT[J]. Genes \& development, 2003, 17(18): 2205-2232.

Hota S K, Bruneau B G. ATP-dependent chromatin remodeling during mammalian development[J]. Development, 2016, 143(16): 2882-2897.

Hruban RH, Adsay NV, Albores-Saavedra J, Compton C, Garrett ES, Goodman SN, Kern SE, Klimstra DS, Klöppel G, Longnecker DS, Lüttges J, Offerhaus GJ. Pancreatic intraepithelial neoplasia: a new nomenclature and classification system for pancreatic duct lesions. Am J Surg Pathol 2001; 25: 579-586 [PMID: 11342768 DOI: 10.1097/00000478-200105000-00003]

Hu G, Schones D E, Cui K, et al. Regulation of nucleosome landscape and transcription factor targeting at tissue-specific enhancers by BRG1[J]. Genome research, 2011, 21(10): 1650-1658.

Huang, Zhi-Qing, Jiwen Li, Laurent M. Sachs, Philip A. Cole, and Jiemin Wong. "A role for cofactor-cofactor and cofactor-histone interactions in targeting p300, SWI/SNF and Mediator for transcription." The EMBO Journal 22, no. 9 (2003): 2146-2155.

Hynes NE, Lane HA. ErbB receptors and cancer: the complexity of targeted inhibitors. Nat Rev Cancer 2005; 5: 341.

Isakoff M S, Sansam C G, Tamayo P, et al. Inactivation of the Snf5 tumor suppressor stimulates cell cycle progression and cooperates with p53 loss in oncogenic transformation[J]. Proceedings of the National Academy of Sciences, 2005, 102(49): $17745-17750$.

Ito T, Yamauchi M, Nishina M, et al. Identification of SWI· SNF complex subunit BAF60a as a determinant of the transactivation potential of Fos/Jun dimers[J]. Journal of Biological Chemistry, 2001, 276(4): 2852-2857. 
Ivana L, Ohkawa Y, Imbalzano A N. Chromatin remodelling in mammalian differentiation: lessons from ATP-dependent remodellers[J]. Nature Reviews Genetics, 2006, 7(6): 461-473.

J.M.Slack. Developmental biology of the pancreas. Development 1995, 121: 1569-1580. Jain J, McCaffrey P G, Valge-Archer V E, et al. Nuclear factor of activated T cells contains Fos and Jun[J]. Nature, 1992, 356(6372): 801-804.

Jauliac S, López-Rodriguez C, Shaw L M, et al. The role of NFAT transcription factors in integrin-mediated carcinoma invasion[J]. Nature cell biology, 2002, 4(7): 540-544.

Jeanmougin F, Wurtz J M, Le Douarin B, et al. The bromodomain revisited[J]. Trends in biochemical sciences, 1997, 22(5): 151-153.

Jeon $\mathrm{H} \mathrm{M}$, Lee J. MET: roles in epithelial-mesenchymal transition and cancer stemness[J]. Annals of translational medicine, 2017, 5(1).

Ji B, Tsou L, Wang $\mathrm{H}$, et al. Ras activity levels control the development of pancreatic diseases[J]. Gastroenterology, 2009, 137(3): 1072-1082. e6.

Jimeno A, Tan A C, Coffa J, et al. Coordinated epidermal growth factor receptor pathway gene overexpression predicts epidermal growth factor receptor inhibitor sensitivity in pancreatic cancer[J]. Cancer research, 2008, 68(8): 2841-2849.

Johnson C L, Peat J M, Volante $S \mathrm{~N}$, et al. Activation of protein kinase C $\delta$ leads to increased pancreatic acinar cell dedifferentiation in the absence of MIST1[J]. The Journal of pathology, 2012, 228(3): 351-365.

Jones S, Wang T L, Shih I M, et al. Frequent mutations of chromatin remodeling gene ARID1A in ovarian clear cell carcinoma[J]. Science, 2010, 330(6001): 228-231.

Kadoch C, Hargreaves D C, Hodges C, et al. Proteomic and bioinformatic analysis of mammalian SWI/SNF complexes identifies extensive roles in human malignancy[J]. Nature genetics, 2013, 45(6): 592-601.

Kelso T W R, Porter D K, Amaral M L, et al. Chromatin accessibility underlies synthetic lethality of SWI/SNF subunits in ARID1A-mutant cancers[J]. Elife, 2017, 6: e30506.

Kilic G, Wang J, Sosa-Pineda B. Osteopontin is a novel marker of pancreatic ductal tissues and of undifferentiated pancreatic precursors in mice[J]. Developmental 
dynamics: an official publication of the American Association of Anatomists, 2006, 235(6): 1659-1667.

Kim M, Lu F, Zhang Y. Loss of HDAC-mediated repression and gain of NF-KB activation underlie cytokine induction in ARID1A-and PIK3CA-mutation-driven ovarian cancer[J]. Cell reports, 2016, 17(1): 275-288.

Kim T K, Hemberg M, Gray J M. Enhancer RNAs: a class of long noncoding RNAs synthesized at enhancers[J]. Cold Spring Harbor perspectives in biology, 2015, 7(1): a018622.

Kimura Y, Fukuda A, Ogawa S, et al. ARID1A maintains differentiation of pancreatic ductal cells and inhibits development of pancreatic ductal adenocarcinoma in mice[J]. Gastroenterology, 2018, 155(1): 194-209. e2.

Klein P, Mattoon D, Lemmon MA, Schlessinger J: A structure-based model for ligand binding and dimerization of EGF receptors. Proc Natl Acad Sci USA 2004;101:929_ 934.

Klimstra DS, Longnecker DS. K-ras mutations in pancreatic ductal proliferative lesions. Am J Pathol 1994; 145: 1547-1550 [PMID: 7992857]

Klochendler-Yeivin A, Fiette L, Barra J, Muchardt C, Babinet C, Yaniv M. The murine SNF5/INI1 chromatin remodeling factor is essential for embryonic development and tumor suppression. EMBO Rep 2000;1:500-6

Klochendler-Yeivin A, Picarsky E, Yaniv M. Increased DNA damage sensitivity and apoptosis in cells lacking the Snf5/Ini1 subunit of the SWI/SNF chromatin remodeling complex[J]. Molecular and cellular biology, 2006, 26(7): 2661-2674.

Köenig A, Linhart T, Schlengemann K, et al. NFAT-induced histone acetylation relay switch promotes c-Myc-dependent growth in pancreatic cancer cells[J]. Gastroenterology, 2010, 138(3): 1189-1199. e2.

Kopp J L, von Figura G, Mayes E, et al. Identification of Sox9-dependent acinar-to-ductal reprogramming as the principal mechanism for initiation of pancreatic ductal adenocarcinoma[J]. Cancer cell, 2012, 22(6): 737-750.

Korc M, Friess $\mathrm{H}$, Yamanaka $\mathrm{Y}$, et al. Chronic pancreatitis is associated with increased concentrations of epidermal growth factor receptor, transforming growth factor alpha, Page | 98 
and phospholipase C gamma[J]. Gut, 1994, 35(10): 1468-1473.

Kornberg RD. Chromatin structure: a repeating unit of histones and DNA. Science 1974;184:868-71. [PubMed: 4825889]

Kowenz-Leutz E, Leutz A. AC/EBP $\beta$ isoform recruits the SWI/SNF complex to activate myeloid genes[J]. Molecular cell, 1999, 4(5): 735-743.

Langer L F, Ward J M, Archer T K. Tumor suppressor SMARCB1 suppresses superenhancers to govern hESC lineage determination[J]. Elife, 2019, 8: e45672.

Lasko L M, Jakob C G, Edalji R P, et al. Discovery of a selective catalytic p300/CBP inhibitor that targets lineage-specific tumours[J]. Nature, 2017, 550(7674): 128-132.

Lee J, Snyder E R, Liu Y, et al. Reconstituting development of pancreatic intraepithelial neoplasia from primary human pancreas duct cells[J]. Nature communications, 2017, 8(1): 1-14.

Lee S, Heinrich E L, Lu J, et al. Epidermal growth factor receptor signaling to the MAPK pathway bypasses RAS in pancreatic cancer cells[J]. Pancreas, 2016, 45(2): 286.

Lei i, Gao X, Sham MH, et al. SWi/SnF protein component BaF250a regulates cardiac progenitor cell differentiation by modulating chromatin accessibility during second heart field development. J Biol Chem 2012;287:24255-62.

Levine M, Tjian R. Transcription regulation and animal diversity[J]. Nature, 2003, 424(6945): 147-151.

Levy C S, Slomiansky V, Gattelli A, et al. Tumor necrosis factor alpha induces LIF expression through ERK1/2 activation in mammary epithelial cells[J]. Journal of cellular biochemistry, 2010, 110(4): 857-865.

Li, Z. Y., Zhu, S. S., Chen, X. J., Zhu, J., Chen, Q., Zhang, Y. Q., ... \& Zhang, L. M. (2017). ARID1A suppresses malignant transformation of human pancreatic cells via mediating senescence-associated miR-503/CDKN2A regulatory axis. Biochemical and biophysical research communications, 493(2), 1018-1025.

Liang, H., Cheung, L. W., Li, J., Ju, Z., Yu, S., Stemke-Hale, K., .. \& Guo, W. (2012). Whole-exome sequencing combined with functional genomics reveals novel candidate driver cancer genes in endometrial cancer. Genome research, 22(11), 
2120-2129.

Lickert $\mathrm{H}$, Takeuchi J K, von Both I, et al. Baf60c is essential for function of BAF chromatin remodelling complexes in heart development[J]. Nature, 2004, 432(7013): 107-112.

Liou G Y, Döppler H, Braun U B, et al. Protein kinase D1 drives pancreatic acinar cell reprogramming and progression to intraepithelial neoplasia[J]. Nature communications, 2015, 6(1): 1-11.

Liou G Y, Döppler H, DelGiorno K E, et al. Mutant KRas-induced mitochondrial oxidative stress in acinar cells upregulates EGFR signaling to drive formation of pancreatic precancerous lesions[J]. Cell reports, 2016, 14(10): 2325-2336.

Liou G Y, Döppler H, Necela B, et al. Macrophage-secreted cytokines drive pancreatic acinar-to-ductal metaplasia through NF-kB and MMPs[J]. Journal of Cell Biology, 2013, 202(3): 563-577.

Liu J, Liu S, Gao H, et al. Genome-wide studies reveal the essential and opposite roles of ARID1A in controlling human cardiogenesis and neurogenesis from pluripotent stem cells[J]. Genome biology, 2020, 21(1): 1-27.

Livshits G, Alonso-Curbelo D, Morris IV J P, et al. Arid1a restrains Kras-dependent changes in acinar cell identity[J]. Elife, 2018, 7: e35216.

Logsdon CD, Ji B. Ras activity in acinar cells links chronic pancreatitis and pancreatic cancer. Clin Gastroenterol Hepatol. 2009; 7:S40-S43. [PubMed: 19896097]

Lohr M, Kloppel G, Maisonneuve P, Lowenfels AB, Luttges J. Frequency of K-ras mutations in pancreatic intraductal neoplasias associated with pancreatic ductal adenocarcinoma and chronic pancreatitis: a meta-analysis. Neoplasia 2005;7:1723. [PubMed: 15720814]

Lou, Zhenkun, Katherine Minter-Dykhouse, and Junjie Chen. "BRCA1 participates in DNA decatenation." Nature structural \& molecular biology 12.7 (2005): 589-593.

Luger K, Mäder AW, Richmond RK, Sargent DF, Richmond TJ. Crystal structure of the nucleosome core particle at 2.8 A resolution. Nature 1997;389:251-260. [PubMed: 9305837]

Macian F. NFAT proteins: key regulators of T-cell development and function[J]. Nature 
Reviews Immunology, 2005, 5(6): 472-484.

Macian F. NFAT proteins: key regulators of T-cell development and function[J]. Nature Reviews Immunology, 2005, 5(6): 472-484.

Maitra A, Fukushima N, Takaori K, Hruban RH. Precursors to invasive pancreatic cancer. Adv Anat Pathol 2005;12:81-91. [PubMed: 15731576]

Mammucari C, di Vignano AT, Sharov AA, Neilson J, Havrda MC, Roop DR, Botchkarev VA, Crabtree GR, Dotto GP (2005) Integration of Notch 1 and calcineurin/NFAT signaling pathways in keratinocyte growth and differentiation control. Dev Cell 8: $665-676$

Mamo, A., Cavallone, L., Tuzmen, S., Chabot, C., Ferrario, C., Hassan, S., ... \& Malcolm, K. (2012). An integrated genomic approach identifies ARID1A as a candidate tumorsuppressor gene in breast cancer. Oncogene, 31(16), 2090-2100.

Mancini M, Toker A. NFAT proteins: emerging roles in cancer progression[J]. Nature Reviews Cancer, 2009, 9(11): 810-820.

Marks P A, Richon V M, Breslow R, et al. Histone deacetylase inhibitors as new cancer drugs[J]. Current opinion in oncology, 2001, 13(6): 477-483.

Martens J A, Winston F. Evidence that Swi/Snf directly represses transcription in S. cerevisiae[J]. Genes \& development, 2002, 16(17): 2231-2236.

Martens JA, Wu PY, Winston F (2005) Regulation of an intergenic transcript controls adjacent gene transcription in Saccharomyces cerevisiae. Genes Dev 19(22):26952704.

Mathur R, Alver B H, San Roman A K, et al. ARID1A loss impairs enhancer-mediated gene regulation and drives colon cancer in mice[J]. Nature genetics, 2017, 49(2): 296.

Means A L, Meszoely I M, Suzuki K, et al. Pancreatic epithelial plasticity mediated by acinar cell transdifferentiation and generation of nestin-positive intermediates[J]. Development, 2005, 132(16): 3767-3776.

Mizrahi J D, Surana R, Valle J W, et al. Pancreatic cancer[J]. The Lancet, 2020, 395(10242): 2008-2020. 
Mohammed S, George Van Buren I I, Fisher W E. Pancreatic cancer: advances in treatment[J]. World Journal of Gastroenterology: WJG, 2014, 20(28): 9354.

Mohrmann L, Verrijzer C P. Composition and functional specificity of SWI2/SNF2 class chromatin remodeling complexes[J]. Biochimica et Biophysica Acta (BBA)-Gene Structure and Expression, 2005, 1681(2-3): 59-73.

Moore, M. J., Goldstein, D., Hamm, J., Figer, A., Hecht, J. R., Gallinger, S., ... \& Campos, D.. Erlotinib plus gemcitabine compared with gemcitabine alone in patients with advanced pancreatic cancer: a phase III trial of the National Cancer Institute of Canada Clinical Trials Group. Journal of clinical oncology, 2007, 25(15), 1960-1966.

Morris J P, Wang S C, Hebrok M. KRAS, Hedgehog, Wnt and the twisted developmental biology of pancreatic ductal adenocarcinoma[J]. Nature Reviews Cancer, 2010, 10(10): 683-695.

Morris S A, Baek S, Sung $\mathrm{M} \mathrm{H}$, et al. Overlapping chromatin-remodeling systems collaborate genome wide at dynamic chromatin transitions[J]. Nature structural \& molecular biology, 2014, 21(1): 73.

Nagarajan, S., Rao, S. V., Sutton, J., Cheeseman, D., Dunn, S., Papachristou, E. K., ... \& Chilamakuri, C. S. R. (2020). ARID1A influences HDAC1/BRD4 activity, intrinsic proliferative capacity and breast cancer treatment response. Nature Genetics, 52(2), 187-197.

Nagl Jr N G, Wang X, Patsialou A, et al. Distinct mammalian SWI/SNF chromatin remodeling complexes with opposing roles in cell-cycle control[J]. The EMBO journal, 2007, 26(3): 752-763.

Navas C, Hernández-Porras I, Schuhmacher A J, et al. EGF receptor signaling is essential for k-ras oncogene-driven pancreatic ductal adenocarcinoma[J]. Cancer cell, 2012, 22(3): 318-330.

Nie, Z., Xue, Y., Yang, D., Zhou, S., Deroo, B. J., Archer, T. K., \& Wang, W. (2000). A specificity and targeting subunit of a human SWI/SNF family-related chromatinremodeling complex. Molecular and cellular biology, 20(23), 8879-8888.

Ntranos, Achilles, and Patrizia Casaccia. "Bromodomains: Translating the words of lysine acetylation into myelin injury and repair." Neuroscience letters 625 (2016): 4- 
10.

Ogiwara, H., et al. "Histone acetylation by CBP and p300 at double-strand break sites facilitates SWI/SNF chromatin remodeling and the recruitment of non-homologous end joining factors." Oncogene 30.18 (2011): 2135-2146.

O'Hagan R C, Heyer J. KRAS mouse models: modeling cancer harboring KRAS mutations[J]. Genes \& cancer, 2011, 2(3): 335-343.

Park S J, Gu M J, Lee D S, et al. EGFR expression in pancreatic intraepithelial neoplasia and ductal adenocarcinoma[J]. International journal of clinical and experimental pathology, 2015, 8(7): 8298.

Patel D J, Wang Z. Readout of epigenetic modifications[J]. Annual review of biochemistry, 2013, 82: 81-118.

Phelan M L, Sif S, Narlikar G J, et al. Reconstitution of a core chromatin remodeling complex from SWI/SNF subunits[J]. Molecular cell, 1999, 3(2): 247-253.

Pin C L, Rukstalis J M, Johnson C, et al. The bHLH transcription factor Mist1 is required to maintain exocrine pancreas cell organization and acinar cell identity[J]. The Journal of cell biology, 2001, 155(4): 519-530.

Puri S, Folias A E, Hebrok M. Plasticity and dedifferentiation within the pancreas: development, homeostasis, and disease[J]. Cell stem cell, 2015, 16(1): 18-31.

Pylayeva-Gupta Y, Grabocka E, Bar-Sagi D. RAS oncogenes: weaving a tumorigenic web[J]. Nature Reviews Cancer, 2011, 11(11): 761-774.

Qu Y L, Deng C H, Luo Q, et al. Arid1a regulates insulin sensitivity and lipid metabolism[J]. EBioMedicine, 2019, 42: 481-493.

Rao A, Luo C, Hogan P G. Transcription factors of the NFAT family: regulation and function[J]. Annual review of immunology, 1997, 15(1): 707-747.

Reisman D N, Sciarrotta J, Wang W, et al. Loss of BRG1/BRM in human lung cancer cell lines and primary lung cancers: correlation with poor prognosis[J]. Cancer research, 2003, 63(3): 560-566.

Reisman D, Glaros S, Thompson E A. The SWI/SNF complex and cancer[J]. Oncogene, 2009, 28(14): 1653-1668. 
Reisman, D. N., Sciarrotta, J., Wang, W., Funkhouser, W. K. \& Weissman, B. E. Loss of BRG1/BRM in human lung cancer cell lines and primary lung cancers: correlation with poor prognosis. Cancer Res. 63, 560-566 (2003)

Reisman, D., Glaros, S. \& Thompson, E. The SWI/SNF complex and cancer. Oncogene 28, 1653-1668 (2009). https://doi.org/10.1038/onc.2009.4

Ribeiro-Silva C, Vermeulen W, Lans H. SWI/SNF: Complex complexes in genome stability and cancer. DNA Repair (Amst). 2019 May;77:87-95. doi: 10.1016/j.dnarep.2019.03.007. Epub 2019 Mar 15. PMID: 30897376.

Roberts C W M, Orkin S H. The SWI/SNF complex-chromatin and cancer[J]. Nature Reviews Cancer, 2004, 4(2): 133-142.

Roberts C W M, Leroux M M, Fleming M D, et al. Highly penetrant, rapid tumorigenesis through conditional inversion of the tumor suppressor gene Snf5[J]. Cancer cell, 2002, 2(5): 415-425.

Roberts, C. W., Galusha, S. A., McMenamin, M. E., Fletcher, C. D. \& Orkin, S. H. Haploinsufficiency of Snf5 (integrase interactor 1) predisposes to malignant rhabdoid tumors in mice. Proc. Natl Acad. Sci. USA 97, 13796-13800 (2000).

Romero O A, Sanchez-Cespedes M. The SWI/SNF genetic blockade: effects in cell differentiation, cancer and developmental diseases[J]. Oncogene, 2014, 33(21): 2681-2689.

Rooman I, Real FX. Pancreatic ductal adenocarcinoma and acinar cells: a matter of differentiation and development? Gut. 2012; 61:449-458. [PubMed: 21730103]

Rose S D, Swift G H, Peyton M J, et al. The role of PTF1-P48 in pancreatic acinar gene expression[J]. Journal of Biological Chemistry, 2001, 276(47): 44018-44026.

Rousseau-Merck M F, Versteege I, Legrand I, et al. hSNF5/INI1 inactivation is mainly associated with homozygous deletions and mitotic recombinations in rhabdoid tumors[J]. Cancer Research, 1999, 59(13): 3152-3156.

Roy N, Malik S, Villanueva K E, et al. Brg1 promotes both tumor-suppressive and oncogenic activities at distinct stages of pancreatic cancer formation[J]. Genes \& Development, 2015, 29(6): 658-671. 
Russell R, Perkhofer L, Liebau S, et al. Loss of ATM accelerates pancreatic cancer formation and epithelial-mesenchymal transition[J]. Nature communications, 2015, 6(1): 1-16.

Saha A, Wittmeyer J, Cairns BR. Chromatin remodelling: the industrial revolution of DNA around histones. Nat Rev Mol Cell Biol 2006;7:437-47. [PubMed: 16723979]

Schlessinger J, Lemmon M A. Nuclear signaling by receptor tyrosine kinases: the first robin of spring[J]. Cell, 2006, 127(1): 45-48.

Sen $M$, Wang $X$, Hamdan $F H$, et al. ARID1A facilitates KRAS signaling-regulated enhancer activity in an AP1-dependent manner in colorectal cancer cells[J]. Clinical epigenetics, 2019, 11(1): 1-16.

Serrano M, Lin A W, McCurrach M E, et al. Oncogenic ras provokes premature cell senescence associated with accumulation of p53 and p16INK4a[J]. Cell, 1997, 88(5): 593-602.

Shain A H, Giacomini C P, Matsukuma K, et al. Convergent structural alterations define SWItch/Sucrose NonFermentable (SWI/SNF) chromatin remodeler as a central tumor suppressive complex in pancreatic cancer[J]. Proceedings of the National Academy of Sciences, 2012, 109(5): E252-E259.

Shi G, DiRenzo D, Qu C, et al. Maintenance of acinar cell organization is critical to preventing Kras-induced acinar-ductal metaplasia[J]. Oncogene, 2013, 32(15): 1950-1958.

Shi G, Zhu L, Sun Y, et al. Loss of the acinar-restricted transcription factor Mist1 accelerates Kras-induced pancreatic intraepithelial neoplasia[J]. Gastroenterology, 2009, 136(4): 1368-1378.

Shin, $\mathrm{Ha}$ Youn. "Targeting super-enhancers for disease treatment and diagnosis." Molecules and cells 41.6 (2018): 506.

Shukla V, Vaissière T, Herceg Z. Histone acetylation and chromatin signature in stem cell identity and cancer[J]. Mutation Research/Fundamental and Molecular Mechanisms of Mutagenesis, 2008, 637(1-2): 1-15.

Siegel R, Ma J, Zou Z, et al. Cancer statistics, 2014. CA Cancer J Clin 2014; 64: 9-29. 
Siveke, J.T., Einwächter, H., Sipos, B., Lubeseder-Martellato, C., Klöppel, G.,and Schmid, R.M.. Concomitant pancreatic activation of Kras(G12D)and Tgfa results in cystic papillary neoplasms reminiscent of human IPMN.Cancer Cell, 2007,12, 266279.

Siwak DR, Carey M, Hennessy BT, et al. Targeting the epidermal growth factor receptor in epithelial ovarian cancer: current knowledge and future challenges. J Oncol $2010 ; 2010: 568938$

Slapak E J, Duitman J W, Tekin C, et al. Matrix Metalloproteases in Pancreatic Ductal Adenocarcinoma: Key Drivers of Disease Progression?[J]. Biology, 2020, 9(4): 80.

Somsuan K, Peerapen P, Boonmark W, et al. ARID1A knockdown triggers epithelialmesenchymal transition and carcinogenesis features of renal cells: role in renal cell carcinoma[J]. The FASEB Journal, 2019, 33(11): 12226-12239.

Stanger BZ, Hebrok M. Control of cell identity in pancreas development and regeneration. Gastroenterology. 2013; 144:1170-1179. [PubMed: 23622126]

Stemmer P M, Klee C B. Dual calcium ion regulation of calcineurin by calmodulin and calcineurin B[J]. Biochemistry, 1994, 33(22): 6859-6866.

Storz P. Acinar cell plasticity and development of pancreatic ductal adenocarcinoma[J]. Nature reviews Gastroenterology \& hepatology, 2017, 14(5): 296-304.

Sun X, Wang S C, Wei Y, et al. Arid1a has context-dependent oncogenic and tumor suppressor functions in liver cancer[J]. Cancer cell, 2017, 32(5): 574-589. e6.

Swygert S G, Peterson C L. Chromatin dynamics: interplay between remodeling enzymes and histone modifications[J]. Biochimica et Biophysica Acta (BBA)-Gene Regulatory Mechanisms, 2014, 1839(8): 728-736.

Takeda T, Banno K, Okawa R, et al. ARID1A gene mutation in ovarian and endometrial cancers[J]. Oncology reports, 2016, 35(2): 607-613.

Tobita K, Kijima H, Dowaki S, et al. Epidermal growth factor receptor expression in human pancreatic cancer: Significance for liver metastasis[J]. International journal of molecular medicine, 2003, 11(3): 305-309.

Tolstorukov M Y, Sansam C G, Lu P, et al. Swi/Snf chromatin remodeling/tumor 
suppressor complex establishes nucleosome occupancy at target promoters[J]. Proceedings of the National Academy of Sciences, 2013, 110(25): 10165-10170.

Tripathi A, Dasgupta S, Roy A, et al. Sequential deletions in both arms of chromosome 9 are associated with the development of head and neck squamous cell carcinoma in Indian patients[J]. Journal of experimental and clinical cancer research, 2003, 22(2): 289-298.

Trizzino M, Barbieri E, Petracovici A, et al. The tumor suppressor ARID1A controls global transcription via pausing of RNA polymerase II[J]. Cell reports, 2018, 23(13): 39333945.

Tu Z, Zhuang X, Yao Y G, et al. BRG1 is required for formation of senescence-associated heterochromatin foci induced by oncogenic RAS or BRCA1 loss[J]. Molecular and cellular biology, 2013, 33(9): 1819-1829.

Turner, Bryan M. "Cellular memory and the histone code." Cell 111.3 (2002): 285-291.

Urnov F D, Wolffe A P. Chromatin remodeling and transcriptional activation: the cast (in order of appearance)[J]. Oncogene, 2001, 20(24): 2991-3006.

Utley R T, Côté J, Owen-Hughes T, et al. SWI/SNF stimulates the formation of disparate activator-nucleosome complexes but is partially redundant with cooperative binding[J]. Journal of Biological Chemistry, 1997, 272(19): 12642-12649.

Varela I, Tarpey P, Raine K, et al. Exome sequencing identifies frequent mutation of the SWI/SNF complex gene PBRM1 in renal carcinoma[J]. Nature, 2011, 469(7331): 539-542.

Versteege I, Sévenet N, Lange J, et al. Truncating mutations of hSNF5/INI1 in aggressive paediatric cancer[J]. Nature, 1998, 394(6689): 203-206.

Waerner T, Alacakaptan M, Tamir I, et al. ILEl: a cytokine essential for EMT, tumor formation, and late events in metastasis in epithelial cells[J]. Cancer cell, 2006, 10(3): 227-239.

Wagner M, Greten F R, Weber C K, et al. A murine tumor progression model for pancreatic cancer recapitulating the genetic alterations of the human disease[J]. Genes \& Development, 2001, 15(3): 286-293. 
Wang M T, Fer N, Galeas J, et al. Blockade of leukemia inhibitory factor as a therapeutic approach to KRAS driven pancreatic cancer[J]. Nature communications, 2019, 10(1): 1-10.

Wang S C, Nassour I, Xiao S, et al. SWI/SNF component ARID1A restrains pancreatic neoplasia formation[J]. Gut, 2019, 68(7): 1259-1270.

Wang S, Huang S, Sun Y L. Epithelial-mesenchymal transition in pancreatic cancer: a review[J]. BioMed research international, 2017, 2017.

Wang W, Côté J, Xue Y, et al. Purification and biochemical heterogeneity of the mammalian SWI-SNF complex[J]. The EMBO journal, 1996, 15(19): 5370-5382.

Wang W, Friedland S C, Guo B, et al. ARID1A, a SWI/SNF subunit, is critical to acinar cell homeostasis and regeneration and is a barrier to transformation and epithelialmesenchymal transition in the pancreas[J]. Gut, 2019, 68(7): 1245-1258.

Wang X, Sansam C G, Thom C S, et al. Oncogenesis caused by loss of the SNF5 tumor suppressor is dependent on activity of BRG1, the ATPase of the SWI/SNF chromatin remodeling complex[J]. Cancer research, 2009, 69(20): 8094-8101.

Wang X, Sansam C G, Thom C S, et al. Oncogenesis caused by loss of the SNF5 tumor suppressor is dependent on activity of BRG1, the ATPase of the SWI/SNF chromatin remodeling complex[J]. Cancer research, 2009, 69(20): 8094-8101.

Wang Y, Fischle W, Cheung W, Jacobs S, Khorasanizadeh S, Allis CD. Beyond the double helix: writing and reading the histone code. Novartis Found Symp 2004;259:3-17. discussion 17-21, 163-9. [PubMed: 15171244]

Wang Z, Zhai W, Richardson J A, et al. Polybromo protein BAF180 functions in mammalian cardiac chamber maturation[J]. Genes \& development, 2004, 18(24): 3106-3116.

Wang, K., Kan, J., Yuen, S. T., Shi, S. T., Chu, K. M., Law, S., ... \& Lee, S. P. (2011). Exome sequencing identifies frequent mutation of ARID1A in molecular subtypes of gastric cancer. Nature genetics, 43(12), 1219.

Wangensteen K J, Zhang S, Greenbaum L E, et al. A genetic screen reveals Foxa3 and TNFR1 as key regulators of liver repopulation[J]. Genes \& development, 2015, 29(9): 904-909. 
Watanabe R, Ui A, Kanno S, et al. SWI/SNF factors required for cellular resistance to DNA damage include ARID1A and ARID1B and show interdependent protein stability[J]. Cancer research, 2014, 74(9): 2465-2475.

Wiegand $\mathrm{K} \mathrm{C}$, Shah S P, Al-Agha O M, et al. ARID1A mutations in endometriosisassociated ovarian carcinomas[J]. New England Journal of Medicine, 2010, 363(16): 1532-1543.

Williamson C T, Miller R, Pemberton $\mathrm{H} \mathrm{N}$, et al. ATR inhibitors as a synthetic lethal therapy for tumours deficient in ARID1A[J]. Nature communications, 2016, 7(1): 113.

Wilson B G, Helming K C, Wang X, et al. Residual complexes containing SMARCA2 (BRM) underlie the oncogenic drive of SMARCA4 (BRG1) mutation[J]. Molecular and cellular biology, 2014, 34(6): 1136-1144.

Wilson B G, Helming K C, Wang X, et al. Residual complexes containing SMARCA2 (BRM) underlie the oncogenic drive of SMARCA4 (BRG1) mutation[J]. Molecular and cellular biology, 2014, 34(6): 1136-1144.

Wilson M E, Scheel D, German M S. Gene expression cascades in pancreatic development[J]. Mechanisms of development, 2003, 120(1): 65-80.

Wilson M R, Reske J J, Holladay J, et al. ARID1A and PI3-kinase pathway mutations in the endometrium drive epithelial transdifferentiation and collective invasion[J]. Nature communications, 2019, 10(1): 1-18.

Wilson, M. R., Reske, J. J., Holladay, J., Neupane, S., Ngo, J., Cuthrell, N., ... \& Hostetter, G. (2020). ARID1A Mutations Promote P300-Dependent Endometrial Invasion through Super-Enhancer Hyperacetylation. Cell reports, 33(6), 108366.

Wu $\mathrm{J}$ I. Diverse functions of ATP-dependent chromatin remodeling complexes in development and cancer[J]. Acta biochimica et biophysica Sinica, 2012, 44(1): 5469.

Wu Q, Lian J B, Stein J L, et al. The BRG1 ATPase of human SWI/SNF chromatin remodeling enzymes as a driver of cancer[J]. Epigenomics, 2017, 9(6): 919-931.

Wu R C, Wang T L, Shih I M. The emerging roles of ARID1A in tumor suppression[J]. Cancer biology \& therapy, 2014, 15(6): 655-664. 
Wu Y, Borde M, Heissmeyer V, et al. FOXP3 controls regulatory T cell function through cooperation with NFAT[J]. Cell, 2006, 126(2): 375-387.

Wu Y, Sato F, Yamada T, et al. The BHLH transcription factor DEC1 plays an important role in the epithelial-mesenchymal transition of pancreatic cancer[J]. International journal of oncology, 2012, 41(4): 1337-1346.

Xiong J, Yang $\mathrm{H}$, Luo W, et al. The anti-metastatic effect of 8-MOP on hepatocellular carcinoma is potentiated by the down-regulation of bHLH transcription factor DEC1[J]. Pharmacological research, 2016, 105: 121-133.

Yamanaka, Y., Friess, H., Kobrin, M. S., Buchler, M., Beger, H. G., \& Korc, M. (1993). Coexpression of epidermal growth factor receptor and ligands in human pancreatic cancer is associated with enhanced tumor aggressiveness. Anticancer research, 13(3), 565.

Yarden Y, Sliwkowski MX. Untangling the ErbB signalling network. Nat Rev Mol Cell Biol 2001;2:127-37

Yeo C J, Cameron J L, Lillemoe K D, et al. Pancreaticoduodenectomy with or without distal gastrectomy and extended retroperitoneal lymphadenectomy for periampullary adenocarcinoma, part 2: randomized controlled trial evaluating survival, morbidity, and mortality[J]. Annals of surgery, 2002, 236(3): 355.

Zentner GE, Henikoff S. Regulation of nucleosome dynamics by histone modifications. Nat. Struct. Mol. Biol. 2013; 20:259-266.

Zhang H S, Gavin M, Dahiya A, et al. Exit from G1 and S phase of the cell cycle is regulated by repressor complexes containing HDAC-Rb-hSWI/SNF and RbhSWI/SNF[J]. Cell, 2000, 101(1): 79-89.

Zhang X, Li B, Li W, et al. Transcriptional repression by the BRG1-SWI/SNF complex affects the pluripotency of human embryonic stem cells[J]. Stem cell reports, 2014, 3(3): 460-474.

Zhu L, Shi G, Schmidt C M, et al. Acinar cells contribute to the molecular heterogeneity of pancreatic intraepithelial neoplasia[J]. The American journal of pathology, 2007, 171(1): 263-273.

Zindy F, Eischen C M, Randle D H, et al. Myc signaling via the ARF tumor suppressor Page | 110 
regulates p53-dependent apoptosis and immortalization[J]. Genes \& development, 1998, 12(15): 2424-2433. 
Page | 112 


\section{Acknowledgements}

\section{"Life is not only bread and roses, but also starry sky"- Peiyun Xiong}

Ph.D. is an opportunity which offers people the time and resources to approach the stars one has been chasing for, and in the end, amazingly, one will discover a much broader picture of both nature and himself. Now when I look back into the past few years, many things and moments string up my Ph.D. adventure. During the time I was experiencing them, I might think it was just an ordinary sort of day, now I realize all those things have led up to where and how I am now. Many people have offered me great help and support during the last years, hereby I would like to express my gratitude to all of them!

First of all, I am extremely lucky to have PD Dr. med. Elisabeth Hessmann (Lissy) as my supervisor, it has been so fruitful and joyful to work with her. She provided me with circumspect supervision, encouragement and great support throughout my Ph.D. life. At meanwhile, she also gave me enough understanding and space to think on my own and to pursue my research while silently guiding and ensuring I am on the right track. I have learned a lot from her on how to think logically in project designing and scientific writing. Besides, she is a compass to me with her values and excellences on high efficiency, hard work, being professional and humble and, as a female researcher, balancing work and life.

I sincerely acknowledge the financial support of my Ph.D. study by China Scholarship Council (CSC) from Oct.2016 to Oct. 2020.

On a special note, I would like to express my sincere gratitude to Prof. Dr. Steven A. Johnsen, for his genuine and persistent concern in this project. He and his enthusiasm of science immensely inspired me, especially in the moments when I was self-doubting. This project has experienced a hard time when many literatures in the same field popped up, and he went through that time with us by always being approachable and offering meetings \& discussions with his great brain-storm and wonderful ideas.

Moreover, I very much would like to express appreciation and thanks to Prof. Dr. Bernd Wollnik and Prof. Dr. Argyris Papantonis, for being my thesis advisory committee members and offering helpful suggestions on my project. Besides, my sincere gratitude goes to Prof. Dr. Matthias Dobbelstein, Dr. Nico Posnien and Dr. Ufuk Günesdogan for 
agreeing to be my external examination board members and the great support in arranging my thesis defense.

I appreciate the support received from Dr. Shiv K. Singh for all the fruitful discussions and suggestions he offered to my project, as well as the communications sharing the front-edge scientific news in this field. I specially thank Prof. Dr. med. V. Ellenrieder, for giving me this opportunity to work in this collaborative and enthusiastic department.

I am deeply grateful to the Ph.D. students in Steve's lab - Madhobi Sen, Feda Hamdan for guiding me through high-throughput sequencing methods and specially Xin Wang, who taught me all the useful bioinformatics knowledge, guided me with the programming technique, as well as shared all these memorable discussions in science and out of science. This project would not have reached this level without their help.

Special thanks go to my Ph.D. mates - Shilpa Patil and Lennart Versemann, who are the best working partners and friends ever. They have been always thoughtful, helpful and approachable, regardless of how silly my problem is, listening and providing suggestions to my career- and life- related confusions. They remembered all my likes and dislikes and offered me great joy in everyday life, lab would not have been the same without their company.

Furthermore, I would like to acknowledge the constant technical support from Jessica Spitalieri, Christin Kellner and Kristina Reutlinger, and excellent help of Sercan Mercan and Tobias Kaiser with mice. I am thankful to all the former and present colleagues of our department, for the pleasant and professional working atmosphere you developed in the lab. My special gratitude goes to Waltraut Kopp for always being extremely kind and caring, and providing great help in many aspects of my life. I am deeply grateful to my current and former Ph.D. fellows Umair Latif, Lukas Klein, Geske Schmidt, Nina Pfisterer, Laura Urbach, Marie Hasselluhn, Melanie Patzak and Iswarya Ramu for assisting me with their own expertise and keeping lab life lively with numerous Ph.D. memes. I give special thanks to Mengyu Tu, for all the patience she has in taking care of me. I was and still will be loving to be the first one to try out her new cooking style and share all the big and little things happening in my life.

Last but not least, I whole-heartedly thank my parents and grandmother, who have been my biggest source of strength and courage. Thanks for providing me a wind-proofing 
haven and always offering me unfiltered trust. I would not have ventured to dream and try without their unconditional love and substantial support! 